\title{
Cauchy Problems for Parabolic Equations in Sobolev-Slobodeckii and Hölder Spaces on Uniformly Regular Riemannian Manifolds
}

\author{
Herbert Amann \\ Dedicated to Professor Jan Prüß on the occasion of his retirement
}

\begin{abstract}
In this paper we establish optimal solvability results - maximal regularity theorems - for the Cauchy problem for linear parabolic differential equations of arbitrary order acting on sections of tensor bundles over boundaryless complete Riemannian manifolds $(M, g)$ with bounded geometry. We employ an anisotropic extension of the Fourier multiplier theorem for arbitrary Besov spaces introduced in 4]. This allows for a unified treatment of Sobolev-Slobodeckii and little Hölder spaces. In the flat case $(M, g)=\left(\mathbb{R}^{m},|d x|^{2}\right)$ we recover classical results for Petrowskii-parabolic Cauchy problems.
\end{abstract}

Mathematics Subject Classification (2010). 35K51, 35K52, 58J99.

Keywords. Parabolic initial value problems, noncompact manifolds, maximal regularity, Sobolev-Slobodeckii spaces, Hölder spaces.

\section{Introduction}

It is well-known that parabolic differential equations play an important role in mathematics as well as in more applied sciences, like physics, chemistry, biology, etc. As a rule, sophisticated and complex environments are modeled by (systems of) quasilinear or even fully nonlinear equations. A particularly interesting and important class of nonlinear equations occurring inside mathematics is related to heat flow methods in differential geometry. In such and many other intricate settings even local well-posedness is far from being easily established, if known at all.

In geometry in particular, it is often convenient, or even necessary, to deal with classes of functions possessing relatively high regularity properties. Moreover, it is frequently easier and more appropriate to handle functions 
which are differentiable in the usual point-wise rather than the generalized sense of distributions.

It is a pivotal step in the study of nonlinear parabolic equations to establish maximal regularity results for linear equations. With the help of such tools it is then relatively straightforward to prove the local well-posedness of nonlinear problems by more or less standard linearization techniques.

This paper contains maximal regularity results in Sobolev-Slobodeckii and Hölder spaces of arbitrary order for linear parabolic equations acting on sections of tensor bundles over a vast class of, generally noncompact, Riemannian manifolds. We employ a Fourier-analytic approach which allows for a unified treatment of all these function space settings at one stroke. In order not to overburden this already long paper, we restrict ourselves to manifolds without boundary. Boundary value problems will be treated elsewhere.

For the presentation of our results we need some - rather lengthy preparation on concepts and definitions. We begin by fixing basic syntax.

Let $E, E_{1}, E_{2}$ be Banach spaces over $\mathbb{K}=\mathbb{R}$ or $\mathbb{K}=\mathbb{C}$. Then $\mathcal{L}\left(E_{1}, E_{2}\right)$ is the Banach space of the continuous linear maps from $E_{1}$ into $E_{2}$ endowed with the uniform operator norm, and $\mathcal{L}(E):=\mathcal{L}(E, E)$. By $\mathcal{L}$ is $\left(E_{1}, E_{2}\right)$ we mean the open subset of $\mathcal{L}\left(E_{1}, E_{2}\right)$ of all isomorphisms therein, and $\mathcal{L} \operatorname{aut}(E):=\mathcal{L} \operatorname{is}(E, E)$. We write $(\cdot \mid \cdot)$ and $|\cdot|$ for the Euclidean inner product and norm, respectively, on $\mathbb{K}^{n}$. We identify $a \in \mathcal{L}\left(\mathbb{K}^{m}, \mathbb{K}^{n}\right)$ with its matrix representation $\left[a^{i j}\right] \in \mathbb{K}^{n \times m}$ with respect to the standard bases of $\mathbb{K}^{m}$ and $\mathbb{K}^{n}$, if no confusion seems likely. We endow $\mathbb{K}^{n \times m}$ with the HilbertSchmidt norm which means that the identification $\mathbb{K}^{n \times m}=\mathcal{L}\left(\mathbb{K}^{m}, \mathbb{K}^{n}\right)$ applies.

\section{Tensor Bundles}

Next we collect the needed facts on tensor bundles and refer to [8] or, of course, to [19] for more details and explanations.

Throughout this paper:

- $(M, g)$ is a smooth $m$-dimensional Riemannian manifold with or without boundary.

- $F=\left(F,(\cdot \mid \cdot)_{F}\right)$ is an $n$-dimensional complex inner product space, where $n \in \mathbb{N}$.

If $n=0$, then $F:=\{0\}$ and obvious identifications apply in the following.

As usual, $T M$ denotes the tangent and $T^{*} M$ the cotangent bundle, and

$$
\langle\cdot, \cdot\rangle: T^{*} M \times T M \rightarrow C^{\infty}(M, \mathbb{R})
$$

the (fibre-wise defined) duality pairing. We always suppose

- $\sigma, \tau \in \mathbb{N}$. 
Then $T_{\tau}^{\sigma} M:=T M^{\otimes \sigma} \otimes T^{*} M^{\otimes \tau}$ is the $(\sigma, \tau)$-tensor bundle over $M$ consisting of tensors being contravariant of order $\sigma$ and covariant of order $\tau$. In particular, $T_{0}^{1} M=T M, T_{1}^{0} M=T^{*} M$, and $T_{0}^{0} M=M \times \mathbb{R}$, a trivial line bundle. The covariant metric induced by $g$ on $T^{*} M$ is written $g^{*}$. We endow $T_{\tau}^{\sigma} M$ with the bundle metric $(\cdot \mid \cdot)_{\tau}^{\sigma}:=g^{\otimes \sigma} \otimes g^{* \otimes \tau}$ and the corresponding bundle $\operatorname{norm}|\cdot|_{\tau}^{\sigma}:=\left(u \mapsto \sqrt{(u \mid u)_{\tau}^{\sigma}}\right)$.

The vector bundle of $F$-valued $(\sigma, \tau)$-tensors, $T_{\tau}^{\sigma} M \otimes F$, is defined by

$$
\langle a \otimes f, b\rangle:=f\langle a, b\rangle, \quad a \in T_{\tau}^{\sigma} M, \quad f \in F, \quad b \in T_{\sigma}^{\tau} M .
$$

Here we use the fact that $T_{\tau}^{\sigma} M=\left(T_{\sigma}^{\tau} M\right)^{\prime}$ with respect to the duality pairing $\langle\cdot, \cdot\rangle$ induced by (1.1). We endow $T_{\tau}^{\sigma} M \otimes F$ with the bundle metric

$$
(\cdot \mid \cdot)_{\tau, F}^{\sigma}:=(\cdot \mid \cdot)_{\tau}^{\sigma} \otimes(\cdot \mid \cdot)_{F}=(\cdot \mid \cdot)_{\tau}^{\sigma}(\cdot \mid \cdot)_{F}
$$

and set

$$
\text { - } V=V_{\tau}^{\sigma}=V_{\tau}^{\sigma}(F):=\left(T_{\tau}^{\sigma} M \otimes F,(\cdot \mid \cdot)_{\tau, F}^{\sigma}\right) .
$$

In particular,

$$
V_{0}^{0}(F)=(M \times R) \otimes F \widehat{=} M \times F,
$$

a trivial complex vector bundle of rank $n$ over $M$ if $n \geq 1$, and, if $n=0$, then $V_{0}^{0}(F) \widehat{=} M \times \mathbb{R}$. Here and below, $\widehat{=}$ means 'natural identification'.

Let $W=\left(W,(\cdot \mid \cdot)_{W}\right)$ be any smooth metric vector bundle over $M$. Then $W_{p}$ is its fiber over $p \in M$ and $\Gamma(W)=\Gamma(M, W)$ is the $\mathbb{R}$-vector space of all sections of $W$ (no topology). By $C^{k}(W), \quad k \in \mathbb{N} \cup\{\infty\}$, we mean the $C^{k}(M, \mathbb{R})$-module of all $C^{k}$ sections, and $C^{0}=C$.

We denote by $d v_{g}$ the Riemann-Lebesgue volume measure on $M$. Then $L_{q}(W)$ is, for $1 \leq q \leq \infty$, the Banach space of all (equivalence classes of) $d v_{g}$-measurable sections $u$ of $W$ for which the norm

$$
\|u\|_{q}:=\left(\int_{W}|u|_{W}^{q} d v_{g}\right)^{1 / q}
$$

if $q<\infty$, respectively $\|u\|_{\infty}:=\operatorname{esssup}_{W}|u|_{W}$ if $q=\infty$, is finite.

Assume $\left(x^{1}, \ldots, x^{m}\right)$ is a coordinate system on some open coordinate patch $U$ of $M$. We set

$$
\frac{\partial}{\partial x^{(i)}}:=\frac{\partial}{\partial x^{i_{1}}} \otimes \cdots \otimes \frac{\partial}{\partial x^{i_{r}}}, \quad d x^{(i)}:=d x^{i_{1}} \otimes \cdots \otimes d x^{i_{r}}
$$

for $(i):=\left(i_{1}, \ldots, i_{r}\right) \in \mathbb{J}_{r}:=\{1, \ldots, m\}^{r}$. Then

$$
\frac{\partial}{\partial x^{(i)}} \otimes d x^{(j)}, \quad(i) \in \mathbb{J}_{\sigma}, \quad(j) \in \mathbb{J}_{\tau},
$$

is a coordinate frame for $T_{\tau}^{\sigma} M$ over $U$. We use the summation convention with $(i)$ and $(j)$ running through $\mathbb{J}_{\sigma}$ and $\mathbb{J}_{\tau}$, respectively, Then $a \in V$ has on $U$ the local representation

$$
a \mid U=a_{(j)}^{(i)} \frac{\partial}{\partial x^{(i)}} \otimes d x^{(j)}, \quad a_{(j)}^{(i)} \in F^{U},
$$

where $F$ has to be replaced by $\mathbb{R}$ if $n=0$. 
Given $a \in \Gamma\left(V_{\tau+\sigma}^{\sigma+\tau+\rho}(\mathcal{L}(F))\right)$ and $b \in \Gamma\left(V_{\tau+\rho}^{\sigma}\right)$ with $\rho \in \mathbb{N}$, we define the complet 1 contraction $a \cdot b$ locally by

$$
(a \cdot b) \underset{(j)}{(i)}:=a_{(j)(r)}^{(i)(s)} b_{(s)}^{(r)}
$$

with $(i)$ and $(r)$ running through $\mathbb{J}_{\sigma},(j)$ through $\mathbb{J}_{\tau}$, and $s$ through $\mathbb{J}_{\tau+\rho}$, and where $(i)(s):=\left(i_{1}, \ldots, i_{\sigma}, s_{1}, \ldots, s_{\tau+\rho}\right)$, etc. Then

$$
\Gamma\left(V_{\tau+\sigma}^{\sigma+\tau+\rho}(\mathcal{L}(F))\right) \times \Gamma\left(V_{\tau+\rho}^{\sigma}\right) \rightarrow \Gamma(V), \quad(a, b) \mapsto a \cdot b
$$

is a bilinear vector bundle map which is continuous in the sense that

$$
|a \cdot b|_{V} \leq|a|_{V_{\tau+\sigma}^{\sigma+\tau+\rho}(\mathcal{L}(F))}|b|_{V_{\tau+\rho}^{\sigma}} \cdot
$$

We also need to use the complexification $V_{\mathbb{C}}$ of $V$, defined by

$$
V_{\mathbb{C}}:=\left(T_{\tau}^{\sigma} M \otimes \mathbb{C}\right) \otimes F=T_{\tau}^{\sigma} M \otimes F+i T_{\tau}^{\sigma} M \otimes F,
$$

and continue to write $a \cdot$ for the complexification $(a \bullet)_{\mathbb{C}}$ of $a \bullet$.

For abbreviation, $\mathcal{T} M:=C^{\infty}(T M)$, the $C^{\infty}(M, \mathbb{R})$-module of smooth vector fields on $M$. Then $\nabla=\nabla_{g}$ denotes the Levi-Civita connection on $\mathcal{T} M$. The same symbol is used for its extension over $C^{1}\left(T_{\tau}^{\sigma} M\right)$, considered as an $\mathbb{R}$-linear map

$$
\nabla: C^{1}\left(T_{\tau}^{\sigma} M\right) \rightarrow C\left(T_{\tau+1}^{\sigma} M\right), \quad v \mapsto \nabla v,
$$

where $\nabla=d$, the differential, on $C^{1}(M, \mathbb{R})$ if $\sigma=\tau=0$. For $k \in \mathbb{N}$ we set $\nabla^{k+1}:=\nabla \circ \nabla^{k}$ with $\nabla^{0}:=\mathrm{id}$, and $\nabla(v \otimes f):=\nabla v \otimes f$ for $v \otimes f \in C(V)$. Then $\nabla^{k}$ is an $\mathbb{R}$-linear map

$$
\nabla^{k} \in C^{k}(V) \rightarrow C\left(V_{\tau+k}^{\sigma}\right), \quad u \mapsto \nabla^{k} u .
$$

Note that the $\mathbb{R}$-linearity means 'real differentiation', although $u$ is complexvalued (see (1.3) ).

\section{Normally Elliptic Operators}

Now we are ready to introduce differential operators. We write $\dot{\mathbb{N}}:=\mathbb{N} \backslash\{0\}$ and assume

$$
\text { - } r \in 2 \dot{\mathbb{N}} \text {. }
$$

Let $a_{j} \in C\left(V_{\tau+\sigma}^{\sigma+\tau+j}(\mathcal{L}(F))\right)$ for $0 \leq j \leq r$. We consider the linear differential operator

$$
\mathcal{A}:=\sum_{j=0}^{r} a_{j} \cdot \nabla^{j}
$$

acting on $u \in C^{r}(V)$ by $\left(a_{j} \cdot \nabla^{j}\right) u:=a_{j} \cdot\left(\nabla^{j} u\right)$. With $\mathcal{A}$ we associate its principal symbol $\mathfrak{s} \mathcal{A}$ defined by

$$
\mathfrak{s} \mathcal{A}(\cdot, \xi):=(-1)^{r / 2}\left(a_{r} \cdot \xi^{\otimes r}\right) \cdot, \quad \xi \in \Gamma\left(T^{*} M\right) .
$$

\footnotetext{
1'Complete' means that we contract over a maximal number of indices.
} 
Note that $\mathfrak{s} \mathcal{A}(\cdot, \xi) \in \Gamma\left(\operatorname{End}\left(V_{\mathbb{C}}\right)\right)$, the map $\xi \mapsto \mathfrak{s} \mathcal{A}(\cdot, \xi)$ is $r$-linear, and

$$
|\mathfrak{s} \mathcal{A}(\cdot, \xi)|_{\Gamma\left(\operatorname{End}\left(V_{\mathbb{C}}\right)\right)} \leq\left|a_{r}\right|_{V_{\tau+\sigma}^{\sigma+\tau+r}(\mathcal{L}(F))}\left(|\xi|_{1}^{0}\right)^{r}
$$

for $\xi \in \Gamma\left(T^{*} M\right)$, by (1.4).

We denote by $\sigma(A)$ the spectrum of a linear operator $A$ in a given complex Banach space and write $[\operatorname{Re} z \geq \varepsilon]:=\{z \in \mathbb{C} ; \operatorname{Re} z \geq \varepsilon\}$, etc.

Let $0<\varepsilon \leq 1$. Then $\mathcal{A}$ is uniformly normally $\varepsilon$-elliptic on $(M, g)$ if

$$
\sigma(\mathfrak{s} \mathcal{A}(p, \xi)) \subset[\operatorname{Re} z \geq \varepsilon]
$$

for each $p \in M$ and $\xi \in T_{p}^{*} M$ with $|\xi|_{1}^{0}=1$. It is uniformly normally elliptic if (1.7) holds for some $\varepsilon \in(0,1]$.

Remark 1.1. It is no restriction of generality to assume (1.5). Indeed, if $r$ is odd, then $\sigma\left(\left(a_{r}(p) \cdot(-\xi)^{\otimes r}\right) \cdot\right)=-\sigma\left(\left(a_{r}(p) \cdot \xi^{\otimes r}\right) \cdot\right)$. Thus the spectrum of $\left(a_{r}(p) \cdot \xi^{\otimes r}\right) \cdot$ cannot be contained in one and the same half-space of $\mathbb{C}$ for all $\xi \in T_{p}^{*} M$ with $|\xi|_{1}^{0}=1$.

Remark 1.2. $\mathcal{A}$ is called uniformly strongly $\varepsilon$-elliptic if

$$
\operatorname{Re}(\mathfrak{s} \mathcal{A}(\cdot, \xi) \eta \mid \eta)_{V_{\mathbb{C}}} \geq \varepsilon\left(|\xi|_{1}^{0}\right)^{r}|\eta|_{V_{\mathbb{C}}}^{2}, \quad \xi \in \Gamma\left(T^{*} M\right), \quad \eta \in \Gamma\left(V_{\mathbb{C}}\right) .
$$

It is obvious that this condition implies the uniform normal $\varepsilon$-ellipticity of $\mathcal{A}$.

Remark 1.3. If $\sigma=\tau=n=0$, then $V=M \times \mathbb{R}$ and $\Gamma(V)=\mathbb{R}^{M}$. It follows that $\mathcal{A}$ is uniformly normally $[\varepsilon$-]elliptic iff it is uniformly strongly $[\varepsilon$-]elliptic. In this case, as usual, $\mathcal{A}$ is simply called uniformly [ $\varepsilon$-] elliptic.

Remark 1.4. Assume $(M, g)=\left(\mathbb{R}^{m}, g_{m}\right)$, where $g_{m}$ is the Euclidean metric $\left|d x^{2}\right|:=\left(d x^{1}\right)^{2}+\cdots+\left(d x^{m}\right)^{2}$. If $\sigma=\tau=0$ and $n \geq 1$, then $V=\mathbb{R}^{m} \times F$. We set

$$
D:=-i \partial=-i\left(\partial_{1}, \ldots, \partial_{m}\right)=-i\left(\partial / \partial x^{1}, \ldots, \partial / \partial x^{m}\right)
$$

and use standard multiindex notation. Then we can write $\mathcal{A}$ in the form

$$
\mathcal{A}=\sum_{|\alpha| \leq r} a_{\alpha} D^{\alpha}, \quad a_{\alpha} \in C\left(\mathbb{R}^{m}, \mathcal{L}(F)\right),
$$

and $\mathfrak{s} \mathcal{A}(\cdot, \xi)=\sum_{|\alpha|=r} a_{\alpha} \xi^{\alpha}$ for $\xi \in \mathbb{R}^{m}$. Note that the top-order coefficients are real.

Proof. This follows from $\nabla_{g_{m}}=\partial$, the latter being identified with the Fréchet derivative.

Example 1.5. We denote by

$$
g^{\sharp}: \Gamma\left(T_{\tau+1}^{\sigma} M\right) \rightarrow \Gamma\left(T_{\tau}^{\sigma+1}\right), \quad a \mapsto g^{\sharp} a=: a^{\sharp}
$$

the 'index rising' bundle isomorphism defined by $\left(g^{\sharp} \omega \mid X\right)_{0}^{1}=\langle\omega, X\rangle$ for $\omega$ in $\Gamma\left(T^{*} M\right)$ and $X$ in $\Gamma(T M)$. We write

$$
\mathrm{C}: \Gamma\left(T_{\tau+1}^{\sigma+1} M\right) \rightarrow \Gamma\left(T_{\tau}^{\sigma} M\right), \quad a \mapsto \mathrm{C} a
$$


for the contraction, locally defined by $(\mathrm{C} a)_{(j)}^{(i)}:=a_{(j)(k)}^{(i)(k)}$, with $(i)$ running through $\mathbb{J}_{\sigma},(j)$ through $\mathbb{J}_{\tau}$, and $k$ through $\mathbb{J}_{1}$. Then

$$
\operatorname{div}:=\operatorname{div}_{g}: C^{1}\left(T_{\tau}^{\sigma+1} M\right) \rightarrow C\left(T_{\tau}^{\sigma}\right), \quad a \mapsto \operatorname{div} a:=\mathrm{C}(\nabla a)
$$

is the divergence of $C^{1}$ tensor fields of type $(\sigma+1, \tau)$.

The gradient, $\operatorname{grad} u=\operatorname{grad}_{g} u$, of $u \in C^{1}(M)$ is given by $g^{\sharp} d u$. Thus, if $u \in C^{1}(M)$ and $a \in C^{1}\left(T_{1}^{1} M\right)$,

$$
\operatorname{div}(a \cdot \operatorname{grad} u)=a^{\sharp} \cdot \nabla^{2} u+\operatorname{div}\left(a^{\sharp}\right) \cdot \nabla u .
$$

In local coordinates

$$
\operatorname{div}(a \operatorname{grad} u) \mid U=\frac{1}{\sqrt{g}} \frac{\partial}{\partial x^{i}}\left(\sqrt{g} g^{i j} \frac{\partial u}{\partial x^{j}}\right),
$$

$\left[g^{i j}\right]$ being the inverse of the fundamental matrix, and $\sqrt{g}:=\left(\operatorname{det}\left[g_{i j}\right]\right)^{1 / 2}$. In particular, $\triangle=\triangle_{g}:=$ divgrad is the Laplace-Beltrami operator of $(M, g)$.

Suppose $\sigma=\tau=n=0$. Then

$$
\mathcal{A}:=-\operatorname{div}(a \operatorname{grad} \cdot)
$$

is uniformly $\varepsilon$-elliptic iff

$$
a^{\sharp} \cdot \xi \otimes \xi=\left\langle\xi, a^{\sharp} \xi\right\rangle \geq \varepsilon\left(|\xi|_{1}^{0}\right)^{2}, \quad \xi \in \Gamma\left(T^{*} M\right) .
$$

In local coordinates this means

$$
g^{i k} a_{k}^{j} \xi_{i} \xi_{j} \geq \varepsilon g^{i j} \xi_{i} \xi_{j}, \quad \xi=\xi_{i} d x^{i} .
$$

In particular, $-\triangle$ is uniformly 1-elliptic.

Example 1.6. The covariant Laplacian (or Bochner Laplacian) is defined by $\nabla^{*} \nabla$, where $\nabla^{*}$ is the formal adjoint of $\nabla: C^{\infty}(V) \rightarrow C^{\infty}\left(V_{\tau+1}^{\sigma}\right)$ with respect to the $L_{2}\left(V_{\tau+1}^{\sigma}\right)$ inner product. It is known (e.g., [46, Appen$\operatorname{dix} \mathrm{C}$, Proposition 2.1]) that $\nabla^{*} \nabla=-g^{*} \cdot \nabla^{2}$. Hence $\mathfrak{s} \nabla^{*} \nabla(\cdot, \xi)=\left(|\xi|_{1}^{0}\right)^{2}$ for $\xi \in \Gamma\left(T^{*} M\right)$. Thus $\nabla^{*} \nabla: C^{2}(V) \rightarrow C(V)$ is uniformly normally 1-elliptic.

Example 1.7. For $0 \leq k \leq m$ let $\bigwedge^{k}:=\left(\bigwedge^{k} T^{*} M,(\cdot \mid \cdot)_{k}^{0}\right)$ be the $k$-fold exterior product of $T^{*} M$, considered as a subbundle of $V_{k}^{0}$. Then the Hodge Laplacian

$$
d \delta+\delta d: C^{2}\left(\bigwedge^{k}\right) \rightarrow C\left(\bigwedge^{k}\right)
$$

is uniformly normally 1-elliptic (e.g., [38, Example 10.1.22] and [8]).

\section{Uniformly Regular Riemannian Manifolds}

In order to proceed further we have to assume that $(M, g)$ is a uniformly regular Riemannian manifold. The precise definition of this concept, which has been introduced in [8, is given in Section 9. Here we content ourselves with a list of examples which indicates the extent of this class. If there is no reference given, proofs are found in $[9]$.

Example 1.8. $\left(\mathbb{R}^{m}, g_{m}\right)$ and $\left(\mathbb{R}^{m} \times \mathbb{R}^{+}, g_{m+1}\right)$ are uniformly regular. 
Example 1.9. Every compact manifold is uniformly regular (with respect to any metric $g$ ).

Example 1.10. Products of uniformly regular Riemannian manifolds are uniformly regular.

Example 1.11. Isometric images of uniformly regular Riemannian manifolds are uniformly regular.

Example 1.12. (Manifolds with tame ends) Let $\left(B, g_{B}\right)$ be an $(m-1)$ dimensional compact Riemannian submanifold of $\left(\mathbb{R}^{d}, g_{d}\right), d \geq m$, without boundary. Suppose $0 \leq \alpha \leq 1$. Set

$$
F_{\alpha}(B):=\left\{\left(t, t^{\alpha} y\right) ; t>1, y \in B\right\} \subset \mathbb{R} \times \mathbb{R}^{d}=\mathbb{R}^{d+1} .
$$

Then $F_{0}(B)$ is an infinite cylinder with base $B$, and $F_{1}(B)$ is a (blunt) cone over $B$. We endow $F_{\alpha}(B)$ with the Riemannian metric $g_{F_{\alpha}(B)}$ induced by its embedding into $\left(\mathbb{R}^{d+1}, g_{d+1}\right)$. Assume $M=V_{0} \cup V_{1}$, where $V_{0}$ and $V_{1}$ are open, $V_{0}$ and $V_{0} \cap V_{1}$ are relatively compact, and $\left(V_{1}, g\right)$ is isometric to $\left(F_{\alpha}(B), g_{F_{\alpha}(B)}\right)$. Then $V_{1}$ is a tame end of $M$. Any Riemannian manifold with finitely many pair-wise disjoint tame ends is uniformly regular. In particular, manifolds with cylindrical or 'infinite' conical ends are uniformly regular.

Example 1.13. (Manifolds with cuspidal singularities) Let $(\Omega, \tilde{g})$ be a Riemannian manifold with nonempty compact boundary $\partial \Omega$. Suppose $\beta \geq 1$. Let $\stackrel{\circ}{\Omega}$ be the interior of $\Omega$. Fix $\rho \in C^{\infty}(\stackrel{\Omega}{\Omega},(0,1])$ with $\rho(x)=\left(\operatorname{dist}_{\Omega}(x, \partial \Omega)\right)^{\beta}$ for $x$ in some sufficiently small neighborhood of $\partial \Omega$. Set $(M, g):=\left(\Omega, \tilde{g} / \rho^{2}\right)$. Then $(M, g)$ is uniformly regular.

As an example we see that the Poincaré model of the hyperbolic $m$-space, $\left(\mathbb{B}^{m}, 4 d x^{2} /\left(1-|x|^{2}\right)^{2}\right)$, where $\mathbb{B}^{m}$ is the open unit ball in $\mathbb{R}^{m}$, is a uniformly regular Riemannian manifold.

Example 1.14. If $\partial M=\emptyset$, then $(M, g)$ is uniformly regular iff it has bounded geometry. By this we mean that it is geodesically complete, has a positive injectivity radius, and all covariant derivatives of the curvature tensor are bounded.

Proof. The necessity part is Theorem 4.1 in [9]. The sufficiency statement has been shown by D. Disconzi, Y. Shao, and G. Simonett [20].

Remark 1.15. Under the conditions of Example $1.13,(\Omega, \tilde{g})$ is an instance of a singular manifold as introduced in [8]. If $\mathcal{A}$ is a uniformly normally elliptic differential operator on $(M, g):=\left(\stackrel{\Omega}{\Omega}, \tilde{g} / \rho^{2}\right)$, then, considered as a differential operator on $(\Omega, \tilde{g})$, its coefficients degenerate near the boundary $\partial \Omega$ (cf. [10] for a discussion of this aspect in the case of second order scalar equations). 


\section{Function Spaces}

It has been shown in [8] (also see [7]) that Sobolev-Slobodeckii and Hölder spaces on uniformly regular Riemannian manifolds are well-behaved in the sense that they possess the same embedding, interpolation, and trace properties as in the classical Euclidean case. Moreover, what is most crucial for our purposes, they can be characterized by local coordinates induced by a uniformly regular atlas (see Theorem 9.2 below).

In order to formulate our results on parabolic differential equations we have to introduce these function spaces. Thus we assume throughout that

- $(M, g)$ is a uniformly regular Riemannian manifold

- $1 \leq q \leq \infty$.

We denote by $C_{c}^{\infty}(V)$ the vector space of smooth sections of $V$ with compact support. Furthermore, $(\cdot, \cdot)_{q, \theta}$ is the real, and $(\cdot, \cdot)_{\infty, \theta}^{0}$ the continuous interpolation functor of order $\theta \in(0,1)$ (cf. [3, Section I.2] for a summary of interpolation theory).

For $k \in \mathbb{N}$ we set

$$
\|\cdot\|_{k, q}:=\sum_{j=0}^{k}\left\|\nabla^{j} \cdot\right\|_{L_{q}\left(V_{\tau+j}^{\sigma}\right)} .
$$

Suppose $q<\infty$. Then $W_{q}^{k}(V):=\left(W_{q}^{k}(V),\|\cdot\|_{k, q}\right)$, the Sobolev space of order $k$ (of sections of $V$ ), is the completion of $C_{c}^{\infty}(V)$ in $L_{q}(V)$ with respect to the norm $\|\cdot\|_{k, q}$. Hence $W_{q}^{0}(V)=L_{q}(V)$. If $k<s<k+1$, then

$$
W_{q}^{s}(V):=\left(W_{q}^{k}(V), W_{q}^{k+1}(V)\right)_{q, s-k}
$$

defines the Slobodeckii space of order $s$.

By $B C^{k}(V)$ we mean the closed $\left(\mathbb{R}\right.$-)linear subspace of $C^{k}(V)$ consisting of all $u \in C^{k}(V)$ satisfying $\|u\|_{k, \infty}<\infty$, and $B C:=B C^{0}$. It is a Banach space with the norm $\|\cdot\|_{k, \infty}$. If $k<s<k+1$, then

$$
B C^{s}(V):=\left(B C^{k}(V), B C^{k+1}(V)\right)_{s-k, \infty}
$$

is the Hölder space and

$$
b c^{s}(V):=\left(B C^{k}(V), B C^{k+1}(V)\right)_{s-k, \infty}^{0}
$$

the little Hölder space of order $s$.

Remark 1.16. Suppose $(M, g)=\left(\mathbb{R}^{m}, g_{m}\right)$ and $\sigma=\tau=0$. For $0<\theta<1$ we set

$$
[u]_{\theta, q}:=\left(\int_{\mathbb{R}^{m} \times \mathbb{R}^{m}}\left(\frac{|u(x)-u(y)|_{F}}{|x-y|^{\theta}}\right)^{q} \frac{d(x, y)}{|x-y|^{m}}\right)^{1 / q}, \quad q<\infty,
$$

and

$$
[u]_{\theta, \infty}^{\delta}:=\sup _{\substack{x, y \in \mathbb{R}^{m} \\ 0<|x-y|<\delta}} \frac{|u(x)-u(y)|_{F}}{|x-y|^{\theta}}
$$


where $0<\delta \leq \infty$ and $[\cdot]_{\theta, \infty}:=[\cdot]_{\theta, \infty}^{\infty}$. Then, given $k<s<k+1$ with $k \in \mathbb{N}$,

$$
\|u\|_{s, q}:=\|u\|_{k, q}+\sum_{|\alpha|=k}\left[\partial^{\alpha} u\right]_{s-k, q}
$$

is an equivalent norm for $W_{q}^{s}\left(\mathbb{R}^{m}, F\right)$ if $q<\infty$, and for $B C^{s}\left(\mathbb{R}^{m}, F\right)$ and $b c^{s}\left(\mathbb{R}^{m}, F\right)$ if $q=\infty$. Furthermore, $u \in b c^{s}\left(\mathbb{R}^{m}, F\right)$ iff $u \in B C^{k}\left(\mathbb{R}^{m}, F\right)$ and $\lim _{\delta \rightarrow 0}\left[\partial^{\alpha} u\right]_{s-k, \infty}^{\delta}=0$ for $\alpha \in \mathbb{N}^{m}$ with $|\alpha|=k$. This explains the names 'Slobodeckii' and 'little Hölder' spaces.

It should be observed that definitions (1.10) and (1.11) remain meaningful if $F$ is replaced by any Banach space and $\mathbb{R}^{m}$ by an $m$-dimensional interval.

Suppose $0 \leq s_{0}<s_{1}$. Then

$$
W_{q}^{s_{1}}(V) \stackrel{d}{\hookrightarrow} W_{q}^{s_{0}}(V), \quad q<\infty,
$$

where $\hookrightarrow$ means 'continuous' and $\stackrel{d}{\hookrightarrow}$ 'continuous and dense' injection. Similarly, if $0 \leq s_{0}<s_{1}<s_{2}$ with $s_{1} \notin \mathbb{N}$,

$$
B C^{s_{2}}(V) \stackrel{d}{\hookrightarrow} b c^{s_{1}}(V) \hookrightarrow B C^{s_{1}}(V) \hookrightarrow B C^{s_{0}}(V) .
$$

Consequently,

$$
b c^{s_{1}}(V) \stackrel{d}{\hookrightarrow} b c^{s_{0}}(V), \quad s_{0}, s_{1} \in \mathbb{R}^{+} \backslash \mathbb{N} .
$$

In addition, we need anisotropic spaces on 'time cylinders' over $M$. For this we assume

(i) $0<T<\infty$ and $J=J_{T}:=[0, T]$, or $J=\mathbb{R}^{+}$;

(ii) $1 / \vec{r}:=(1,1 / r)$,

so that $s / \vec{r}=(s, s / r)$ for $s \in \mathbb{R}$. Then we set, for $s \in \mathbb{R}^{+}$,

$$
W_{q}^{s / \vec{r}}(V \times J):=L_{q}\left(J, W_{q}^{s}(V)\right) \cap W_{q}^{s / r}\left(J, L_{q}(V)\right), \quad q<\infty,
$$

and

$$
b c^{s / \vec{r}}(V \times J):=B U C\left(J, b c^{s}(V)\right) \cap b c^{s / r}(J, B C(V)), \quad s \notin \mathbb{N},
$$

where BUC means 'bounded and uniformly continuous'. As mentioned above, these spaces have been investigated in [8], and in the anisotropic case in [7, to which we refer for proofs of (1.12) and (1.13). More precisely, in those papers only $1<q<\infty$ and $n=0$ have been considered. However, it is straightforward to extend those results to the present setting.

Suppose that $q>1$ if $s \in \mathbb{N}$. Then it is shown in [1] that

$$
\begin{aligned}
& u \in W_{q}^{(s+r) / \vec{r}}(V \times J) \text { iff } \nabla^{j} u \in W_{q}^{s / \vec{r}}\left(V_{\tau+j}^{\sigma} \times J\right) \\
& \text { for } 0 \leq j \leq r \text { and } \partial_{t} u \in W_{q}^{s / \vec{r}}(V \times J) .
\end{aligned}
$$

Similarly, if $s \notin \mathbb{N}$,

$$
\begin{aligned}
& u \in b c^{(s+r) / \vec{r}}(V \times J) \text { iff } \nabla^{j} u \in b c^{s / \vec{r}}\left(V_{\tau+j}^{\sigma} \times J\right) \\
& \text { for } 0 \leq j \leq r \text { and } \partial_{t} u \in b c^{s / \vec{r}}(V \times J) .
\end{aligned}
$$


Remark 1.17. For simplicity, we consider $F$-valued tensor bundles only. However, all results of this paper remain valid if $V$ is an arbitrary uniformly regular vector bundle endowed with a uniformly regular metric and a uniformly regular bundle connection (see [7] for definitions). In particular, the tensor bundles $\bigwedge^{k} T^{*} M, \quad 0 \leq k \leq m$, are special instances of this more general setting (cf. 8]). This puts Example 1.7 into perspective.

\section{Parabolic Equations}

We consider initial value problems

$$
\left(\partial_{t}+\mathcal{A}\right) u=f \text { on } M \times J, \quad u(0)=u_{0} \text { on } M .
$$

Here $\mathcal{A}$ is a differential operator of the form (1.6), operating on sections of $V$, but with $t$-dependent coefficients. More precisely, $\mathcal{A}$ is said to be $\bar{s}$-regular, where $\bar{s} \in \mathbb{R}^{+} \backslash \mathbb{N}$, if

$$
a_{j} \in b c^{\bar{s} / \vec{r}}\left(V_{\tau+\sigma}^{\sigma+\tau+j}(\mathcal{L}(F)) \times J\right), \quad 0 \leq j \leq r .
$$

This assumption guarantees the continuity of $\partial+\mathcal{A}$ on anisotropic spaces.

Proposition 1.18. Let $\mathcal{A}$ be $\bar{s}$-regular. Then

$$
\partial_{t}+\mathcal{A} \in \mathcal{L}\left(W_{q}^{(s+r) / \vec{r}}(V \times J), W_{q}^{s / \vec{r}}(V \times J)\right), \quad 0 \leq s<\bar{s},
$$

and

$$
\partial_{t}+\mathcal{A} \in \mathcal{L}\left(b c^{(s+r) / \vec{r}}(V \times J), b c^{s / \vec{r}}(V \times J)\right), \quad 0<s \leq \bar{s}, \quad s \notin \mathbb{N} .
$$

Proof. This is a consequence of the (straightforward extension of the) pointwise multiplier Theorem 9.2 in [8].

Remark 1.19. The $\bar{s}$-regularity assumption has been imposed for simplicity. It is optimal for (1.21), but not for (1.20). Also note that it follows from (1.13) that condition (1.19) in (1.21) can be replaced by

$$
a_{j} \in B C^{\bar{s} / \vec{r}}\left(V_{\tau+\sigma}^{\sigma+\tau+j}(\mathcal{L}(F)) \times J\right), \quad 0 \leq j \leq r,
$$

if $s<\bar{s}$.

Remark 1.20. If $\mathcal{A}$ is autonomous, that is, its coefficients are independent of $t \in J$, then (1.19) reduces to $a_{j} \in b c^{\bar{s}}\left(V_{\tau+\sigma}^{\sigma+\tau+j}(\mathcal{L}(F))\right)$ for $0 \leq j \leq r$.

Remark 1.21. Suppose $\sigma=\tau=0$ and $(M, g)=\left(\mathbb{R}^{m}, g_{m}\right)$. Then, writing $\mathcal{A}$ in the form (1.8), $\bar{s}$-regularity means $a_{\alpha} \in b c^{\bar{s} / \vec{r}}\left(\mathbb{R}^{m} \times J, \mathcal{L}(F)\right)$ for $|\alpha| \leq r$.

Let $\mathcal{A}$ be $\bar{s}$-regular. We write $a_{j}(t)(p):=a_{j}(p, t)$ for $(p, t) \in M \times J$. Then

$$
a_{j}(t) \in b c^{\bar{s}}\left(V_{\tau+\sigma}^{\sigma+\tau+j}(\mathcal{L}(E))\right), \quad t \in J .
$$

Hence

$$
\mathcal{A}(t):=\sum_{j=0}^{r} a_{j}(t) \cdot \nabla^{j}
$$


is well-defined for $t \in J$. The operator (family) $\mathcal{A}$ is uniformly normally [ $\varepsilon$-]elliptic on $M \times J$ if $\mathcal{A}(t)$ has this property uniformly with respect to $t \in J$. Then $\partial_{t}+\mathcal{A}$ is uniformly normally [ $\varepsilon$ - $]$ parabolic.

Remark 1.22. Suppose $\sigma=\tau=0$ and $(M, g)=\left(\mathbb{R}^{m}, g_{m}\right)$. Then $\partial_{t}+\mathcal{A}$ is uniformly normally parabolic iff it is uniformly Petrowskii-parabolic (cf. 34 or 23, for example).

Now we can formulate the main result of this paper. We suppose

(i) $(M, g)$ is a uniformly regular Riemannian manifold without boundary.

(ii) $J=J_{T}$ for some $T>0$.

(iii) $\mathcal{A}$ is $\bar{s}$-regular and uniformly normally elliptic on $M \times J$ of order $r$.

By $\gamma$ we denote the trace operator $u \mapsto u(0)$.

Theorem 1.23. Suppose $k \in \mathbb{N}$ and either

( $\alpha) \quad k r \leq s<k r+1$ and $\bar{s} \geq s$, or

( $\beta) \quad k r+1<s<(k+1) r$ with $s \notin \mathbb{N}$ and $\bar{s}>(k+1) r$.

(i) Assume $\bar{s}>s$ and $1 \leq q<\infty$ with $q>1$ if $s=k r$. Then

$$
\left(\partial_{t}+\mathcal{A}, \gamma\right) \in \mathcal{L} \operatorname{is}\left(W_{q}^{(s+r) / \vec{r}}(V \times J), W_{q}^{s / \vec{r}}(V \times J) \times W_{q}^{s+r(1-1 / q)}(V)\right) .
$$

(ii) Let $s \neq k r$. Then

$$
\left(\partial_{t}+\mathcal{A}, \gamma\right) \in \mathcal{L} \operatorname{is}\left(b c^{(s+r) / \vec{r}}(V \times J), b c^{s / \vec{r}}(V \times J) \times b c^{s+r}(V)\right) .
$$

Remark 1.24. In case (i) the Cauchy problem (1.18) possesses for each $\left(f, u_{0}\right)$ in $W_{q}^{s / \vec{r}}(V \times J) \times W_{q}^{s+r(1-1 / q)}(V)$ a unique solution $u$ belonging to $W_{q}^{(s+r) / \vec{r}}(V \times J)$, and

$$
\|u\|_{W_{q}^{(s+r) / \vec{r}}(V \times J)} \leq c\left(\|f\|_{W_{q}^{s / \vec{r}}(V \times J)}+\left\|u_{0}\right\|_{W_{q}^{s+r(1-1 / q)}(V)}\right) .
$$

Similarly, in case (ii) problem (1.18) has for each

$$
\left(f, u_{0}\right) \in b c^{(s+r) / \vec{r}}(V \times J) \times b c^{s+r}(V)
$$

a unique solution $u \in b c^{(s+r) / \vec{r}}$, and

$$
\|u\|_{b c^{(s+r) / \vec{r}}(V \times J)} \leq c\left(\|f\|_{b c^{(s+r) / \vec{r}}(V \times J)}+\left\|u_{0}\right\|_{b c^{s+r}(V)}\right) .
$$

The proofs below show that $c$ depends on $\varepsilon$, a bound for the $b c^{\bar{s} / \vec{r}}$ norms of the coefficients, and on $T$ only, but not on the individual operators.

Remark 1.25. Suppose $k r<s<k r+1$. Then we can choose $\bar{s}=s$ in part (ii) of the theorem. This regularity assumption is optimal. In contrast, condition $\bar{s}>(k+1) r$ if $k r+1<s<(k+1) r$ is not the best possible one. It stems from the fact that we derive the statements in this case by interpolation (cf. the proof in Section 13). 
Remark 1.26. Suppose $\sigma=\tau=0$ and $(M, g)=\left(\mathbb{R}^{m}, g_{m}\right)$. If $s=k r$, then assertion (i) regains (except for the $\bar{s}$-regularity assumption which we could relax in this situation also) classical results due to V.A. Solonnikov (see 44 and [34, IV.§5 and VII. 9 9]). Our proof is based on Fourier analytic techniques and entirely different from Solonnikov's approach.

Remark 1.27. Assume $\sigma=\tau=0$ and $(M, g)=\left(\mathbb{R}^{m}, g_{m}\right)$. In this case, assertion (ii) is closely related to the Hölder space solvability theory of parabolic equations developed by V.A. Solonnikov (see Theorem VII.10.2 in [34, where even more general parabolic systems are studied).

In the case of scalar parabolic second order equations, Solonnikov's Hölder space results have been partially recovered by A. Lunardi [36, Theorem 5.1.10] using semigroup techniques. Although we could establish a Hölder space theory as well, we prefer to work with little Hölder spaces since the latter enjoy the density properties (1.14).

Remark 1.28. In [27] G. Grubb presented an elaborate extension of the $L_{p}$ theory, $1<p<\infty$, for parabolic (boundary value) problems to manifolds. In fact, she studied pseudodifferential boundary value problems for operators acting on sections of (general) vector bundles over so-called 'admissible manifolds', introduced by her and N.J. Kokholm [29]. These manifolds form a subclass of the family of manifolds with finitely many infinite conical ends (cf. Example 1.12. Thus, for this class and $1<q<\infty$, Theorem 1.23(i) is a very particular special case of Grubb's results (except for her very strong regularity assumptions). The proofs in [27] do, however, not extend to general uniformly regular Riemannian manifolds, since they use in an essential way specific 'admissible' atlases consisting of finitely many charts only (cf. [29, Lemma 1.5]).

Remark 1.29. Let the assumptions of (i) be satisfied. Then it follows from (1.16) and (i) that the homogeneous Cauchy problem

$$
\left(\partial_{t}+\mathcal{A}\right) u=f \text { on } M \times J, \quad u(0)=0
$$

has for each $f \in W_{q}^{s / \vec{r}}(V \times J)$ a unique solution $u$ such that $u, \mathcal{A} u$, and $\partial_{t} u$ belong to $W_{q}^{s / \vec{r}}(V \times J)$.

Similarly, if $s \neq k r$, then (ii) guarantees that (1.22) has for each $f$ in $b c^{s / \vec{r}}(V \times J)$ a unique solution $u$ satisfying $u, \mathcal{A} u, \partial_{t} u \in b c^{s / \vec{r}}(V \times J)$. This shows that Theorem 1.23 provides maximal regularity results.

Let $E_{0}$ and $E_{1}$ be Banach spaces with $E_{1} \stackrel{d}{\hookrightarrow} E_{0}$. Then $\mathcal{H}\left(E_{1}, E_{0}\right)$ denotes the set of all $A \in \mathcal{L}\left(E_{1}, E_{0}\right)$ such that $-A$, considered as a linear operator in $E_{0}$ with domain $E_{1}$, is the infinitesimal generator of a strongly continuous analytic semigroup $\left\{e^{-t A} ; t \geq 0\right\}$ on $E_{0}$, that is, in $\mathcal{L}\left(E_{0}\right)$.

Suppose $s=0$ (so that $1<q<\infty$ ) and let $\mathcal{A}$ be autonomous. It follows from $W_{q}^{0 / \vec{r}}(V \times J) \doteq L_{q}\left(J, L_{q}(V)\right)$ that $\mathcal{A}$ has maximal $L_{q}\left(J, L_{q}(V)\right)$ regularity (cf. 3] or J. Prüss and G. Simonett 39] for explanations). Thus a 
result of G. Dore [21] guarantees that $\mathcal{A}$ belongs to $\mathcal{H}\left(W_{q}^{r}(V), L_{q}^{r}(V)\right)$. The following theorem shows that this is also true if $s>0$.

Theorem 1.30. Let $\mathcal{A}$ be autonomous.

(i) Assume either $s \in r \mathbb{N}$ and $1<q<\infty$, or $s \notin \mathbb{N}$ and $1 \leq q<\infty$. Let $\bar{s}>s$. Then

$$
\mathcal{A} \in \mathcal{H}\left(W_{q}^{s+r}(V), W_{q}^{s}(V)\right) .
$$

(ii) If $s \notin \mathbb{N}$ and $\bar{s} \geq s$, then

$$
\mathcal{A} \in \mathcal{H}\left(b c^{s+r}(V), b c^{s}(V)\right) .
$$

Remark 1.31. Suppose $s=0$ (so that $q>1$ ). Then Theorems $1.23(\mathrm{i})$ and 1.30(i) imply - independently of the Dore result - that $\mathcal{A}$ has maximal $L_{q}\left(J, L_{q}(V)\right)$ regularity. This is already known if $\sigma=\tau=0$ and either $M$ is compact or $(M, g)=\left(\mathbb{R}^{m}, g_{m}\right)$. In fact, it has been shown by H. Amann, M. Hieber, and G. Simonett 12 that then $\mathcal{A}$ has a bounded $H^{\infty}$-calculus, thus, in particular, bounded imaginary powers. Now the assertion is a consequence of the Dore-Venni theorem [22] (see [3, Theorem III.4.10.7] for an exposition). More recently, in the Euclidean space case, maximal $L_{q}\left(J, L_{q}\left(\mathbb{R}^{m}\right)\right)$ regularity has been proved for $1<q<\infty$ - even in infinite-dimensional settings — by R. Denk, M. Hieber, and J. Prüss [17 using rather sophisticated vector-valued harmonic analysis techniques, namely so-called $\mathcal{R}$-boundedness methods (see 39 for a detailed exposition; furthermore, Theorem 6.4.3 therein contains a maximal regularity theorem in higher order Sobolev-Slobodeckii spaces on compact hypersurfaces of $\mathbb{R}^{m}$ without boundary). The approach of our paper is much simpler. If $s \notin \mathbb{N}$, then it can be extended to infinitedimensional settings also. We refrain from doing this here but refer to [1].

Assume $\mathcal{A}$ is an autonomous second order positive semidefinite differential operator with bounded smooth coefficients. Then, by establishing heat kernel bounds and using a result of M. Hieber and J. Prüss 30, A.L. Mazzucato and V. Nistor [37] prove the maximal $L_{p}\left(J, L_{q}(V)\right)$-regularity of $\mathcal{A}$ for $1<p, q<\infty$.

If $s>0$ and $1<q<\infty$, then R. Denk and T. Seger [18] showed that a scalar elliptic operator with constant coefficients generates an analytic semigroup on $W_{q}^{s}\left(\mathbb{R}^{m}\right)$. However, these authors do not establish a maximal regularity result.

Remark 1.32. Suppose $\mathcal{A}$ is autonomous and $0<s \leq \bar{s}$ with $s \notin \mathbb{N}$. Then we can combine Theorem 1.30(ii) with the continuous maximal regularity theory of G. Da Prato and P. Grisvard [14] (see [3, Theorem III.3.4.1]). For this we set

$$
\mathcal{W}_{\infty}^{(s+r, 1)}(V \times J):=C\left(J, b c^{s+r}(V)\right) \cap C^{1}\left(J, b c^{s}(V)\right) .
$$

Then it follows

$$
\left(\partial+\mathcal{A}, \gamma_{0}\right) \in \mathcal{L} \operatorname{is}\left(\mathcal{W}_{\infty}^{(s+r, 1)}(V \times J), C\left(J, b c^{s}(V)\right) \times b c^{s+r}(V)\right) .
$$


Note that, see (1.15),

$$
b c^{s / \vec{r}}(V \times J) \hookrightarrow C\left(J, b c^{s}(V)\right)
$$

and

$$
b c^{(s+r) / \vec{r}}(V \times J)=C\left(J, b c^{s+r}(V)\right) \cap b c^{s / r+1}(J, B C(V)) .
$$

Thus the maximal regularity result obtained from Theorem 1.23 is not comparable to (1.23).

It is the advantage of the anisotropic spaces $b c^{s / \vec{r}}(V \times J)$ over the spaces $\mathcal{W}_{\infty}^{(s+r, 1)}(V \times J)$ that the former enjoy all embedding, interpolation, and trace properties known from the Euclidean case (see [7] and [11]). This is of importance in the study of quasilinear problems. Corresponding results for the $\mathcal{W}$-spaces are, to say the least, not obvious.

In a recent paper, Y. Shao and G. Simonett 43 . established the fact that $\mathcal{A} \in \mathcal{H}\left(b c^{s+2}(V), b c^{s}(V)\right)$ (in the case $n=0$ and $0<s<1$ ), starting with the generation theorem given in the Euclidean case in [5, Theorem 4.2 and Remark 4.6]. Then, using the Da Prato-Grisvard approach — in the extended version of S. Angenent [13] which allows for blow-up at $t=0$ (cf. [3, Theorem III.3.4.1]) — and a regularizing technique of S. Angenent in the modified form of J. Escher, J. Prüss, and G. Simonett 24, the authors establish the local well-posedness and time-analyticity of the Yamabe flow in little Hölder spaces on uniformly regular manifolds.

For further interesting applications of the little Hölder and Sobolev space theory on uniformly regular Riemannian manifolds we refer to Y. Shao 40, 41, and 42], and J. LeCrone and G. Simonett 35].

Remark 1.33. Suppose $\sigma=\tau=n=0$. Then the (generalized) heat operator (1.9) is the negative infinitesimal generator of the 'heat semigroup' $\left\{e^{-t \mathcal{A}} ; t \geq 0\right\}$ on $(M, g)$. More precisely,

$$
\mathcal{A} \in \mathcal{H}\left(W_{q}^{s+2}(M), W_{q}^{s}(M)\right) \text { if } 0 \leq s<\bar{s},
$$

with $q>1$ if $s \in \mathbb{N}$, and

$$
\mathcal{A} \in \mathcal{H}\left(b c^{s+2}(M), b c^{s}(M)\right), \quad 0<s \leq \bar{s}, \quad s \notin \mathbb{N} .
$$

In addition, $\mathcal{A}$ has maximal regularity in the sense of Remark 1.29, The same is true, if $n=0$ and $\sigma$ and $\tau$ are arbitrary, for the covariant Laplacian $\nabla^{*} \nabla$ and for the Hodge Laplacian (with $V$ replaced by $\bigwedge^{k} T^{*} M$ ).

There is an enormous amount of literature concerning heat semigroups on Riemannian manifolds without boundary and bounded geometry. Most of it is an $L_{2}$-theory and deals with kernel estimates and spectral theory (see, for example, E.B. Davies [15] or A. Grigor'yan [26]). Those works rely heavily on curvature bounds which is no issue at all in our approach.

Similarly as for compact manifolds, the cornerstones of the proofs of the above theorems are the corresponding assertions for Euclidean model cases and localizations by means of suitable atlases. In the noncompact setting we cannot use finite atlases but have to deal with infinitely many charts. 
This requires uniform local estimates and a somewhat elaborate technical machinery. Both of these are developed in the following sections.

To allow for a unified approach by Fourier analysis to parabolic and elliptic equations we introduce, in the next section, general weighted spaces on $\mathbb{R}^{d}$ and closed half-spaces thereof. In Section 3 we collect those of their basic properties which we employ in this paper.

The study of anisotropic function spaces and the Fourier analysis therein are considerably facilitated by the use of anisotropic dilations. The latter are introduced in Section 4 and some easy properties are described.

The next section belongs to the heart of the matter. Here we introduce the Fourier multiplier theorems from which we derive our results. In the case of anisotropic Sobolev spaces we rely on the Marcinkiewicz theorem. Anisotropic Slobodeckii and Hölder spaces are particular realizations of Besov spaces. To handle these cases, we introduce an anisotropic extension of the Fourier multiplier theorem established in 4]. Although this extension holds for operatorvalued symbols and arbitrary Banach spaces, we restrict ourselves to the case of matrix-valued symbols. By combining the Fourier multiplier theorem with a lifting property we arrive at simple criteria for Fourier integral operators with (anisotropically) homogeneous symbols to realize bounded linear operators between Sobolev-Slobodeckii, respectively Hölder spaces.

As a first application of these Fourier multiplier theorems we give, in Section 6. a very simple proof for the fact that principal part parabolic operators with constant coefficients define isomorphisms between suitable SobolevSlobodeckii and little Hölder spaces on $\mathbb{R}^{m} \times \mathbb{R}$. It is the advantage of our approach that it handles all these spaces by one and the same technique. In particular, in this Fourier-analytic approach we can deal with all Slobodeckii spaces, including those with integrability index 1, as well as with Hölder spaces. This stands in contrast to the earlier work of other authors. In the Euclidean setting, Solonnikov derived his Hölder space results by carefully estimating heat kernels (also see 25]). However, recently in [45] he has used an anisotropic extension, due to O.A. Ladyzhenskaya [33, of a Fourier multiplier theorem for isotropic Hölder seminorms, given by L. Hörmander in 31 , Theorem 7.9.6], to establish the Hölder continuity of solutions to a number of model problems (also see [16]).

The solvability results of G. Grubb [27] in the Slobodeckii space setting are obtained by first establishing the corresponding results for Bessel potential spaces and then using interpolation. Since the Bessel potential space results are restricted to $L_{q}$ settings with $1<q<\infty$, there is no way to cover the spaces $W_{1}^{s / \vec{r}}$ or Hölder spaces by this method. In addition, interpolation does not lead to optimal regularity conditions for the coefficients.

Using an isotropic setting, we give, along the same lines, in Section 7 a simple proof for Theorem 1.30, provided $\mathcal{A}$ is a principal part operator on $\mathbb{R}^{m}$ 
with constant coefficients. (This result is already contained in [4.) By combining the findings in Sections 6 and 7 we prove in the next section Theorem 1.23 for the constant coefficient model problem on $\mathbb{R}^{m} \times \mathbb{R}^{+}$.

In Section 9 we present the precise definition of uniformly regular Riemannian manifolds and and prove the basic localization Theorem 9.2 The next two sections contain the localization machinery by which we can reduce the proof of Theorem 1.23 and 1.30 to the flat case $(M, g)=\left(\mathbb{R}^{m}, g_{m}\right)$. This is done by constructing a retraction-coretraction pair between our function spaces on $M$ and sequence spaces whose elements take values in the corresponding function spaces on $\mathbb{R}^{m}$. Here we rely on our previous work on function spaces on singular manifolds [7, [8].

In the Euclidean setting in Section 12, we use for the first (and only) time the fact that in the preparatory sections 28 we have dealt with parameterdependent spaces and operators. This is employed to control the lower order terms which, by choosing the parameter sufficiently large, can be considered to be small perturbations of the principal part operators. Thus our use of parameter-dependent spaces is somewhat different from the usual one initiated by M.S. Agranovich and M.I. Vishik [1] and greatly amplified by G. Grubb (see [27, 28] and the references therein).

Finally, in the last section we prove Theorems 1.23 and 1.30 on the basis of the material prepared in the preceding parts.

It should be mentioned that the global strategy applied in this work is more or less well-known, except for the Fourier-analytic treatment of the Hölder space case. Nevertheless, our approach differs in details - even in the Euclidean setting — considerably from those of other authors.

\section{Function Spaces in Euclidean Settings}

We suppose

- $\quad d \in \dot{\mathbb{N}}$ and $\mathbb{X} \in\left\{\mathbb{R}^{d}, \mathbb{H}\right\}$, where $\mathbb{H}=\mathbb{H}^{d}:=\mathbb{R}^{d-1} \times \mathbb{R}^{+}$

and endow $\mathbb{X}$ with the Euclidean metric $g_{d}$. A weight system for $\mathbb{X}$ is a triple $[\ell, \boldsymbol{d}, \boldsymbol{\nu}]$ such that

$$
\left.\begin{array}{l}
\ell \in \dot{\mathbb{N}}, \boldsymbol{d}=\left(d_{1}, \ldots, d_{\ell}\right), \boldsymbol{\nu}=\left(\nu_{1}, \ldots, \nu_{\ell}\right) \in(\dot{\mathbb{N}})^{\ell} \text { with } \\
d_{1}+\cdots+d_{\ell}=d, \text { and } d_{\ell}=1 \text { if } \mathbb{X}=\mathbb{H} .
\end{array}\right\}
$$

We set $\mathbb{X}_{i}:=\mathbb{R}^{d_{i}}$ for $1 \leq i \leq \ell$ with $\mathbb{X}_{\ell}:=\mathbb{R}^{+}$if $\mathbb{X}=\mathbb{H}$. Then $\mathbb{X}_{1} \times \cdots \times \mathbb{X}_{\ell}$ is the $\boldsymbol{d}$-clustering of $\mathbb{X}$. We write

$$
x=\left(x^{1}, \ldots, x^{d}\right)=\left(x_{1}, \ldots, x_{\ell}\right), \quad x_{i}=\left(x_{i}^{1}, \ldots, x_{i}^{d_{i}}\right), \quad 1 \leq i \leq \ell,
$$

according to the interpretation of $x$ as an element of $\mathbb{X}$ or of $\mathbb{X}_{1} \times \cdots \times \mathbb{X}_{d}$.

We call $[\ell, \boldsymbol{d}, \boldsymbol{\nu}]$ reduced weight system if $\ell<d$, and non-reduced otherwise. If $\ell=d$, then $\boldsymbol{d}=\mathbf{1}=(1, \ldots, 1)$. The weight system is $\nu$-homogeneous 
if $\ell=1$. Then $\boldsymbol{d}=(d)$ and $\boldsymbol{\nu}=(\nu)$. In this case we write $[1, d, \nu]$ for $[1, \boldsymbol{d}, \boldsymbol{\nu}]$. In general,

$$
\nu:=\operatorname{LCM}(\boldsymbol{\nu})=\operatorname{LCM}\left(\nu_{1}, \ldots, \nu_{\ell}\right)
$$

the least common multiple of $\nu_{1}, \ldots, \nu_{\ell}$.

With $[\ell, \boldsymbol{d}, \boldsymbol{\nu}]$ we associate its non-reduced version $[d, \mathbf{1}, \boldsymbol{\omega}]$, where

$$
\boldsymbol{\omega}=\boldsymbol{\omega}(\boldsymbol{\nu})=\left(\omega_{1}, \ldots, \omega_{d}\right):=\left(\nu_{1}, \ldots, \nu_{1}, \nu_{2}, \ldots, \nu_{2}, \ldots, \nu_{\ell}, \ldots \nu_{\ell}\right)
$$

with $d_{i}$ copies of $\nu_{i}$. Thus the non-reduced version of $[1, d, \nu]$ equals $[d, \mathbf{1}, \nu \mathbf{1}]$. $\operatorname{Note} \operatorname{LCM}(\boldsymbol{\omega})=\nu$ and

$$
|\boldsymbol{\omega}|:=\omega_{1}+\cdots+\omega_{d}=\boldsymbol{d} \cdot \boldsymbol{\nu}:=d_{1} \nu_{1}+\cdots+d_{\ell} \nu_{\ell} .
$$

Remark 2.1. In this paper only two weight systems will be of importance, namely

(i) trivial, that is, 1-homogeneous weight systems $[1,1,1]$ with $d=m$,

(ii) $r$-parabolic weight systems $[2,(m, 1),(1, r)]$ with $d=m+1$.

Nevertheless, for the sake of a unified presentation it is convenient to consider the general case.

For the following

- we fix a weight system $[\ell, \boldsymbol{d}, \boldsymbol{\nu}]$ for $\mathbb{X}$.

- $E$ is a Banach space.

Given $k \in \nu \mathbb{N}$, we introduce the parameter-dependent norms

$$
\|u\|_{k / \boldsymbol{\nu}, q ; \eta}:=\sum_{\alpha \cdot \boldsymbol{\omega} \leq k} \eta^{k-\alpha \cdot \boldsymbol{\omega}}\left\|\partial^{\alpha} u\right\|_{q}, \quad 1 \leq q \leq \infty
$$

for $\eta>0$. Then parameter-dependent anisotropic Sobolev spaces of order $k / \boldsymbol{\nu}$ over $L_{q}$,

$$
W_{q ; \eta}^{k / \nu}=\left(W_{q}^{k / \nu}(\mathbb{X}, E),\|\cdot\|_{k / \nu, q ; \eta}\right),
$$

are defined for $1 \leq q<\infty$ to be the completion of $\mathcal{S}(\mathbb{X}, E)$ in $L_{q}(\mathbb{X}, E)$ with respect to the norm $\|\cdot\|_{k / \boldsymbol{\nu}, q ; \eta}$. As usual, $\mathcal{S}(\mathbb{X}, E)$ is the Fréchet space of smooth rapidly decreasing $E$-valued functions on $\mathbb{X}$. Then $W_{q ; \eta}^{0 / \nu} \doteq L_{q}$ and $W_{q ; \eta}^{k / \nu} \doteq W_{q}^{k / \nu}:=W_{q ; 1}^{k / \nu}$, where $\doteq$ means: equal except for equivalent norms.

We introduce

$$
B C_{\eta}^{k / \nu}=\left(B C^{k / \nu}(\mathbb{X}, E),\|\cdot\|_{k / \nu, \infty ; \eta}\right),
$$

the Banach space of all $u \in B C(\mathbb{X}, E)$ with $\partial^{\alpha} u \in B C(\mathbb{X}, E)$ for $\alpha \cdot \boldsymbol{\omega} \leq k$, where $B C^{k / \nu}:=B C_{1}^{k / \nu}$. Then

$$
B U C_{\eta}^{k / \nu}=\left(B U C^{k / \nu}(\mathbb{X}, E),\|\cdot\|_{k / \nu, \infty ; \eta}\right)
$$

is the closed linear subspace consisting of all $u$ for which $\partial^{\alpha} u$ is uniformly continuous on $\mathbb{X}$. 
We write $\mathbb{X}_{\hat{\imath}}:=\mathbb{X}_{1} \times \cdots \times \widehat{\mathbb{X}}_{i} \times \cdots \times \mathbb{X}_{\ell}$ for $1 \leq i \leq \ell$, where the hat is the omission symbol, and $\left(x_{i} ; x_{\hat{\imath}}\right)$ stands for $x \in \mathbb{X}$ with $x_{\hat{\imath}} \in \mathbb{X}_{\hat{\imath}}$. Recalling (1.10) and (1.11), we set

$$
\llbracket u \rrbracket_{\theta, q ; i}:= \begin{cases}\left\|x_{\hat{\imath}} \mapsto\left[u\left(\cdot ; x_{\hat{\imath}}\right)\right]_{\theta, q}\right\|_{L_{q}\left(\mathbb{X}_{\hat{\imath}}\right)}, & \text { if } q<\infty, \\ \sup _{x_{\hat{\imath}} \in \mathbb{X}_{\hat{\imath}}}\left[u\left(\cdot ; x_{\hat{\imath}}\right)\right]_{\theta, \infty}, & \text { if } q=\infty .\end{cases}
$$

Suppose $k_{i} \in \nu_{i} \mathbb{N}$ and $k_{i}<s<k_{i}+\nu_{i}$ for $1 \leq i \leq \ell$. Put

$$
\|u\|_{s / \boldsymbol{\nu}, q ; \eta}:=\sum_{i=1}^{\ell}\left(\sum_{j=0}^{k_{i} / \nu_{i}} \eta^{s-j \nu_{i}}\left\|\nabla_{x_{i}}^{j} u\right\|_{q}+\llbracket \nabla_{x_{i}}^{k_{i} / \nu_{i}} u \rrbracket_{\left(s-k_{i}\right) / \nu_{i}, q ; i}\right) .
$$

Let $1 \leq q<\infty$. Then the parameter-dependent anisotropic Slobodeckii space of order $s / \nu$ over $L_{q}$,

$$
W_{q ; \eta}^{s / \nu}=\left(W_{q}^{s / \nu}(\mathbb{X}, E),\|\cdot\|_{s / \nu, q ; \eta}\right),
$$

is the completion of $\mathcal{S}(\mathbb{X}, E)$ in $L_{q}$ with respect to the norm $\|\cdot\|_{s / \boldsymbol{\nu}, q ; \eta}$. The parameter-dependent anisotropic Hölder space of order $s / \nu$ is the Banach space

$$
B U C_{\eta}^{s / \nu}=\left(B U C^{s / \nu}(\mathbb{X}, E),\|\cdot\|_{s / \nu, q ; \eta}\right)
$$

consisting of all $u \in B U C(\mathbb{X}, E)$ such that

$$
\left(x_{i} \mapsto u\left(x_{i} ; \cdot\right)\right) \in B U C^{s / \nu_{i}}\left(\mathbb{X}_{i}, B U C\left(\mathbb{X}_{\hat{\imath}}, E\right)\right)
$$

for $1 \leq i \leq \ell$. (In the Euclidean setting we use the conventional notation $B U C^{t}$ for $B C^{t}$ if $t \in \mathbb{R}^{+} \backslash \mathbb{N}$.) Lastly, the little Hölder space buc ${ }_{\eta}^{s / \nu}$ is the closed linear subspace of $B U C_{\eta}^{s / \nu}$ formed by all $u$ satisfying

$$
\lim _{\delta \rightarrow 0} \sup _{x_{\hat{\imath}} \in \mathbb{X}_{\hat{\imath}}}\left[\nabla_{x_{i}}^{k_{i} / \nu_{i}} u\left(\cdot ; x_{\hat{\imath}}\right)\right]_{\left(s-k_{i}\right) / \nu_{i}, \infty}^{\delta}=0, \quad 1 \leq i \leq \ell .
$$

\section{Basic Properties}

In this section we collect the fundamental facts about the spaces introduced above which are needed in what follows. We do not give proofs but refer to [1] for a detailed exposition, even in vector-valued settings. (Also see [6] for a preliminary account which, however, does not include Hölder spaces).

Henceforth, we denote by $c, c_{0}, c_{1}, \ldots$ constants $\geq 1$ which may depend in an increasing way on nonnegative parameters $\alpha, \beta, \ldots$, whereupon we write $c(\alpha, \beta, \ldots)$ etc. These constants may vary from occurrence to occurrence but are always independent of the free variables in a given setting.

Let $f$ and $g$ be nonnegative functions on some set $S$. Then $f \sim g$ means

$$
g / c \leq f \leq c g .
$$

Suppose $f_{\eta}, g_{\eta}: S \rightarrow \mathbb{R}^{+}$for $\eta>0$. Then we write $f_{\eta} \underset{\eta}{g_{\eta}}$ if $f_{\eta} \sim g_{\eta}$ holds $\eta$-uniformly, that is, the constant $c$ in (3.1) is independent of $\eta>0$. Let $X_{\eta}^{(i)}$ 
be normed vector spaces with norm $\|\cdot\|_{\eta}^{(i)}$ for $\eta>0$. Then $X_{\eta}^{(1)} \doteq X_{\eta}^{(2)}$ iff $\|\cdot\|_{\eta}^{(1)} \underset{\eta}{ }\|\cdot\|_{\eta}^{(2)}$. Suppose $a_{\eta} \in \mathcal{L}\left(X_{\eta}^{(1)}, X_{\eta}^{(2)}\right)$ for $\eta>0$. Then we say: $a_{\eta}$ belongs to $\mathcal{L}\left(X_{\eta}^{(1)}, X_{\eta}^{(2)}\right) \eta$-uniformly, if the norm of $a_{\eta}$ can be bounded independently of $\eta>0$. If, in addition, $a_{\eta}^{-1} \in \mathcal{L}\left(X_{\eta}^{(2)}, X_{\eta}^{(1)}\right) \eta$-uniformly, then $a_{\eta} \in \mathcal{L}$ is $\left(X_{\eta}^{(1)}, X_{\eta}^{(2)}\right) \eta$-uniformly.

To avoid lengthy repetitions, we call $(s, q) \nu$-admissible, if either $s \in \nu \mathbb{N}$ and $1<q<\infty$, or $s \notin \mathbb{N}$ and $1 \leq q \leq \infty$.

Throughout this section

(i) $(s, q)$ is $\nu$-admissible.

(ii) $E, E_{0}, E_{1}, \ldots$ are finite-dimensional complex Banach spaces.

Then we set

$$
\mathfrak{F}_{q ; \eta}^{s / \nu}=\mathfrak{F}_{q ; \eta}^{s / \nu}(\mathbb{X}, E):= \begin{cases}W_{q ; \eta}^{s / \nu}, & \text { if } q<\infty, \\ b u c_{\eta}^{s / \nu}, & \text { if } q=\infty,\end{cases}
$$

for $\eta>0$. We omit $\eta$ if it equals 1 and write $s$ for $s / \nu$ if the weight system is trivial. Thus $\mathfrak{F}_{q}^{s}$ is a standard isotropic Sobolev-Slobodeckii space if $q<\infty$ and an isotropic little Hölder space if $q=\infty$. Observe that $\mathfrak{F}_{q ; \eta}^{s / \nu} \doteq \mathfrak{F}_{q}^{s / \nu}$ (but not $\eta$-uniformly!).

\section{Theorem 3.1.}

(i) Assume $0 \leq s_{0}<s_{1}$ and $\left(s_{i}, q\right)$ are $\nu$-admissible. Then $\mathfrak{F}_{q}^{s_{1} / \nu} \stackrel{d}{\hookrightarrow} \mathfrak{F}_{q}^{s_{0} / \nu}$ and

$$
\|\cdot\|_{s_{0} / \nu, q ; \eta} \leq c \eta^{s_{0}-s_{1}}\|\cdot\|_{s_{1} / \nu, q ; \eta}, \quad \eta>0 .
$$

(ii) If $\alpha \in \mathbb{N}^{d}$, then $\partial^{\alpha} \in \mathcal{L}\left(\mathfrak{F}_{q ; \eta}^{(s+\alpha \cdot \boldsymbol{\omega}) / \nu}, \mathfrak{F}_{q ; \eta}^{s / \nu}\right) \eta$-uniformly.

The spaces $\mathfrak{F}_{q}^{s / \nu}$ enjoy an important intersection space characterization. For this $\mathbb{X}=\mathbb{X}^{\bullet} \times \mathbb{X}_{\ell}$ with $\mathbb{X}^{\bullet}=\mathbb{X}_{1} \times \cdots \times \mathbb{X}_{\ell-1}=\mathbb{R}^{d-d_{\ell}}$ and $\boldsymbol{\nu}=\left(\boldsymbol{\nu}^{\bullet}, \nu_{\ell}\right)$.

Theorem 3.2. If $q<\infty$, then

$$
\begin{aligned}
\mathfrak{F}_{q ; \eta}^{s / \nu} & =W_{q ; \eta}^{s / \nu}\left(\mathbb{X}^{\bullet} \times \mathbb{X}_{\ell}, E\right) \\
& \doteq L_{q}\left(\mathbb{X}_{\ell}, W_{q ; \eta}^{s / \nu}\left(\mathbb{X}^{\bullet}, E\right)\right) \cap W_{q ; \eta}^{s / \nu_{\ell}}\left(\mathbb{X}_{\ell}, L_{q}\left(\mathbb{X}^{\bullet}, E\right)\right)
\end{aligned}
$$

Suppose $q=\infty$. Then

$$
\begin{aligned}
\mathfrak{F}_{\infty ; \eta}^{s / \nu} & =b u c_{\eta}^{s / \nu}\left(\mathbb{X}^{\bullet} \times \mathbb{X}_{\ell}, E\right) \\
& \doteq B U C\left(\mathbb{X}_{\ell}, b u c_{\eta}^{s / \nu}\left(\mathbb{X}^{\bullet}, E\right)\right) \cap b u c_{\eta}^{s / \nu_{\ell}}\left(\mathbb{X}_{\ell}, B U C\left(\mathbb{X}^{\bullet}, E\right)\right)
\end{aligned}
$$

The next theorem concerns point-wise multiplications. For Banach spaces $X_{0}, X_{1}$, and $X_{2}$ we denote by $\mathcal{L}\left(X_{0}, X_{1} ; X_{2}\right)$ the Banach space of all continuous bilinear maps $\beta: X_{0} \times X_{1} \rightarrow X_{2}$. If $\beta \in \mathcal{L}\left(E_{0}, E_{1} ; E_{2}\right)$, then we write $\mathrm{m}_{\beta}$ for its point-wise extension. 
Theorem 3.3. Suppose $s \leq s_{0}$ with $s_{0} \notin \mathbb{N}$ and $s<s_{0}$ if $q<\infty$. Let $\beta$ belong to $\mathcal{L}\left(E_{0}, E_{1} ; E_{2}\right)$. Then

$$
\mathrm{m}_{\beta} \in \mathcal{L}\left(b u c^{s_{0} / \nu}\left(\mathbb{X}, E_{0}\right), \mathfrak{F}_{q ; \eta}^{s / \nu}\left(\mathbb{X}, E_{1}\right) ; \mathfrak{F}_{q ; \eta}^{s / \nu}\left(\mathbb{X}, E_{2}\right)\right) \quad \eta \text {-uniformly. }
$$

If $s \in \nu \mathbb{N}$, then

$$
\mathrm{m}_{\beta} \in \mathcal{L}\left(B U C^{s / \nu}\left(\mathbb{X}, E_{0}\right), \mathfrak{F}_{q ; \eta}^{s / \nu}\left(\mathbb{X}, E_{1}\right) ; \mathfrak{F}_{q ; \eta}^{s / \nu}\left(\mathbb{X}, E_{2}\right)\right) \quad \eta \text {-uniformly. }
$$

In either case, the map $\beta \mapsto \mathrm{m}_{\beta}$ is linear and continuous.

For the the next theorem we recall that $\left(X_{0}, X_{1}\right)_{\theta, q}=\left(X_{0}, X_{1}\right)_{\theta, q}^{0}$, if $X_{0}$ and $X_{1}$ are Banach spaces with $X_{1} \stackrel{d}{\hookrightarrow} X_{0}$ and $q<\infty$.

Theorem 3.4. Let $\left(s_{0}, q\right),\left(s_{1}, q\right)$, and $\left(s_{\theta}, q\right)$ be $\nu$-admissible with $s_{0}<s_{1}$ and $s_{\theta}:=(1-\theta) s_{0}+\theta s_{1}$. Then $\left(\mathfrak{F}_{q ; \eta}^{s_{0} / \nu}, \mathfrak{F}_{q ; \eta}^{s_{1} / \nu}\right)_{\theta, q}^{0} \doteq \mathfrak{\overline { F }}_{q ; \eta}^{s_{\theta} / \nu}$.

A retraction from $X_{0}$ onto $X_{1}$ is a continuous linear map $r: X_{0} \rightarrow X_{1}$ possessing a continuous right inverse $r^{c}$, a coretraction. Any such pair $\left(r, r^{c}\right)$ is said to be an r-e pair for $\left(X_{0}, X_{1}\right)$ (e stands for 'extension').

We identify $\partial \mathbb{H}=\mathbb{X}^{\bullet} \times\{0\}$ naturally with $\mathbb{X}^{\bullet}=\mathbb{R}^{d-1}$ if convenient. Then the trace operator of order $k$ is the map $\gamma^{k}:=\left(u \mapsto \partial_{\ell}^{k} u(0)\right)$ for $k \in \mathbb{N}$, defined for sufficiently smooth functions $u: \mathbb{H} \rightarrow E$. Thus $\gamma=\gamma^{0}$.

Theorem 3.5. Suppose $s>\nu_{\ell}(k+1 / q)$ and $s \notin \mathbb{N}+\nu_{\ell} / q$. Then the trace map $\vec{\gamma}^{k}:=\left(\gamma^{0}, \gamma^{1}, \ldots, \gamma^{k}\right)$ is an $\eta$-uniform retraction

$$
\text { from } \mathfrak{F}_{q ; \eta}^{s / \vec{\nu}}(\mathbb{H}, E) \text { onto } \prod_{j=0}^{k} \mathfrak{F}_{q ; \eta}^{\left(s-\nu_{\ell}(j+1 / q)\right) / \nu}\left(\mathbb{X}^{\bullet}, E\right) \text {. }
$$

It possesses an $\eta$-uniform coretraction.

It follows from Theorem 3.2 that $\mathfrak{F}_{q}^{s / \nu}\left(\mathbb{R}^{d}, E\right) \hookrightarrow L_{q}\left(\mathbb{R}, \mathfrak{F}_{q}^{s / \nu^{\prime}}\left(\mathbb{R}^{d-1}, E\right)\right)$. Hence

$$
{ }_{0} \mathfrak{F}_{q}^{s / \nu}:=\left\{u \in \mathfrak{F}_{q}^{s / \nu}\left(\mathbb{R}^{d}, E\right) ; u(t)=0 \text { a.a. } t<0\right\}
$$

is a well-defined linear subspace of $L_{q}\left(\mathbb{R}, \mathfrak{F}_{q}^{s / \dot{\nu}}\left(\mathbb{R}^{d-1}, E\right)\right)$.

Suppose $k \in \mathbb{N}$ and

$$
\nu_{\ell}(k+1 / q)<s<\nu_{\ell}(k+1+1 / q), \quad s \notin(\mathbb{N}+1 / q) \cup\left(\mathbb{N}+\nu_{\ell} / q\right) .
$$

It is a consequence of this trace theorem that

$$
\mathfrak{F}_{q}^{s / \nu}(\stackrel{\mathbb{H}}{1}, E):=\left\{u \in \mathfrak{F}_{q}^{s / \nu}(\mathbb{H}, E) ; \vec{\gamma}^{k} u=0\right\}
$$

is a closed linear subspace of $\mathfrak{F}_{q}^{s / \nu}(\mathbb{H}, E)$. The next theorem shows that we can extend the elements of $\mathfrak{F}_{q}^{s / \nu}(\mathbb{H}, E)$ and $\mathfrak{F}_{q}^{s / \nu}(\stackrel{\circ}{\mathbb{H}}, E)$ over $\mathbb{R}^{d}$ preserving their regularity. We denote by $\mathfrak{R}$ the operator of point-wise restriction from $\mathbb{R}^{d}$ onto $\mathbb{H}$, and $\stackrel{\mathfrak{E}}{\mathrm{E}}$ is the operator of extension by zero from $\mathbb{H}$ over $\mathbb{R}^{d}$. 


\section{Theorem 3.6.}

(i) $\mathfrak{R} \in \mathcal{L}\left(\mathfrak{F}_{q ; \eta}^{s / \nu}\left(\mathbb{R}^{d}, E\right), \mathfrak{F}_{q ; \eta}^{s / \nu}(\mathbb{H}, E)\right) \eta$-uniformly and there exists a universal $\eta$-uniform coretraction $\mathfrak{E}$ for it. Moreover, $\mathfrak{R}$ commutes with $\partial^{\alpha}$ for $\alpha \cdot \boldsymbol{\omega} \leq s$.

(ii) Assume (3.2) applies. There exists $\stackrel{\Re}{\mathfrak{R}}$ such that $(\stackrel{\circ}{\mathfrak{R}}, \stackrel{\circ}{\mathfrak{E}})$ is an $\eta$-uniform r-e pair for $\left(\mathfrak{F}_{q ; \eta}^{s / \nu}\left(\mathbb{R}^{d}, E\right), \mathfrak{F}_{q ; \eta}^{s / \nu}(\stackrel{\mathbb{H}}{1}, E)\right)$ and such that the restriction of $\mathfrak{R}$ to $\operatorname{im}(\stackrel{\mathfrak{E}}{\mathfrak{E}})$ equals $\mathfrak{R} \mid \operatorname{im}(\stackrel{\mathfrak{E}}{)})$.

(iii) Suppose $0 \leq s<\nu_{\ell} / q$. Then $(\mathfrak{R}$, 主) is an $\eta$-uniform $r$-e pair for $\left(\mathfrak{F}_{q ; \eta}^{s / \nu}\left(\mathbb{R}^{d}, E\right), \mathfrak{F}_{q ; \eta}^{s / \nu}(\mathbb{H}, E)\right)$.

\section{Corollary 3.7.}

(i) Let either (3.2) be satisfied or $0 \leq s<\nu_{\ell} / q$. Then ${ }_{0} \mathfrak{F}_{q}^{s / \nu}$ is a closed linear subspace of $\mathfrak{F}_{q}^{s / \nu}\left(\mathbb{R}^{d}, E\right)$.

(ii) If (3.2) applies, then $\stackrel{\mathfrak{E}}{\in} \in \mathcal{L}\left(\mathfrak{F}_{q ; \eta}^{s / \nu}(\stackrel{\mathbb{H}}{1}, E),{ }_{0} \mathfrak{F}_{q ; \eta}^{s / \nu}\right) \eta$-uniformly.

(iii) Assume $0 \leq s<\nu_{\ell} / q$. Then $\stackrel{\mathfrak{E}}{\in} \mathcal{L}\left(\mathfrak{F}_{q ; \eta}^{s / \nu}(\mathbb{H}, E)\right.$, $\left.{ }_{0} \mathfrak{F}_{q ; \eta}^{s / \nu}\right) \eta$-uniformly.

The universality of $\mathfrak{E}$ means that it has a representation which is independent of $s, q$, and $\eta$.

It is of fundamental importance for what follows that all estimates contained implicitly or explicitly in the preceding theorems hold $\eta$-uniformly.

\section{Anisotropic Dilations}

Henceforth, $\mathbf{Z}:=\mathbb{R}^{d} \times \mathbb{R}^{+}$. Its general point is written as $\zeta=(\xi, \eta)$ with

$$
\xi=\left(\xi_{1}, \ldots, \xi_{\ell}\right) \in \mathbb{R}^{d_{1}} \times \cdots \times \mathbb{R}^{d_{\ell}}=\mathbb{R}^{d} .
$$

We equip $\mathbf{Z}$ with the $\nu$-augmented weight system

$$
[\ell+1,(\boldsymbol{d}, 1),(\boldsymbol{\nu}, \nu)]
$$

that is, we assign the weight $\nu$ to the variable $\eta$. Then

$$
t \cdot \zeta:=\left(t^{\nu_{1}} \xi_{1}, \ldots, t^{\nu_{\ell}} \xi_{\ell}, t^{\nu} \eta\right), \quad t>0, \quad \zeta \in \mathbf{Z},
$$

is the anisotropic dilation on $\mathrm{Z}$ associated with (4.1).

Let $X$ be a Banach space and $\dot{Z}:=Z \backslash\{0\}$. Given $u \in C(\dot{Z}, X)$, we set $\sigma_{t} u(\zeta):=u(t \cdot \zeta)$ for $t>0$ and $\zeta \in \dot{Z}$. Then $u$ is positively $z$-homogeneous (with respect to (4.1)), where $z \in \mathbb{C}$, if $\sigma_{t} u=t^{z} u$ for $t>0$.

The natural quasinorm, $\Lambda: Z \rightarrow \mathbb{R}^{+}$, on $\mathbf{Z}$ (with respect to (4.1)) is defined by

$$
\Lambda(\zeta):=\left(\sum_{i=1}^{\ell}\left|\xi_{i}\right|^{2 \nu / \nu_{i}}+\eta^{2}\right)^{1 / 2 \nu}, \quad \zeta \in \mathrm{Z} .
$$

It is positively 1-homogeneous. Moreover,

$$
r_{\Lambda}: \dot{Z} \rightarrow[\Lambda=1], \quad \zeta \mapsto \Lambda^{-1}(\zeta) \cdot \zeta
$$


is the $\Lambda$-retraction onto $[\Lambda=1]:=\{\zeta \in Z ; \Lambda(\zeta)=1\}$. It is a continuous (topological) retraction, since $\Lambda\left(r_{\Lambda}(\zeta)\right)=\Lambda^{-1}(\zeta) \Lambda(\zeta)=1$.

Assume $z \in \mathbb{C}$ and $a \in C(\dot{Z}, X)$ is positively $z$-homogeneous. If $\alpha \in \mathbb{N}^{d}$ and $\partial_{\xi}^{\alpha} a \in C(\dot{\mathbf{Z}}, X)$, then $\partial_{\xi}^{\alpha}$ is positively $(z-\alpha \cdot \boldsymbol{\omega})$-homogeneous,

$$
\partial_{\xi}^{\alpha} a=\Lambda^{z-\alpha \cdot \omega}\left(\partial_{\xi}^{\alpha} a\right) \circ r_{\Lambda}
$$

and

$$
\left|\partial_{\xi}^{\alpha} a\right|_{X} \leq \Lambda^{\operatorname{Re} z-\alpha \cdot \boldsymbol{\omega}}\left\|\left(\partial_{\xi}^{\alpha} a\right) \circ r_{\Lambda}\right\|_{\infty}
$$

(cf. [6, Lemma 1.2.1]).

By $\mathcal{H}_{z}(\mathrm{Z}, E)$ we denote the vector space of all positively $z$-homogeneous $a \in C(\dot{\mathbf{Z}}, E)$ such that $\partial_{\xi}^{\alpha} a \in C(\dot{\mathbf{Z}}, E)$ for $\alpha \in \mathbb{N}^{d}$ with $\alpha \cdot \boldsymbol{\omega} \leq 2|\boldsymbol{\omega}|$. It is a Banach space with the norm

$$
\|a\|_{\mathcal{H}_{z}}:=\max _{\alpha \cdot \boldsymbol{\omega} \leq 2|\boldsymbol{\omega}|}\left\|\left(\partial_{\xi}^{\alpha} a\right) \circ r_{\Lambda}\right\|_{\infty} .
$$

It is easily verified that

$$
\Lambda^{z} \in \mathcal{H}_{z}(\mathrm{Z}) \text {. }
$$

Let $\beta \in \mathcal{L}\left(E_{0}, E_{1} ; E_{2}\right)$ and $z_{0}, z_{1} \in \mathbb{C}$. Using Leibniz' rule, we get

$$
\mathrm{m}_{\beta} \in \mathcal{L}\left(\mathcal{H}_{z_{0}}\left(\mathrm{Z}, E_{0}\right), \mathcal{H}_{z_{1}}\left(\mathrm{Z}, E_{1}\right) ; \mathcal{H}_{z_{0}+z_{1}}\left(\mathrm{Z}, E_{2}\right)\right)
$$

and the map $\beta \mapsto \mathrm{m}_{\beta}$ is linear and continuous. If $a \in \mathcal{H}_{z}\left(\mathrm{Z}, \mathcal{L} \operatorname{is}\left(E_{0}, E_{1}\right)\right)$, then

$$
a^{-1}:=\left(\zeta \mapsto a(\zeta)^{-1}\right) \in \mathcal{H}_{-z}\left(\mathrm{Z}, \mathcal{L} \operatorname{is}\left(E_{1}, E_{0}\right)\right)
$$

and

$$
\left\|a^{-1}\right\|_{\mathcal{H}_{-z}} \leq c\left(\|a\|_{\mathcal{H}_{z}},\left\|a^{-1} \circ r_{\Lambda}\right\|_{\infty}\right)
$$

(cf. Lemmas 1.4.1 and 1.4.3 in [6]).

Given $a: \dot{Z} \rightarrow X$, we set $a_{\eta}:=a(\cdot, \eta): \mathbb{R}^{d} \rightarrow X$ for $\eta>0$. The linear subspace of $C\left(\mathbb{R}^{d}, E\right)$ of all $a$ satisfying $\partial^{\alpha} a \in C\left(\mathbb{R}^{d}, E\right)$ for $\alpha \cdot \boldsymbol{\omega} \leq 2|\boldsymbol{\omega}|$, endowed with the norm

$$
\|a\|_{\mathcal{M}_{\eta}}:=\max _{\alpha \cdot \boldsymbol{\omega} \leq 2|\boldsymbol{\omega}|}\left\|\Lambda_{\eta}^{\alpha \cdot \boldsymbol{\omega}} \partial^{\alpha} a\right\|_{\infty}<\infty,
$$

is denoted by $\mathcal{M}_{\eta}(E)=\mathcal{M}_{\eta}\left(\mathbb{R}^{d}, E\right)$. It is a Banach space. As a consequence of (4.2) and (4.3) we obtain

$$
\left(a \rightarrow a_{\eta}\right) \in \mathcal{L}\left(\mathcal{H}_{0}(\mathrm{Z}, E), \mathcal{M}_{\eta}(E)\right) \quad \eta \text {-uniformly } .
$$

Similarly as above, if $\beta \in \mathcal{L}\left(E_{0}, E_{1} ; E_{2}\right)$, then

$$
\mathrm{m}_{\beta} \in \mathcal{L}\left(\mathcal{M}_{\eta}\left(E_{0}\right), \mathcal{M}_{\eta}\left(E_{1}\right) ; \mathcal{M}_{\eta}\left(E_{2}\right)\right) \quad \eta \text {-uniformly. }
$$




\section{Fourier Multipliers}

We write $\mathcal{F}=(u \mapsto \hat{u})$ for the Fourier transform on $\mathcal{S}^{\prime}\left(\mathbb{R}^{d}, E\right)$, the space of $E$-valued tempered distributions on $\mathbb{R}^{d}$, and $D:=-i \partial=-i\left(\partial_{1}, \ldots, \partial_{d}\right)$. If $a \in C\left(\mathbb{R}^{d}, \mathcal{L}(E)\right)$, then $a(D):=\mathcal{F}^{-1} a \mathcal{F}$ is the Fourier multiplier operator with symbol $a$. It is a linear map in $\mathcal{S}^{\prime}\left(\mathbb{R}^{d}, E\right)$ whose domain is the set of all $u \in \mathcal{S}^{\prime}\left(\mathbb{R}^{d}, E\right)$ with $a \hat{u} \in \mathcal{S}^{\prime}\left(\mathbb{R}^{d}, E\right)$. In particular,

$$
J_{\eta}^{z}:=\Lambda_{\eta}^{z}(D) \in \mathcal{L}\left(\mathcal{S}^{\prime}\left(\mathbb{R}^{d}, E\right)\right)
$$

The next two theorems form the fundament on which we build our proofs. Throughout this section, $\mathbb{X}=\mathbb{R}^{d}$.

Theorem 5.1. Let $\left(s_{0}, q\right)$ and $\left(s_{1}, q\right)$ be $\nu$-admissible. Then

$$
J_{\eta}^{s_{1}-s_{0}} \in \mathcal{L}\left(\mathfrak{F}_{q ; \eta}^{s_{1} / \nu}, \mathfrak{F}_{q ; \eta}^{s_{0} / \nu}\right) \quad \eta \text {-uniformly }
$$

Theorem 5.2. Let $(s, q)$ be $\nu$-admissible.

(i) Suppose $a_{\eta} \in \mathcal{M}_{\eta}(\mathcal{L}(E))$ for $\eta>0$. Then $a_{\eta}(D) \in \mathcal{L}\left(\mathfrak{F}_{q}^{s / \nu}\right)$ and

$$
\left\|a_{\eta}(D)\right\|_{\mathcal{L}\left(\mathfrak{F}_{q ; \eta}^{s / \nu}\right)} \leq c\left\|a_{\eta}\right\|_{\mathcal{M}_{\eta}} \quad \eta \text {-uniformly. }
$$

(ii) If $b_{\eta} \in \mathcal{M}_{\eta}(\mathcal{L}(E))$ for $\eta>0$, then $\left(a_{\eta} b_{\eta}\right)(D)=a_{\eta}(D) b_{\eta}(D)$.

Detailed proofs for these two theorems are given in [1] (see also [6] for some preliminary results not covering the case $q=\infty$ ). Here we restrict ourselves to some remarks.

(1) Consider the trivial weight system $[1,1,1]$. Let $\eta=1$ and assume $s$, $s_{0}$, and $s_{1}$ belong to $\mathbb{N}$ (so that $1<q<\infty$ by admissibility). Then $\mathfrak{F}_{q}^{s} \doteq H_{q}^{s}$, a Bessel potential space. In this case the 'lifting' Theorem 5.1 is well-known (e.g., [47], [48]). Its anisotropic version is contained in [6, Theorem 3.7.1].

In the isotropic, resp. anisotropic, case each $a \in \mathcal{M}(\mathcal{L}(E))$ is a Mikhlin, resp. Marcinkiewicz, multiplier. Thus, in the present setting, Theorem 5.2 follows by combining Theorem 5.1 with the Mikhlin, resp. Marcinkiewicz, multiplier theorem for $L_{q}\left(\mathbb{R}^{n}, E\right)$.

It should be noted that the $\nu$-admissibility assumption excludes the choices $q=1$ and $q=\infty$ for which these multiplier theorems do not hold.

(2) Let $s, s_{0}, s_{1} \notin \mathbb{N}$ and $1 \leq q \leq \infty$. If $q<\infty$, then $\mathfrak{F}_{q}^{s / \nu} \doteq B_{q, q}^{s / \nu}$, an anisotropic Besov space, and $\mathfrak{F}_{\infty}^{s / \nu} \doteq b_{\infty, \infty}^{s / \nu}$, an anisotropic little Besov space. Thus it follows that the above theorems are parameter-dependent anisotropic extensions of the corresponding results established in [4] in the isotropic case. As in that paper, $E$ can then be replaced by an arbitrary infinite-dimensional Banach space.

By combining these two theorems we arrive at multiplier theorems involving $\mathfrak{F}_{q}^{s / \nu}$-spaces of different order. 
Theorem 5.3. Let $\left(s_{0}, q\right)$ and $\left(s_{1}, q\right)$ be $\nu$-admissible.

(i) Assume $a_{\eta} \in C\left(\mathbb{R}^{d}, \mathcal{L}(E)\right)$ satisfies $\Lambda_{\eta}^{s_{0}-s_{1}} a_{\eta} \in \mathcal{M}_{\eta}(\mathcal{L}(E))$ for $\eta>0$. Then $a_{\eta}(D) \in \mathcal{L}\left(\mathfrak{F}_{q}^{s_{1} / \nu}, \mathfrak{F}_{q}^{s_{0} / \nu}\right)$ and

$$
\left\|a_{\eta}(D)\right\|_{\mathcal{L}\left(\mathfrak{F}_{q ; \eta}^{s_{1} / \nu}, \mathfrak{F}_{q ; \eta}^{s_{0} / \nu}\right)} \leq c\left\|\Lambda_{\eta}^{s_{0}-s_{1}} a_{\eta}\right\|_{\mathcal{M}_{\eta}} \quad \eta \text {-uniformly }
$$

(ii) If, in addition, $a_{\eta} \in C\left(\mathbb{R}^{d}, \mathcal{L}\right.$ aut $\left.(E)\right)$ with

$$
\Lambda_{\eta}^{s_{1}-s_{0}} a_{\eta}^{-1} \in B C\left(\mathbb{R}^{d}, \mathcal{L}(E)\right) \quad \eta \text {-uniformly }
$$

then $a_{\eta}(D) \in \mathcal{L} \operatorname{is}\left(\mathfrak{F}_{q}^{s_{1} / \nu}, \mathfrak{F}_{q}^{s_{0} / \nu}\right)$ with $a_{\eta}(D)^{-1}=a_{\eta}^{-1}(D)$ and

$$
\left\|a_{\eta}(D)^{-1}\right\|_{\mathcal{L}\left(\mathfrak{F}_{q ; \eta}^{s_{0} / \nu}, \mathfrak{F}_{q ; \eta}^{s_{1} / \nu}\right)} \leq c\left(\left\|\Lambda_{\eta}^{s_{0}-s_{1}} a_{\eta}\right\|_{\mathcal{M}_{\eta}},\left\|\Lambda_{\eta}^{s_{1}-s_{0}} a_{\eta}^{-1}\right\|_{\infty}\right)
$$

$\eta$-uniformly.

Proof. (1) We set $b_{\eta}:=\Lambda_{\eta}^{s_{0}-s_{1}} a_{\eta}$. Then the assumptions and Theorem 5.2 imply $b_{\eta}(D) \in \mathcal{L}\left(\mathfrak{F}_{q}^{s_{1} / \nu}\right)$ and

$$
\left\|b_{\eta}\right\|_{\mathcal{L}\left(\mathfrak{F}_{q ; \eta}^{s_{1} / \nu}\right)} \leq c\left\|\Lambda_{\eta}^{s_{0}-s_{1}} a_{\eta}\right\|_{\mathcal{M}_{\eta}} \quad \eta \text {-uniformly }
$$

Hence

$$
\begin{aligned}
a_{\eta}(D) u & =\mathcal{F}^{-1} a_{\eta} \mathcal{F} u=\mathcal{F}^{-1} \Lambda_{\eta}^{s_{1}-s_{0}} \Lambda_{\eta}^{s_{0}-s_{1}} a_{\eta} \mathcal{F} u \\
& =\mathcal{F}^{-1} \Lambda_{\eta}^{s_{1}-s_{0}} \mathcal{F}^{-1} \Lambda_{\eta}^{s_{0}-s_{1}} a_{\eta} \mathcal{F} u=J_{\eta}^{s_{1}-s_{0}} b_{\eta}(D) u
\end{aligned}
$$

for $u \in \mathfrak{F}_{q}^{s_{1}}$. Now (5.1) follows from (5.3) and Theorem 5.1,

(2) Let the additional hypothesis be satisfied. We obtain from (5.2) and Lemma 1.4.2 in [6] that $b_{\eta}^{-1} \in \mathcal{M}_{\eta}(\mathcal{L}(E))$ and

$$
\left\|b_{\eta}^{-1}\right\|_{\mathcal{M}_{\eta}} \leq c\left(\left\|b_{\eta}\right\|_{\mathcal{M}_{\eta}},\left\|b_{\eta}^{-1}\right\|_{\infty}\right) \quad \eta \text {-uniformly }
$$

Thus, as in step $(1), a_{\eta}^{-1}(D) \in \mathcal{L}\left(\mathfrak{F}_{q}^{s_{0} / \nu}, \mathfrak{F}_{q}^{s_{1} / \nu}\right)$ and

$$
\left\|a_{\eta}^{-1}(D)\right\|_{\mathcal{L}\left(\mathfrak{F}_{q ; \eta}^{s_{0} / \nu}, \mathfrak{F}_{q ; \eta}^{s_{1} / \nu}\right)} \leq c\left(\left\|\Lambda_{\eta}^{s_{0}-s_{1}} a_{\eta}\right\|_{\mathcal{M}_{\eta}},\left\|\Lambda_{\eta}^{s_{1}-s_{0}} a_{\eta}^{-1}\right\|_{\infty}\right)
$$

$\eta$-uniformly.

If $u \in \mathfrak{F}_{q}^{s_{1} / \nu}$, then

$$
a_{\eta}^{-1}(D) a_{\eta}(D) u=\mathcal{F}^{-1} a_{\eta}^{-1} \mathcal{F} \mathcal{F}^{-1} a_{\eta} \mathcal{F} u=\mathcal{F}^{-1} a_{\eta}^{-1} a_{\eta} \mathcal{F} u=u .
$$

Analogously,

$$
a_{\eta}(D) a_{\eta}^{-1}(D) v=\mathcal{F}^{-1} a_{\eta} \mathcal{F} \mathcal{F}^{-1} a_{\eta}^{-1} \mathcal{F} v=v, \quad v \in \mathcal{F}_{q}^{s_{0} / \nu},
$$

Now the assertion is clear.

Corollary 5.4. Let $\left(s_{0}, q\right)$ and $\left(s_{1}, q\right)$ be $\nu$-admissible.

(i) Suppose $a \in \mathcal{H}_{s_{1}-s_{0}}(\mathrm{Z}, \mathcal{L}(E))$. Then $a_{\eta}(D) \in \mathcal{L}\left(\mathfrak{F}_{q}^{s_{1} / \nu}, \mathfrak{F}_{q}^{s_{0} / \nu}\right)$ and

$$
\left\|a_{\eta}(D)\right\|_{\mathcal{L}\left(\mathfrak{F}_{q ; \eta}^{s_{1} / \nu}, \mathfrak{F}_{q ; \eta}^{s_{0} / \nu}\right)} \leq c\|a\|_{\mathcal{H}_{s_{1}-s_{0}}} \quad \eta \text {-uniformly. }
$$


(ii) Let $a \in \mathcal{H}_{s_{1}-s_{0}}(\mathrm{Z}, \mathcal{L}$ aut $(E))$ satisfy $a^{-1} \circ r_{\Lambda} \in B C([\Lambda=1], \mathcal{L}(E))$. Then $a_{\eta}(D) \in \mathcal{L} \operatorname{is}\left(\mathfrak{F}_{q}^{s_{1} / \nu}, \mathfrak{F}_{q}^{s_{0} / \nu}\right)$ with $a_{\eta}(D)^{-1}=a_{\eta}^{-1}(D)$ and

$$
\left\|a_{\eta}^{-1}(D)\right\|_{\mathcal{L}\left(\mathfrak{F}_{q ; \eta}^{s_{0} / \nu}, \mathfrak{F}_{q ; \eta}^{s_{1} / \nu}\right)} \leq c\left(\|a\|_{\mathcal{H}_{s_{1}-s_{0}}},\left\|a^{-1} \circ r_{\Lambda}\right\|_{\infty}\right) \quad \eta \text {-uniformly. }
$$

Proof. It follows from (4.4) and (4.5) that $\Lambda^{s_{0}-s_{1}} a \in \mathcal{H}_{0}(\mathrm{Z}, \mathcal{L}(E))$ and $\left\|\Lambda^{s_{0}-s_{1}} a\right\|_{\mathcal{H}_{0}} \leq c\|a\|_{\mathcal{H}_{s_{1}-s_{0}}}$. Hence the first assertion is a consequence of (4.7) and part (i) of the theorem. Now we get assertion (ii) by analogous arguments from (4.6).

\section{The Full-Space Model Case}

In this section we consider the flat case $(M, g)=\left(\mathbb{R}^{m}, g_{m}\right)$. We restrict ourselves to constant coefficient principal part operators. More precisely, we assume

$$
\left.\begin{array}{l}
\text { - } \mathcal{A}=\sum_{|a|=r} a_{\alpha} D^{\alpha}, a_{\alpha} \in \mathcal{L}(E) . \\
\text { - } \mathcal{A} \text { is normally } \varepsilon \text {-elliptic }
\end{array}\right\}
$$

for some $\varepsilon \in(0,1]$. We set

$$
\mathrm{a}:=\sum_{|a|=r}\left|a_{\alpha}\right|_{\mathcal{L}(E)}
$$

and note that $|\mathfrak{s} \mathcal{A}(\xi)|_{\mathcal{L}(E)} \leq$ a for $|\xi|=1$. We fix a constant $\bar{\kappa}$ satisfying $\mathrm{a}+\varepsilon^{-1} \leq \bar{\kappa}$. We set $d:=m$, endow $\mathbb{R}^{m}$ with the trivial weight system, and equip $\mathrm{Z}:=\mathbb{R}^{m} \times \mathbb{R}^{+}$with the 1 -augmentation of it. Then we put

$$
a(\zeta):=\eta^{r}+\mathfrak{s} \mathcal{A}(\xi), \quad \zeta=(\xi, \eta) \in \mathrm{Z} .
$$

Observe that $\Lambda(\zeta)=\left(|\xi|^{2}+\eta^{2}\right)^{1 / 2}$ and

$$
a \in \mathcal{H}_{r}(\mathrm{Z}, \mathcal{L}(E)), \quad\|a\|_{\mathcal{H}_{r}} \leq c(\mathrm{a}) .
$$

As usual, $\rho(A):=\mathbb{C} \backslash \sigma(A)$ is the resolvent set of a linear operator $A$.

Lemma 6.1. $[\operatorname{Re} z \geq 0] \subset \rho(-a(\zeta))$ and

$$
\left|(\lambda+a(\zeta))^{-1}\right|_{\mathcal{L}(E)} \leq c(\bar{\kappa})\left(\Lambda^{r}(\zeta)+|\lambda|\right)^{-1}
$$

for $\operatorname{Re} \lambda \geq 0$ and $\zeta \in \dot{Z}$.

Proof. By the normal $\varepsilon$-ellipticity and the $r$-homogeneity of $\mathfrak{s} \mathcal{A}$ we get

$$
\sigma(a(\zeta)) \subset\left[\operatorname{Re} z \geq \varepsilon \Lambda^{r}(\zeta)\right], \quad \zeta \in \dot{\mathrm{Z}} .
$$

Let $\Lambda(\zeta)=1$. If $|\xi|^{2} \geq 1 / 2$, then

$$
\sigma(a(\zeta)) \subset\left[\operatorname{Re} z \geq \varepsilon / 2^{r / 2}\right] .
$$

Otherwise, $\eta^{2} \geq 1 / 2$ and (6.3) applies as well. 
Suppose $z \in \sigma(\lambda+a(\zeta))$ with $\operatorname{Re} \lambda \geq 0$ and $\Lambda(\zeta)=1$. Then $z=\lambda+\mu$ with $\mu \in \sigma(a(\zeta))$. Hence $|\mu| \geq \operatorname{Re} \mu \geq \varepsilon / 2^{r / 2}$ by (6.3). Since $\operatorname{det}(\lambda+a(\zeta))$ equals the product of the eigenvalues of $\lambda+a(\zeta)$, counted with multiplicities,

$$
|\operatorname{det}(\lambda+a(\zeta))| \geq\left(\varepsilon / 2^{r / 2}\right)^{N}, \quad \operatorname{Re} \lambda \geq 0, \quad \zeta \in[\Lambda=1],
$$

where $N=\operatorname{dim}(E)$. Now we deduce from Cramer's rule (e.g., [32, (I.4.12]) that $\lambda \in \rho(-a(\zeta))$ and

$$
\left|(\lambda+a(\zeta))^{-1}\right|_{\mathcal{L}(E)} \leq c(\bar{\kappa}), \quad \zeta \in[\Lambda=1],
$$

provided $\operatorname{Re} \lambda \geq 0$ with $|\lambda| \leq 2(1+a)$. If $|\lambda| \geq 2(1+a) \geq 2\left\|a \circ r_{\Lambda}\right\|_{\infty}$, then a Neumann series argument shows that

$$
|\lambda|\left|(\lambda+a(\zeta))^{-1}\right|_{\mathcal{L}(E)}=\left|\left(1+\lambda^{-1} a(\zeta)\right)^{-1}\right|_{\mathcal{L}(E)} \leq 2, \quad \zeta \in[\Lambda=1] .
$$

By combining this with (6.4) we find

$$
\left|(\lambda+a(\zeta))^{-1}\right|_{\mathcal{L}(E)} \leq c(\bar{\kappa})(1+|\lambda|)^{-1}, \quad \operatorname{Re} \lambda \geq 0, \quad \Lambda(\zeta)=1 .
$$

Now the assertion follows from $\lambda+a=\Lambda^{r}\left(\Lambda^{-r} \lambda+a \circ r_{\Lambda}\right)$.

We set $\tilde{d}:=d+1=m+1$ and consider the $r$-parabolic weight system $[\tilde{\ell}, \tilde{\boldsymbol{d}}, \tilde{\boldsymbol{\nu}}]=[2,(m, 1),(1, r)]$ on $\mathbb{R}^{\tilde{d}}=\mathbb{R}^{m} \times \mathbb{R}$. Then we set

$$
\mathfrak{F}_{q ; \eta}^{s / \vec{r}}:=\mathfrak{F}_{q ; \eta}^{s / \tilde{\nu}}\left(\mathbb{R}^{m} \times \mathbb{R}, E\right) .
$$

We also let $\mathcal{A}_{\eta}:=\eta+\mathcal{A}$ and study the normally $\varepsilon$-parabolic differential operator $\partial_{t}+\mathcal{A}_{\eta}$ on $\mathbb{R}^{m} \times \mathbb{R}$.

Theorem 6.2. Let $(s, q)$ be r-admissible. Then $\partial_{t}+\mathcal{A}_{\eta}$ is an element of $\mathcal{L} \operatorname{is}\left(\mathfrak{F}_{q}^{(s+r) / \vec{r}}, \mathfrak{F}_{q}^{s / \vec{r}}\right)$ and

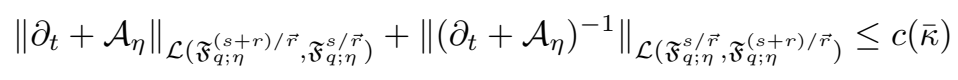

$\eta$-uniformly.

Proof. We endow $\tilde{Z}:=\mathbb{R}^{m} \times \mathbb{R} \times \mathbb{R}^{+}$with the $r$-augmentation of $[\tilde{\ell}, \tilde{\boldsymbol{d}}, \tilde{\boldsymbol{\nu}}]$. Then $r=\operatorname{LCM}(\tilde{\boldsymbol{\nu}})$, and the natural quasinorm on $\tilde{Z}$ is given by

$\tilde{\Lambda}(\tilde{\zeta})=\left(|\xi|^{2 r}+|\tau|^{2}+\eta^{2}\right)^{1 / 2 r} \sim\left(\Lambda^{2 r}\left(\xi, \eta^{1 / r}\right)+|\tau|^{2}\right)^{1 / 2 r}, \quad \tilde{\zeta}=(\xi, \tau, \eta) \in \tilde{Z}$, with $\zeta=(\xi, \eta) \in$ Z. We set

$$
\tilde{a}(\tilde{\zeta}):=-i \tau+\eta+\mathfrak{s} \mathcal{A}(\xi) .
$$

It is obvious that

$$
\tilde{a} \in \tilde{\mathcal{H}}_{r}:=\mathcal{H}_{r}(\tilde{\mathrm{Z}}, \mathcal{L}(E)), \quad\|\tilde{a}\|_{\tilde{\mathcal{H}}_{r}} \leq c(\bar{\kappa}) .
$$

Since $\tilde{a}(\tilde{\zeta})=-i \tau+a\left(\xi, \eta^{1 / r}\right)$, it follows from Lemma 6.1 that it is invertible for $\tilde{\zeta} \neq 0$ and

$$
\left|\tilde{a}^{-1}(\tilde{\zeta})\right|_{\mathcal{L}(E)} \leq c(\bar{\kappa})\left(\Lambda^{r}\left(\xi, \eta^{1 / r}\right)+|\tau|\right)^{-1} \leq c(\bar{\kappa}) \tilde{\Lambda}^{-r}(\tilde{\zeta}) .
$$


Thus $\left\|\tilde{a}^{-1} \circ r_{\tilde{\Lambda}}\right\|_{\infty} \leq c(\bar{\kappa})$. Hence we infer from (4.6) and (6.5) that $\tilde{a}^{-1} \in \tilde{\mathcal{H}}_{-r}$ and $\left\|\tilde{a}^{-1}\right\|_{\tilde{\mathcal{H}}_{-r}} \leq c(\bar{\kappa})$. Now the assertion is a consequence of Corollary 5.4 and the fact that $\tilde{a}_{\eta}(\tilde{D})=\partial_{t}+\mathcal{A}_{\eta}$, where $\tilde{D}:=\left(D, D_{t}\right)$.

\section{The Semigroup}

We continue to presuppose conditions (6.1) and use the notations of the preceding section. Then $\mathfrak{F}_{q}^{s}=\mathfrak{F}_{q}^{s}\left(\mathbb{R}^{m}, E\right)$.

Theorem 7.1. Let $(s, q)$ be 1-admissible. Then $\mathcal{A}_{\eta} \in \mathcal{L} \operatorname{is}\left(\mathfrak{F}_{q}^{s+r}, \mathfrak{F}_{q}^{s}\right)$, the half-plane $[\operatorname{Re} z \geq 0]$ is contained in $\rho\left(-\mathcal{A}_{\eta}\right)$, and

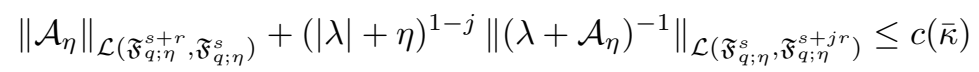

for $\operatorname{Re} \lambda \geq 0, \eta>0$, and $j=0,1$.

Proof. First we infer from (6.2), (4.4), and (4.5) that $\Lambda^{-r} a \in \mathcal{H}_{0}(\mathrm{Z}, \mathcal{L}(E))$ and $\left\|\Lambda^{-r} a\right\|_{\mathcal{H}_{0}} \leq c(\mathrm{a})$. Hence, by Corollary 5.4(i),

$$
a_{\eta}(D) \in \mathcal{L}\left(\mathfrak{F}_{q}^{s+r}, \mathfrak{F}_{q}^{s}\right), \quad\left\|a_{\eta}(D)\right\|_{\mathcal{L}\left(\mathfrak{F}_{q ; \eta}^{s+r}, \mathfrak{F}_{q ; \eta}^{s}\right)} \leq c(\mathrm{a})
$$

$\eta$-uniformly. Using (6.2) once more, we obtain from (4.3) and Lemma 6.1 that

$$
\Lambda^{\beta \cdot \boldsymbol{\omega}}\left|\left(\partial_{\xi}^{\beta} a\right)(\lambda+a)^{-1}\right|_{\mathcal{L}(E)}(\zeta) \leq c(\mathrm{a}) \Lambda^{r}(\zeta)\left(\Lambda^{r}(\zeta)+|\lambda|\right)^{-1} \leq c(\mathrm{a})
$$

for $\beta \in \mathbb{N}^{m}, \zeta \in \dot{Z}$, and Re $\lambda \geq 0$. From this, 6. Lemma 1.4.2], and Lemma6.1 we get

$$
\begin{aligned}
\Lambda^{\alpha \cdot \boldsymbol{\omega}}(\zeta)\left|\partial_{\xi}^{\alpha}(\lambda+a)^{-1}(\zeta)\right|_{\mathcal{L}(E)} & \leq c(\mathrm{a})\left|(\lambda+a(\zeta))^{-1}\right|_{\mathcal{L}(E)} \\
& \leq c(\bar{\kappa})\left(\Lambda^{r}(\zeta)+|\lambda|\right)^{-1}
\end{aligned}
$$

for $\alpha \in \mathbb{N}^{m}, \quad \zeta \in \dot{Z}$, and $\operatorname{Re} \lambda \geq 0$. Using (4.4), (4.2), (7.3), and Leibniz' rule, we find

$$
\Lambda^{\alpha \cdot \boldsymbol{\omega}}\left|\partial_{\xi}^{\alpha}\left(\Lambda^{r}(\lambda+a)^{-1}\right)\right|_{\mathcal{L}(E)}(\zeta) \leq c(\bar{\kappa}) \Lambda^{r}(\zeta)\left(\Lambda^{r}(\zeta)+|\lambda|\right)^{-1} \leq c(\bar{\kappa})
$$

for $\alpha \in \mathbb{N}^{m}$ with $\alpha \cdot \boldsymbol{\omega} \leq 2|\boldsymbol{\omega}|=2 m, \quad \zeta \in \dot{\mathrm{Z}}$, and $\operatorname{Re} \lambda \geq 0$.

Note that (7.3) guarantees

$$
\left(\lambda+a_{\eta}\right)^{-1} \in \mathcal{M}_{\eta}\left(\mathbb{R}^{m}, \mathcal{L}(E)\right), \quad\left\|\left(\lambda+a_{\eta}\right)^{-1}\right\|_{\mathcal{M}_{\eta}} \leq c(\bar{\kappa})\left(|\lambda|+\eta^{r}\right)^{-1}
$$

$\eta$-uniformly for $\operatorname{Re} \lambda \geq 0$. Similarly, by (7.4),

$$
\Lambda_{\eta}^{r}\left(\lambda+a_{\eta}\right)^{-1} \in \mathcal{M}_{\eta}\left(\mathbb{R}^{m}, \mathcal{L}(E)\right), \quad\left\|\Lambda_{\eta}^{r}\left(\lambda+a_{\eta}\right)^{-1}\right\|_{\mathcal{M}_{\eta}} \leq c(\bar{\kappa})
$$

$\eta$-uniformly for $\operatorname{Re} \lambda \geq 0$. Hence, by Theorem 5.2 ,

$$
\left(\lambda+a_{\eta}\right)^{-1}(D) \in \mathcal{L}\left(\mathfrak{F}_{\eta}^{s}\right), \quad\left\|\left(\lambda+a_{\eta}\right)^{-1}(D)\right\|_{\mathcal{L}\left(\mathfrak{F}_{q ; \eta}^{s}\right)} \leq c(\bar{\kappa})\left(|\lambda|+\eta^{r}\right)^{-1}
$$

$\eta$-uniformly, and, similarly,

$$
J_{\eta}^{r}\left(\lambda+a_{\eta}\right)^{-1}(D)=\left(\Lambda_{\eta}^{r}\left(\lambda+a_{\eta}\right)^{-1}\right)(D) \in \mathcal{L}\left(\mathfrak{F}_{q}^{s}\right)
$$


and, due to Theorem 5.1 .

$$
\left\|\left(\lambda+a_{\eta}\right)^{-1}(D)\right\|_{\mathcal{L}\left(\mathfrak{F}_{q ; \eta}^{s}, \mathfrak{F}_{q ; \eta}^{s+r}\right)} \leq c\left\|J_{\eta}^{r}\left(\lambda+a_{\eta}\right)^{-1}(D)\right\|_{\mathcal{L}\left(\mathfrak{F}_{q ; \eta}^{s}\right)} \leq c(\bar{\kappa})
$$

$\eta$-uniformly for $\operatorname{Re} \lambda \geq 0$. Using (7.2) we find, similarly as in the proof of Theorem [5.1, that $\left(\lambda+a_{\eta}\right)^{-1}(D)=\left(\lambda+a_{\eta}(D)\right)^{-1}$. Now the assertion follows from (7.2), $\mathcal{A}_{\eta}=a_{\eta^{1 / r}}(D)$, and (7.5).

Corollary 7.2. Let $(s, q)$ be 1-admissible. Then $\mathcal{A}_{\eta} \in \mathcal{H}\left(\mathfrak{F}_{q}^{s+r}, \mathfrak{F}_{q}^{s}\right)$ and the semigroup $\left\{e^{-t \mathcal{A}_{\eta}} ; t \geq 0\right\}$ is exponentially decaying.

Proof. Since $\mathcal{A}_{\eta} \in \mathcal{L}$ is $\left(\mathfrak{F}_{q}^{s+r}, \mathfrak{F}_{q}^{s}\right)$, it follows that $\mathcal{A}_{\eta}$ is closed if we consider it as a linear operator in $\mathfrak{F}_{q}^{s}$ with domain $\mathfrak{F}_{q}^{s+r}$ (cf. [3, Lemma I.1.1.2]). Moreover, it is then densely defined, due to (1.12) and (1.14). Now $\mathcal{A}_{\eta} \in \mathcal{H}\left(\mathfrak{F}_{q}^{s+r}, \mathfrak{F}_{q}^{s}\right)$ is a well-known consequence of the resolvent estimate contained in (7.1).

From semigroup theory it is known that there exists $\varphi \in(\pi / 2, \pi)$ such that $[|\arg z| \leq \varphi] \subset \rho\left(-\mathcal{A}_{\eta}\right)$. From this and the fact that $0 \in \rho\left(-\mathcal{A}_{\eta}\right)$ it follows that there exists $\gamma=\gamma(\eta)>0$ such that $\sigma\left(-\mathcal{A}_{\eta}\right) \subset[\operatorname{Re} z \leq-\gamma]$, that is, the spectral bound of $-\mathcal{A}_{\eta}$ is negative. Hence the growth bound is negative too.

Proposition 7.3. Let $(s, q)$ be $r$-admissible. If $f \in \mathfrak{F}_{q}^{s / \vec{r}}$, then

$$
\left(\partial_{t}+\mathcal{A}_{\eta}\right)^{-1} f=\int_{-\infty}^{t} e^{-(t-\tau) \mathcal{A}_{\eta}} f(\tau) d \tau, \quad \text { a.a. } t \in \mathbb{R} .
$$

Proof. (1) We fix $\eta>0$ and set $U(t)=V(t):=e^{-t \mathcal{A}_{\eta}}$ for $t \geq 0$, and $V(t)=0$ for $t<0$. Since the semigroup $\{U(t) ; t \geq 0\}$ is exponentially decaying, it follows that $V$ belongs to $L_{1}\left(\mathbb{R}, \mathcal{L}\left(\mathfrak{F}_{q}^{s}\right)\right)$. Hence, by Young's inequality,

$$
(g \mapsto V * g) \in \mathcal{L}\left(L_{q}(\mathbb{R}), \mathfrak{F}_{q}^{s}\right), \quad 1 \leq q \leq \infty,
$$

and

$$
V * g(t)=\int_{-\infty}^{t} U(t-\tau) g(\tau) d \tau, \quad \text { a.a. } t \in \mathbb{R},, \quad g \in L_{1}\left(\mathbb{R}, \mathfrak{F}_{q}^{s}\right) .
$$

This remains valid if $L_{\infty}$ is replaced by $B U C$.

It is a consequence of Theorem 7.1 that $\|\cdot\|_{\mathfrak{F}_{q}^{s+r}} \sim\left\|\mathcal{A}_{\eta} \cdot\right\|_{\mathfrak{F}_{q}^{s}}$. Thus we infer from Corollary 7.2 that $\{U(t) ; t \geq 0\}$ restricts to a strongly continuous exponentially decaying analytic semigroup on $\mathfrak{F}_{q}^{s+r}$ (e.g., [3, Theorem V.2.1.3]).

(2) Assume $g \in B U C\left(\mathbb{R}, \mathfrak{F}_{q}^{s+r}\right)$. Then the arguments of step (1) show that $v:=V * g$ belongs to $B U C\left(\mathbb{R}, \mathfrak{F}_{q}^{s+r}\right)$. Given $h>0$,

$$
v(t+h)-v(t)=\int_{t}^{t+h} U(t+h-\tau) g(\tau) d \tau+(U(h)-1) v(t), \quad t \in \mathbb{R}
$$

From this we deduce that the right derivative $\partial_{t}^{+} v$ exists in $\mathfrak{F}_{q}^{s}$ and equals $g-\mathcal{A}_{\eta} v$. Since this function is continuous, $v \in C^{1}\left(\mathbb{R}, \mathfrak{F}_{q}^{s}\right)$ and $\left(\partial_{t}+\mathcal{A}_{\eta}\right) v=g$, that is, $\left(\partial_{t}+\mathcal{A}_{\eta}\right)^{-1} g=V * g$. 
(3) Suppose $q<\infty$. Then, see [1] or [6],

$$
\mathcal{S}\left(\mathbb{R}, \mathcal{S}\left(\mathbb{R}^{m}, E\right)\right) \stackrel{d}{\hookrightarrow} \mathcal{S}\left(\mathbb{R}^{m} \times \mathbb{R}, E\right) \stackrel{d}{\hookrightarrow} W_{q}^{(s+r) / \vec{r}} \stackrel{d}{\hookrightarrow} W_{q}^{s / \vec{r}}
$$

and

$$
\mathcal{S}\left(\mathbb{R}, \mathcal{S}\left(\mathbb{R}^{m}, E\right)\right) \hookrightarrow \mathcal{S}\left(\mathbb{R}, W_{q}^{s+r}\right) \hookrightarrow B U C\left(\mathbb{R}, W_{q}^{s+r}\right) .
$$

Thus, if $f \in W_{q}^{s / \vec{r}}$, there exists a sequence $\left(f_{j}\right)$ in $W_{q}^{s / \vec{r}} \cap B U C\left(\mathbb{R}, W_{q}^{s+r}\right)$ converging in $W_{q}^{s / \vec{r}}$, hence, by Theorem 3.2 in $L_{q}\left(\mathbb{R}, W_{q}^{s}\right)$, towards $f$. By step (2), $\left(\partial_{t}+\mathcal{A}_{\eta}\right)^{-1} f_{j}=V * f_{j}$ for $j \in \mathbb{N}$. It follows from (7.7) that $V * f_{j} \rightarrow V * f$ in $L_{q}\left(\mathbb{R}, W_{q}^{s}\right)$. Theorem 6.2 implies that $\left(\partial_{t}+\mathcal{A}_{\eta}\right)^{-1} f_{j} \rightarrow\left(\partial_{t}+\mathcal{A}_{\eta}\right)^{-1} f$ in $W_{q}^{(s+r) / \vec{r}}$, hence in $L_{q}\left(\mathbb{R}, W_{q}^{s}\right)$. Consequently, $\left(\partial_{t}+\mathcal{A}_{\eta}\right)^{-1} f=V * f$, which proves the assertion in this case.

(4) Assume $q=\infty$ and $f \in b u c^{s / \vec{r}}$. We see from $b u c^{(s+r) / \vec{r}} \stackrel{d}{\hookrightarrow} b u c^{s / \vec{r}}$ that there exists a sequence $\left(f_{j}\right)$ in buc $(s+r) / \vec{r}$ converging in $b u c^{s / \vec{r}}$, hence, once more by Theorem 3.2 in $B U C\left(\mathbb{R}, b u c^{s}\right)$, towards $f$. Since $f_{j}$ belongs to $B U C\left(\mathbb{R}, b u c^{s+r}\right)$ by Theorem 3.2 , we get from step $(2)$ that $\left(\partial_{t}+\mathcal{A}_{\eta}\right)^{-1} f_{j}$ equals $V * f_{j}$ for $j \in \mathbb{N}$. This implies $\left(\partial_{t}+\mathcal{A}_{\eta}\right)^{-1} f=V * f$ by the arguments of the preceding step.

Corollary 7.4. Let $(s, q)$ be $r$-admissible. Let either (3.2) be satisfied or suppose $0 \leq s<r / q$ and set $\mathfrak{F}_{q}^{s / \vec{r}}(\stackrel{H}{H}, E):=\mathfrak{F}_{q}^{s / \vec{r}}(\mathbb{H}, E)$. Then

$$
\mathfrak{R} \circ\left(\partial_{t}+\mathcal{A}_{\eta}\right)^{-1} \circ \stackrel{\circ}{\mathfrak{E}} \in \mathcal{L}\left(\mathfrak{F}_{q ; \eta}^{s / \vec{r}}(\stackrel{\circ}{\mathbb{H}}, E), \mathfrak{F}_{q ; \eta}^{(s+r) / \vec{r}}(\stackrel{\circ}{\mathbb{H}}, E)\right)
$$

7-uniformly.

Proof. It follows from Corollary 3.7 and Theorem 6.2 that

$$
\left(\partial_{t}+\mathcal{A}_{\eta}\right)^{-1} \circ \stackrel{\circ}{\mathfrak{E}} \in \mathcal{L}\left(\mathfrak{F}_{q ; \eta}^{s / \vec{r}}(\stackrel{\circ}{\mathbb{H}}, E), \mathfrak{F}_{q ; \eta}^{(s+r) / \vec{r}}\right) \quad \eta \text {-uniformly. }
$$

Given $\stackrel{\mathfrak{E} f}{f} \in{ }_{0} \mathfrak{F}_{q}^{s / \vec{r}}$, we read off (17.6) that

$$
u(t):=\left(\partial_{t}+\mathcal{A}_{\eta}\right)^{-1} \circ \stackrel{\circ}{\mathfrak{E}} f(t)=0, \quad \text { a.a. } t<0 .
$$

Note that $(s+r) / r>1+k+1 / q$, where $k:=-1$ if $s<r / q$. Hence Theorem 3.2 and the (Banach-space-valued) Sobolev embedding theorem imply

$$
\mathfrak{F}_{q}^{(s+r) / \vec{r}} \hookrightarrow \mathfrak{F}_{q}^{(s+r) / r}\left(\mathbb{R}, L_{q}\left(\mathbb{R}^{m}, E\right)\right) \hookrightarrow C^{k+1}\left(\mathbb{R}, L_{q}\left(\mathbb{R}^{m}, E\right)\right) .
$$

From this and (7.8) we infer that $\vec{\gamma}^{k+1} u=0$. Now the claim follows.

\section{Cauchy Problems}

Now we turn to the Cauchy problem

$$
\left(\partial_{t}+\mathcal{A}_{\eta}\right) u=f \text { on } \mathbb{H}, \quad \gamma u=u_{0} \text { on } \partial \mathbb{H},
$$

retaining assumption (6.1). 
Theorem 8.1. Let $(s, q)$ be r-admissible. Then

$$
\left(\partial_{t}+\mathcal{A}_{\eta}, \gamma\right) \in \mathcal{L} \operatorname{is}\left(\mathfrak{F}_{q ; \eta}^{(s+r) / \vec{r}}(\mathbb{H}, E), \mathfrak{F}_{q ; \eta}^{s / \vec{r}}(\mathbb{H}, E) \times \mathfrak{F}_{q ; \eta}^{s+r(1-1 / q)}\right)
$$

$\eta$-uniformly with $c(\bar{\kappa})$-bounds, that is, $\left(\partial_{t}+\mathcal{A}_{\eta}, \gamma_{0}\right)$ and $\left(\partial_{t}+\mathcal{A}_{\eta}, \gamma_{0}\right)^{-1}$ are bounded by $c(\bar{\kappa})$, uniformly with respect to $\eta>0$.

Proof. (1) We write $M_{\eta}$, resp. $L_{\eta}$, for $\partial_{t}+\mathcal{A}_{\eta}$ if this operator is considered on $\mathbb{R}^{m} \times \mathbb{R}$, resp. $\mathbb{H}$. Let $(\mathfrak{R}, \mathfrak{E})$ be the r-e pair of Theorem 3.6 for $d=m+1$. Then Theorems 3.6, 6.2, and 3.5 imply

$$
\left(\Re \circ M_{\eta} \circ \mathfrak{E}, \gamma\right) \in \mathcal{L}\left(\mathfrak{F}_{q ; \eta}^{(s+r) / \vec{r}}(\mathbb{H}, E), \mathfrak{F}_{q ; \eta}^{s / \vec{r}}(\mathbb{H}, E) \times \mathfrak{F}_{q ; \eta}^{s+r(1-1 / q)}\right)
$$

$\eta$-uniformly with $c(\bar{\kappa})$-bounds. Since $\mathfrak{R}$ commutes with $\partial^{\alpha}$ and $\partial_{t}$, we see

$$
L_{\eta}=\mathfrak{R} \circ M_{\eta} \circ \mathfrak{E} .
$$

(2) Let $k \in \mathbb{N}$ and suppose

$$
r(k+1 / q)<s<r(k+1+1 / q), \quad s \notin(\mathbb{N}+1 / q) \cup(\mathbb{N}+r / q) .
$$

Then $s+r(1-j-1 / q)$ is $r$-admissible and, by Theorem 3.1(ii),

$$
\mathcal{A}_{\eta} \in \mathcal{L}\left(\mathfrak{F}_{q ; \eta}^{s+r(1-j-1 / q)}, \mathfrak{F}_{q ; \eta}^{s-r(j+1 / q)}\right)
$$

$\eta$-uniformly with $c($ a)-bounds for $0 \leq j \leq k$.

Suppose $u \in \mathfrak{F}_{q}^{(s+r) / \vec{r}}(\mathbb{H}, E)$ and set $f:=L_{\eta} u$. Then we get from Theorem 3.5 and (8.2)

$$
\gamma^{j+1} u=\partial_{t}^{j+1} u(0)=\partial_{t}^{j} f(0)-\mathcal{A}_{\eta} \partial_{t}^{j} u(0) \in \mathfrak{F}_{q}^{s-r(j+1 / q)}
$$

and

$$
\left\|\gamma^{j+1} u\right\|_{\mathfrak{F}_{q ; \eta}^{s-r(j+1 / q)}} \leq c(\mathrm{a})\|(f, \gamma u)\|_{\mathfrak{F}_{q ; \eta}^{s / \vec{r}}(\mathbb{H}, E) \times \mathfrak{F}_{q ; \eta}^{s+r(1-1 / q)}}
$$

for $0 \leq j \leq k$ and $\eta>0$.

Assume $\left(L_{\eta} u, \gamma u\right)=(0,0)$. Then we see from (8.1) and (8.4) that $u$ belongs to $\mathfrak{F}_{q}^{(s+r) / \vec{r}}(\mathbb{H}, E)$. Hence its trivial extension $\tilde{u}:=\mathfrak{E} u$ lies in $\mathfrak{F}_{q}^{(s+r) / \vec{r}}$ and satisfies $M_{\eta} \tilde{u}=0$. Consequently, $\tilde{u}=0$ by Theorem 6.2 Thus, taking Theorem 3.6 (ii) into consideration, $u=\mathfrak{R} \tilde{u}=0$. This shows that $\left(L_{\eta}, \gamma\right)$ is injective.

(3) Keeping assumption (8.1), we let $\left(f, u_{0}\right) \in \mathfrak{F}_{q}^{s / \vec{r}}(\mathbb{H}, E) \times \mathfrak{F}_{q}^{s+r(1-1 / q)}$. Define $u_{j ; \eta}$ for $0 \leq j \leq k$ inductively by

$$
u_{0 ; \eta}:=u_{0}, \quad u_{j+1 ; \eta}:=\gamma^{j} f-\mathcal{A}_{\eta} u_{j ; \eta} .
$$

It follows from (8.2) and Theorem 3.5 (i) that

$$
u_{j ; \eta} \in \mathfrak{F}_{q ; \eta}^{s-r(j+1 / q)}, \quad 0 \leq j \leq k,
$$

$\eta$-uniformly with $c($ a)-bounds.

We set

$$
\overrightarrow{\mathfrak{F}}_{q}^{s+r(1-1 / q)}:=\prod_{j=0}^{k} \mathfrak{F}_{q}^{s-r(j+1 / q)}, \quad V_{\eta}\left(f, u_{0}\right):=\left(u_{0 ; \eta}, \ldots, u_{k ; \eta}\right) .
$$


Then

$$
V_{\eta} \in \mathcal{L}\left(\mathfrak{F}_{q ; \eta}^{s / \vec{r}}(\mathbb{H}, E) \times \mathfrak{F}_{q ; \eta}^{s+r(1-1 / q)}, \overrightarrow{\mathfrak{F}}_{q}^{s+r(1-1 / q)}\right)
$$

$\eta$-uniformly with $c($ a)-bounds. Theorem 3.5(i) guarantees the existence of an $\eta$-uniform coretraction $\left(\vec{\gamma}^{k}\right)^{c}$ for the trace operator

$$
\vec{\gamma}^{k} \in \mathcal{L}\left(\mathfrak{F}_{q ; \eta}^{(s+r) / \vec{r}}(\mathbb{H}), \overrightarrow{\mathfrak{F}}_{q ; \eta}^{s+r(1-1 / q)}\right) .
$$

Hence

$$
W_{\eta}:=(\vec{\gamma})^{c} \circ V_{\eta} \in \mathcal{L}\left(\mathfrak{F}_{q ; \eta}^{s / \vec{r}}(\mathbb{H}, E) \times \mathfrak{F}_{q ; \eta}^{s+r(1-1 / q)}, \mathfrak{F}_{q ; \eta}^{(s+r) / \vec{r}}(\mathbb{H}, E)\right)
$$

$\eta$-uniformly with $c($ a)-bounds.

Let

$$
w_{\eta}:=W_{\eta}\left(f, u_{0}\right), \quad g_{\eta}:=f-L_{\eta} w_{\eta} .
$$

Then $w_{\eta} \in \mathfrak{F}_{q}^{(s+r) / \vec{r}}(\mathbb{H}, E)$ and (8.3) imply

$$
\gamma^{j} g_{\eta}=\gamma^{j} f-\gamma^{j} L_{\eta} w_{\eta}=\gamma^{j} f-\gamma^{j+1} w_{\eta}-\mathcal{A}_{\eta} \gamma^{j} w_{\eta}=0, \quad 0 \leq j \leq k .
$$

Hence $v_{\eta}:=\mathfrak{R} \circ M_{\eta}^{-1} \circ \stackrel{\circ}{\mathfrak{E}} g_{\eta} \in \mathfrak{F}^{(s+r) / \vec{r}}(\stackrel{\mathbb{H}}{1}, E)$ by Corollary 7.4 The second part of Theorem 3.6(i) implies $L_{\eta} \circ \mathfrak{R}=\mathfrak{R} \circ M_{\eta}$. Consequently,

$$
L_{\eta} v_{\eta}=\mathfrak{R} \circ \stackrel{\circ}{\mathfrak{E}} g_{\eta}=f-L_{\eta} w_{\eta}, \quad \gamma v_{\eta}=0 .
$$

Hence $u_{\eta}:=v_{\eta}+w_{\eta}$ satisfies $L_{\eta} u_{\eta}=f$ on $M \times \mathbb{R}^{+}$and $\gamma u_{\eta}=u_{0}$. This shows that $\left(L_{\eta}, \gamma\right)$ is surjective, thus bijective, and

$$
\left(L_{\eta}, \gamma\right)^{-1}\left(f, u_{0}\right)=\mathfrak{R} \circ M_{\eta}^{-1} \circ \stackrel{\circ}{\mathfrak{E}}\left(f-L_{\eta} W_{\eta}\left(f, u_{0}\right)\right)+W_{\eta}\left(f, u_{0}\right) .
$$

This implies the assertion in this case.

(4) Assume $0 \leq s<r / q$. In this case analogous arguments result in

$$
\left(L_{\eta}, \gamma\right)\left(f, u_{0}\right)=\mathfrak{R} \circ M_{\eta}^{-1} \circ \stackrel{\circ}{\mathfrak{E}}\left(f-L_{\eta} \gamma^{c} u_{0}\right)+\gamma^{c} u_{0}
$$

Thus the claim holds in this case too.

(5) Suppose $s \in(\mathbb{N}+1 / q) \cup(\mathbb{N}+r / q)$. We fix $s_{0}<s<s_{1}$ such that $\left(s_{0}, q\right)$ and $\left(s_{1}, q\right)$ are $r$-admissible and $s_{0}, s_{1} \notin(\mathbb{N}+1 / q) \cup(\mathbb{N}+r / q)$. Then, setting $\theta:=\left(s-s_{0}\right) /\left(s_{1}-s_{0}\right)$, the assertion follows by interpolation, due to Theorem 3.4 from what has just been shown. The theorem is proved.

\section{Localizations of Function Spaces}

We assume that the topological space underlying $M$ is separable and metrizable. Let $Q:=(-1,1) \subset \mathbb{R}$. If $\kappa$ is a local chart for $M$, then we write $U_{\kappa}$ for the corresponding coordinate patch $\operatorname{dom}(\kappa)$. A local chart $\kappa$ is normalized if $\kappa\left(U_{\kappa}\right)=Q^{m}$ whenever $U_{\kappa} \subset \stackrel{\circ}{M}$, the interior of $M$, and $\kappa\left(U_{\kappa}\right)=Q^{m} \cap \mathbb{H}^{m}$ if $U_{\kappa} \cap \partial M \neq \emptyset$.

An atlas $\mathfrak{K}$ for $M$ has finite multiplicity if there exists $k \in \mathbb{N}$ such that any intersection of more than $k$ coordinate patches is empty. In this case

$$
\mathfrak{N}(\kappa):=\left\{\tilde{\kappa} \in \mathfrak{K} ; U_{\tilde{\kappa}} \cap U_{\kappa} \neq \emptyset\right\}
$$


has cardinality $\leq k$ for each $\kappa \in \mathfrak{K}$. An atlas is shrinkable if it consists of normalized charts and there exists $r \in(0,1)$ such that $\left\{\kappa^{-1}\left(r \kappa\left(U_{\kappa}\right)\right) ; \kappa \in \mathfrak{K}\right\}$ is a cover of $M$.

\section{$(M, g)$ is a uniformly regular Riemannian manifold if}

(i) it possesses a shrinkable atlas $\mathfrak{K}$ of finite multiplicity which is orientation preserving if $M$ is oriented.

(ii) $\left\|\tilde{\kappa} \circ \kappa^{-1}\right\|_{k, \infty} \leq c(k), \kappa, \tilde{\kappa} \in \mathfrak{K}, k \in \mathbb{N}$.

(iii) $\kappa_{*} g \sim g_{m}, \kappa \in \mathfrak{K}$.

(iv) $\left\|\kappa_{*} g\right\|_{k, \infty} \leq c(k), \kappa \in \mathfrak{K}, k \in \mathbb{N}$.

In (ii) and in similar situations it is understood that only $\kappa, \tilde{\kappa} \in \mathfrak{K}$ with $U_{\kappa} \cap U_{\tilde{\kappa}} \neq \emptyset$ are being considered. Here and below, we employ the standard definitions of push-forward and pull-back operators. An atlas satisfying (9.1) (i) and (ii) is called uniformly regular. Henceforth, it is assumed that

- $(M, g)$ is a uniformly regular Riemannian manifold without boundary and $\mathfrak{K}$ is an atlas possessing properties (9.1).

Observe that $\mathfrak{K}$ is countable. A localization system for $M$ subordinate to $\mathfrak{K}$ is a family $\left\{\left(\pi_{\kappa}, \chi_{\kappa}\right) ; \kappa \in \mathfrak{K}\right\}$ such that

(i) $\pi_{\kappa} \in \mathcal{D}\left(U_{\kappa},[0,1]\right)$ and $\left\{\pi_{\kappa}^{2} ; \kappa \in \mathfrak{K}\right\}$ is a partition of unity on $M$ subordinate to the covering $\left\{U_{\kappa} ; \kappa \in \mathfrak{K}\right\}$.

(ii) $\chi_{\kappa}=\kappa^{*} \chi$ with $\chi \in \mathcal{D}\left(Q^{m},[0,1]\right)$

$$
\text { and } \chi \mid \operatorname{supp}\left(\kappa_{*} \pi_{\kappa}\right)=\mathbf{1} \text { for } \kappa \in \mathfrak{K} \text {. }
$$

(iii) $\left\|\kappa_{*} \pi_{\kappa}\right\|_{k, \infty}+\left\|\kappa_{*} \chi_{\kappa}\right\|_{k, \infty} \leq c(k), \kappa \in \mathfrak{K}, k \in \mathbb{N}$.

Lemma 3.2 of [8] guarantees the existence of such systems.

Using $T_{p} Q^{m}=\mathbb{R}^{m}$ for $p \in Q^{m}$ we get

$$
T_{\tau}^{\sigma} Q^{m} \otimes F=Q^{m} \times\left(\left(\mathbb{R}^{m}\right)^{\otimes \sigma} \otimes\left(\mathbb{R}^{m}\right)^{* \otimes \tau} \otimes F\right) .
$$

Of course, we identify $\left(\mathbb{R}^{m}\right)^{*}$ canonically with $\mathbb{R}^{m}$, but for clarity we continue to denote it by $\left(\mathbb{R}^{m}\right)^{*}$. We endow $T_{\tau}^{\sigma} Q^{m} \otimes F$ with the inner product

$$
(\cdot \mid \cdot)_{T_{\tau}^{\sigma} Q^{m} \otimes F}:=(\cdot \mid \cdot)^{\otimes \sigma} \otimes(\cdot \mid \cdot)^{\otimes \tau} \otimes(\cdot \mid \cdot)_{F} \cdot
$$

The standard basis $\left(e_{1}, \ldots, e_{m}\right)$ of $\mathbb{R}^{m}$ and its dual basis $\left(\varepsilon^{1}, \ldots, \varepsilon^{m}\right)$ of $\left(\mathbb{R}^{m}\right)^{*}$ induce the coordinate frame

$$
\left\{e_{(i)} \otimes \varepsilon^{(j)} ;(i) \in \mathbb{J}_{\sigma},(j) \in \mathbb{J}_{\tau}\right\}
$$

on $T_{\tau}^{\sigma} Q^{m}$, where $e_{(i)}:=e_{i_{1}} \otimes \cdots \otimes e_{i_{\sigma}}$, etc. Then

$$
u \in\left(T_{\tau}^{\sigma} Q^{m} \otimes F\right)_{p}=\mathcal{L}\left(\left(\left(\mathbb{R}^{m}\right)^{*}\right)^{\otimes \sigma} \otimes\left(\mathbb{R}^{m}\right)^{\otimes \tau}, F\right)
$$

has the matrix representation $\left[u_{(j)}^{(i)}\right] \in F^{m^{\sigma} \times m^{\tau}}$. If $n=0$, then $F=\mathbb{R}$. We endow $F^{m^{\sigma} \times m^{\tau}}$ with the inner product

$$
\left(\left[u_{(j)}^{(i)}\right] \mid\left[v_{(\tilde{\jmath})}^{(\tilde{\tau})}\right]\right)_{\mathrm{HS}, F}:=\sum_{(i) \in \mathbb{J}_{\sigma},(j) \in \mathbb{J}_{\tau}}\left(u_{(j)}^{(i)} \mid v_{(j)}^{(i)}\right)_{F} .
$$


It coincides with the Hilbert-Schmidt inner product if $F=\mathbb{R}$. From now on, by $E$ we always mean $\left(E,(\cdot \mid \cdot)_{E}\right)$, where

$$
\text { - } E=E_{\tau}^{\sigma}=E_{\tau}^{\sigma}(F):=F^{m^{\sigma} \times m^{\tau}}, \quad(\cdot \mid \cdot)_{E}:=(\cdot \mid \cdot)_{\mathrm{HS}, F} .
$$

It follows from $(9.3)$ that $u \mapsto\left[u_{(j)}^{(i)}\right]$ defines an isometric isomorphism by which

$$
\text { we identify } T_{\tau}^{\sigma} Q^{m} \otimes F \text { with } Q^{m} \times E \text {. }
$$

Given Banach spaces $X_{0}, X_{1}$ and $j \in \dot{\mathbb{N}}$, we denote by $\mathcal{L}^{j}\left(X_{0} ; X_{1}\right)$ the Banach space of all $j$-linear maps from $X_{0} \times \cdots \times X_{0}$ ( $j$ copies) into $X_{1}$, and $\mathcal{L}^{0}\left(X_{0} ; X_{1}\right):=X_{1}$.

Suppose $v$ is a $C^{j}$-section of $Q^{m} \times E$, that is, $v \in C^{j}\left(Q^{m}, E\right)$. Then

$$
\partial^{j} v \in C\left(Q^{m}, \mathcal{L}^{j}\left(\mathbb{R}^{m}, E\right)\right)=C\left(Q^{m}, E_{\tau+j}^{\sigma}\right),
$$

using canonical identifications.

Let $\kappa \in \mathfrak{K}$. Suppose $u \in C(V)$. Denote by $\left[u_{(k)}^{(j)}\right]$ the representation of $u$ on $U_{\kappa}$ with respect to the coordinate frame (1.2). Then

$$
\kappa_{*} u:=\left[\kappa_{*} u_{(k)}^{(j)}\right]=\left[u_{(k)}^{(j)} \circ \kappa^{-1}\right] \in C\left(Q^{m}, E\right) .
$$

The push-forward of $\nabla^{j}: C^{j}(V) \rightarrow C\left(V_{\tau+j}^{\sigma}\right)$ is defined by

$$
\left(\kappa_{*} \nabla^{j}\right) v:=\kappa_{*}\left(\nabla^{j}\left(\kappa^{*} v\right)\right), \quad v \in C^{j}\left(Q^{m}, E\right) .
$$

Then $\kappa_{*} \nabla$ is a metric connection on $Q^{m} \times E$ which satisfies

$$
\kappa_{*} \nabla^{j} v=\partial^{j} v+\sum_{i=0}^{j-1} b_{j, i}^{\kappa} \partial^{i} v, \quad v \in C^{j}\left(Q^{m}, E\right),
$$

with $b_{j, i}^{\kappa} \in C^{\infty}\left(Q^{m}, \mathcal{L}\left(E_{\tau+i}^{\sigma}, E_{\tau+j}^{\sigma}\right)\right.$ and

$$
\left\|b_{j, i}^{\kappa}\right\|_{k, \infty} \leq c(j, k), \quad 0 \leq i \leq j-1, \quad j, k \in \mathbb{N}, \quad \kappa \in \mathfrak{K},
$$

(see the proof of [8, Lemma 3.1]).

Considering $\mathfrak{K}$ as an index set endowed with the discrete topology, we set $\mathfrak{F}_{q}^{s}:=C\left(\mathfrak{K}, \mathfrak{F}_{q}^{s}\right)$, the space of all 'sequences' in $\mathfrak{F}_{q}^{s}$ 'enumerated' by $\mathfrak{K}$, and

$$
\ell_{q}\left(\mathfrak{F}_{q}^{s}\right):=\ell_{q}\left(\mathfrak{K}, \mathfrak{F}_{q}^{s}\right), \quad 1 \leq q \leq \infty .
$$

If $k<s<k+1$ with $k \in \mathbb{N}$, then $\ell_{\infty \text {,unif }}\left(b u c^{s}\right)$ is the closed linear subspace of $\ell_{\infty}\left(\mathfrak{F}_{\infty}^{s}\right)$ of all $\boldsymbol{v}=\left(v_{\kappa}\right)$ such that $\lim _{\delta \rightarrow 0} \max _{|\alpha|=k}\left[\partial^{\alpha} v_{\kappa}\right]_{s-k, \infty}^{\delta}=0$, uniformly with respect to $\kappa \in \mathfrak{K}$.

Now we fix a localization system for $M$. Then we define

$$
\mathcal{R} u:=\sum_{\kappa} \pi_{\kappa} \kappa^{*} u_{\kappa}, \quad \mathcal{R}^{c} u:=\left(\kappa_{*}\left(\pi_{\kappa} u\right)\right)_{\kappa \in \mathfrak{K}}
$$

for $\boldsymbol{u}=\left(u_{\kappa}\right) \in \mathfrak{F}_{q}^{s}$ and $u \in L_{1, \text { loc }}\left(V_{\tau}^{\sigma}\right)$, whenever the series is absolutely convergent. In the following, we often identify functions with multiplication operators. 
Theorem 9.1. Suppose $s \in \mathbb{R}^{+}$and $1 \leq q<\infty$. Then $\left(\mathcal{R}, \mathcal{R}^{c}\right)$ is an $r$-e pair for

$$
\left(\ell_{q}\left(W_{q}^{s}\right), W_{q}^{s}(V)\right),\left(\ell_{\infty}\left(B U C^{s}\right), B C^{s}(V)\right), \text { and }\left(\ell_{\infty, \text { unif }}\left(b u c^{s}\right), b c^{s}(V)\right),
$$

provided $s \notin \mathbb{N}$ in the last instance.

Proof. [8, Theorem 6.1] and [7, Theorem 12.5].

The next theorem shows that, similarly as in the compact case, general uniformly regular Riemannian manifolds can be characterized by means of local coordinates.

Theorem 9.2. Suppose $s \in \mathbb{R}^{+}$and $1 \leq q<\infty$. Then

$$
u \mapsto\left(\sum_{\kappa \in \mathfrak{K}}\left\|\kappa_{*} u\right\|_{W_{q}^{s}\left(Q^{m}, E\right)}^{q}\right)^{1 / q}
$$

is a norm for $W_{q}^{s}(V)$, and

$$
u \mapsto \sup _{\kappa \in \mathfrak{K}}\left\|\kappa_{*} u\right\|_{B C^{s}\left(Q^{m}, E\right)}
$$

is one for $B C^{s}(V)$.

If $k<s<k+1$ with $k \in \mathbb{N}$, then $u \in b u c^{s}(V)$ iff $u \in B C^{k}(V)$ and

$$
\lim _{\delta \rightarrow 0}\left[\partial^{\alpha}\left(\kappa_{*} u\right)\right]_{s-k, \infty}^{\delta}=0, \quad \alpha \in \mathbb{N}^{m}, \quad|\alpha|=k,
$$

uniformly with respect to $\kappa \in \mathfrak{K}$.

Proof. (1) We set $S_{\kappa \tilde{\kappa}}:=\kappa_{*} \circ \tilde{\kappa}^{*} \circ \chi$ for $\kappa, \tilde{\kappa} \in \mathfrak{K}$. If $s \in \mathbb{N}$, then it is a consequence of (9.1) (ii) and the chain rule that

$$
S_{\kappa \tilde{\kappa}} \in \mathcal{L}\left(W_{q}^{s}\right) \cap \mathcal{L}\left(B C^{s}\right),
$$

uniformly with respect to $\kappa \in \mathfrak{K}$ and $\tilde{\kappa} \in \mathfrak{N}(\kappa)$. From this we obtain (9.6) for $s \notin \mathbb{N}$ by interpolation with the real interpolation functor $(\cdot, \cdot)_{\theta, q}$, respectively $(\cdot, \cdot)_{\theta, \infty}$ in the case of $B C$ spaces.

Since $\left\|\tilde{\kappa} \circ \kappa^{-1}\right\|_{k+1, \infty} \leq c(k)$ for $\kappa \in \mathfrak{K}$ and $\tilde{\kappa} \in \mathfrak{N}(\kappa)$, the mean-value theorem implies that $\partial^{k}\left(\tilde{\kappa} \circ \kappa^{-1}\right)$ is uniformly Lipschitz continuous, uniformly with respect $\kappa \in \mathfrak{K}$ and $\tilde{\kappa} \in \mathfrak{N}(\kappa)$. From this we get $S_{\kappa \tilde{\kappa}} \in \mathcal{L}\left(B U C^{k}\right)$ for $k \in \mathbb{N}$, uniformly with respect to $\kappa \in \mathfrak{K}$ and $\tilde{\kappa} \in \mathfrak{N}(\kappa)$. Now, given $s \in \mathbb{R}^{+} \backslash \mathbb{N}$, we deduce by continuous interpolation

$$
S_{\kappa \tilde{\kappa}} \in \mathcal{L}\left(b u c^{s}\right),
$$

uniformly with respect $\kappa \in \mathfrak{K}$ and $\tilde{\kappa} \in \mathfrak{N}(\kappa)$.

(2) Using $\sum_{\kappa} \pi_{\kappa}^{2}=1$ we find, due to $\chi_{\kappa} \pi_{\kappa}=\pi_{\kappa}$,

$$
\kappa_{*} u=\sum_{\tilde{\kappa}} \kappa_{*}\left(\pi_{\tilde{\kappa}}^{2} u\right)=\sum_{\tilde{\kappa} \in \mathfrak{N}(\kappa)}\left(\kappa_{*} \pi_{\tilde{\kappa}}\right) S_{\kappa \tilde{\kappa}}\left(\tilde{\kappa}_{*}\left(\pi_{\tilde{\kappa}} u\right)\right)
$$

for $u \in C(V)$ and $\kappa \in \mathfrak{K}$. Observing $\kappa_{*} \pi_{\tilde{\kappa}}=S_{\kappa \tilde{\kappa}}\left(\tilde{\kappa}_{*} \pi_{\tilde{\kappa}}\right)$, we infer from (9.2) (iii) and step (1)

$$
\left\|\kappa_{*} \pi_{\tilde{\kappa}}\right\|_{\ell, \infty} \leq c(\ell), \quad \kappa \in \mathfrak{K}, \quad \tilde{\kappa} \in \mathfrak{N}(\kappa), \quad \ell \in \mathbb{N} .
$$


From this, (9.6), (9.8), and Theorem 9.1 it follows

$$
\left(\sum_{\kappa}\left\|\kappa_{*} u\right\|_{W_{q}^{s}\left(Q^{m}, E\right)}^{q}\right)^{1 / q} \leq c\left\|\mathcal{R}^{c} u\right\|_{\ell_{q}\left(W_{p}^{s}\right)}
$$

and

$$
\sup _{\kappa}\left\|\kappa_{*} u\right\|_{B C^{s}\left(Q^{m}, E\right)} \leq c\|\mathcal{R} u\|_{\ell_{\infty}\left(B C^{s}\right)}
$$

On the other hand, $\kappa_{*}\left(\pi_{\kappa} u\right)=\left(\kappa_{*} \pi_{\kappa}\right) \kappa_{*} u$ and (9.2) (iii) imply

$$
\left\|\kappa_{*}\left(\pi_{\kappa} u\right)\right\|_{W_{q}^{s}\left(\mathbb{R}^{m}, E\right)} \leq c(k)\left\|\kappa_{*} u\right\|_{W_{q}^{s}\left(Q^{m}, E\right)}
$$

and

$$
\left\|\kappa_{*}\left(\pi_{\kappa} u\right)\right\|_{B C^{s}\left(\mathbb{R}^{m}, E\right)} \leq c(k)\left\|\kappa_{*} u\right\|_{B C^{s}\left(Q^{m}, E\right)}
$$

for $\kappa \in \mathfrak{K}, \quad k \in \mathbb{N}$, and $0 \leq s \leq k$. Consequently, the left-hand sides of (9.9) and (9.10) can be bounded from below by $c^{-1}\left\|\mathcal{R}^{c} u\right\|_{\ell_{q}\left(W_{q}^{s}\right)}$, respectively by $c^{-1}\left\|\mathcal{R}^{c} u\right\|_{\ell_{\infty}\left(B C^{s}\right)}$.

It follows from Theorem 9.1 and general properties of retractions and coretractions (e.g., (7.8) and (7.9) in [7]) that $u \mapsto\left\|\mathcal{R}^{c} u\right\|_{\ell_{q}\left(W_{q}^{s}\right)}$ is an equivalent norm for $W_{q}^{s}(V)$ and $u \mapsto\left\|\mathcal{R}^{c} u\right\|_{\ell_{\infty}\left(B C^{s}\right)}$ is one for $B C^{s}(V)$. This implies the first part of the assertion. The last one is now a consequence of (9.8), (9.7), and Theorem 9.1.

\section{Localizations of Elliptic Operators}

Unless explicitly stated otherwise, it is assumed that

$$
\begin{array}{ll}
\text { (i) } & 0<\bar{s}<1 \\
\text { (ii) } & \mathcal{A}=\sum_{j=0}^{r} a_{r} \cdot \nabla^{r} \text { is } \bar{s} \text {-regular and } \\
& \text { uniformly normally } \varepsilon \text {-elliptic on }(M, g) . \\
\text { (iii) } & 0 \leq s \leq \bar{s} \text { and } s<\bar{s} \text { if } q<\infty .
\end{array}
$$

Thus we consider low-regularity autonomous problems. We also suppose

- $(s, q)$ is 1 -admissible.

For $\kappa \in \mathfrak{K}$ we define $\kappa_{*} \mathcal{A}$ by $\left(\kappa_{*} \mathcal{A}\right) v=\kappa_{*}\left(\mathcal{A}\left(\kappa^{*} v\right)\right)$ for $v \in C^{r}\left(Q^{m}, E\right)$. Then

$$
\kappa_{*} \mathcal{A}=\sum_{j=0}^{r}\left(\kappa_{*} a_{j}\right) \cdot \kappa_{*} \nabla^{j}
$$

It follows from Theorem 9.2 that, setting $\mathcal{L}^{j}:=\mathcal{L}^{j}\left(\mathbb{R}^{m}, E\right)$,

$$
\left(\kappa_{*} a_{j}\right)_{\kappa \in \mathfrak{K}} \in \ell_{\infty, \text { unif }}\left(b u c^{\bar{s}}\left(Q^{m}, \mathcal{L}^{j}\right)\right), \quad 0 \leq j \leq r .
$$

Note that

$$
\mathfrak{s}\left(\kappa_{*} \mathcal{A}\right)(\cdot, \xi)=\kappa_{*}\left(\mathfrak{s} \mathcal{A}\left(\cdot, \kappa^{*} \xi\right)\right), \quad \xi \in \mathbb{R}^{m} .
$$

It is a consequence of (9.1) that $\left|\kappa^{*} \xi\right|_{1}^{0} \sim \kappa^{*}|\xi|$ for $\xi \in \Gamma\left(T^{*} Q^{m}\right)$ and $\kappa \in \mathfrak{K}$ (cf. [8, Lemma 3.1]). From this, (10.3),

$$
\kappa_{*}\left(\mathfrak{s} \mathcal{A}\left(\cdot, \kappa^{*} \xi\right)\right)=\kappa_{*}\left(\left(\left|\kappa^{*} \xi\right|_{1}^{0}\right)^{r} \mathfrak{s} \mathcal{A}\left(\cdot, \kappa^{*} \xi /\left|\kappa^{*} \xi\right|_{1}^{0}\right)\right),
$$


and the uniform normal $\varepsilon$-ellipticity of $\mathcal{A}$ we deduce the existence of a constant $c \geq 1$ such that, setting $\varepsilon_{1}:=\varepsilon / c$,

$\kappa_{*} \mathcal{A}$ is uniformly normally $\varepsilon_{1}$-elliptic on $\left(Q^{m}, g_{m}\right)$, uniformly with respect to $\kappa \in \mathfrak{K}$.

For $\delta>0$ we denote by $h_{\delta}: \mathbb{R}^{m} \rightarrow \delta Q^{m}$ the radial retraction. Thus $h_{\delta}(x)=x$ if $x \in \delta Q^{m}$, and $h_{\delta}(x)=\delta x /|x|_{\infty}$ otherwise. Note that $h_{\delta}$ is uniformly Lipschitz continuous with Lipschitz constant 2 (cf. [2, Lemma 19.8]). We set

$$
a_{\kappa}:=\left(\kappa_{*} a_{r}\right) \circ h_{1}, \quad \kappa \in \mathfrak{K} .
$$

Then

$$
a_{\kappa}(x)=\kappa_{*} a_{r}(x), \quad x \in Q^{m},
$$

and

$$
a_{\kappa} \in \operatorname{buc} \bar{s}^{\bar{s}}\left(\mathbb{R}^{m}, \mathcal{L}^{r}\right), \quad\left\|a_{\kappa}\right\|_{\bar{s}, \infty} \leq 2\left\|a_{r}\right\|_{\bar{s}, \infty}, \quad \kappa \in \mathfrak{K} .
$$

These estimates, (10.2), and (10.4) imply

$$
\left(a_{\kappa}\right) \in \ell_{\infty, \text { unif }}\left(b u c^{\bar{s}}\left(\mathbb{R}^{m}, \mathcal{L}^{r}\right)\right)
$$

and

$$
a_{\kappa} \cdot \partial^{r} \text { is uniformly normally } \varepsilon_{1} \text {-elliptic on }\left(\mathbb{R}^{m}, g_{m}\right) \text {, }
$$

uniformly with respect to $\kappa \in \mathfrak{K}$.

For each $\alpha$ in a countable index set $A$ let $E_{\alpha}$ and $F_{\alpha}$ be Banach spaces. Then $\mathcal{L}(\boldsymbol{E}, \boldsymbol{F}):=\prod_{\alpha} \prod_{\beta} \mathcal{L}\left(E_{\beta}, F_{\alpha}\right)$. Using obvious matrix notation, we define a linear map $\boldsymbol{A}: \boldsymbol{E} \rightarrow \boldsymbol{F}$ by

$$
(\boldsymbol{A u})_{\alpha}:=\sum_{\beta} A_{\alpha \beta} u_{\beta}, \quad \alpha \in \mathrm{A}, \quad\left[A_{\alpha \beta}\right] \in \mathcal{L}(\boldsymbol{E}, \boldsymbol{F}), \quad \boldsymbol{u}=\left(u_{\beta}\right) \in \boldsymbol{E},
$$

whenever these series converge absolutely in $E_{\alpha}$. We often identify $\left[A_{\alpha \beta}\right]$ with $\boldsymbol{A}$. Furthermore,

$$
\operatorname{diag}\left[A_{\alpha}\right]:=\left[A_{\alpha} \delta_{\alpha \beta}\right] \in \mathcal{L}(\boldsymbol{E}, \boldsymbol{F}), \quad A_{\alpha} \in \mathcal{L}\left(E_{\alpha}, F_{\alpha}\right),
$$

where $\delta_{\alpha \beta}$ is the Kronecker symbol.

We fix $q \in[1, \infty]$ and set

$$
\mathbb{E}^{s}:=\left\{\begin{array}{lr}
\ell_{q}\left(\mathfrak{F}_{q}^{s}\right), & 1 \leq q<\infty \\
\ell_{\infty, \text { unif }}\left(\mathfrak{F}_{\infty}^{s}\right), & q=\infty
\end{array}\right.
$$

It follows from (10.7) that

$$
A:=\operatorname{diag}\left[A_{\kappa}\right]:=\operatorname{diag}\left[a_{\kappa} \cdot \partial^{r}\right] \in \mathcal{L}\left(\mathbb{E}^{s+r}, \mathbb{E}^{s}\right) .
$$

Lemma 10.1. There exist

$$
B, B^{\prime} \in \mathcal{L}\left(\mathbb{E}^{s+r-1}, \mathbb{E}^{s}\right)
$$

such that

$$
\mathcal{A} \circ \mathcal{R}=\mathcal{R} \circ(A+B), \quad \mathcal{R}^{c} \circ \mathcal{A}=\left(A+B^{\prime}\right) \circ \mathcal{R}^{c} .
$$


Proof. (1) We set $\nabla_{\kappa}:=\kappa_{*} \nabla$ and denote by $[\cdot, \cdot]$ commutators. Then, given $u_{\kappa} \in \mathfrak{F}_{q}^{s+r}$,

$$
a_{j} \cdot \nabla^{j}\left(\pi_{\kappa} \kappa^{*} u_{\kappa}\right)=\pi_{\kappa} \kappa^{*}\left(\kappa_{*} a_{j} \cdot \nabla_{\kappa}^{j} u_{\kappa}\right)+a_{j} \cdot\left[\nabla^{j}, \pi_{\kappa}\right] \kappa^{*} u_{\kappa} .
$$

We multiply the last term with $1=\sum_{\tilde{\kappa}} \pi_{\tilde{\kappa}}^{2}$ and use $\kappa^{*} u_{\kappa}=\tilde{\kappa}^{*}\left(S_{\tilde{\kappa} \kappa} u_{\kappa}\right)$. Then it takes the form

$$
\sum_{\tilde{\kappa} \in \mathfrak{N}(\kappa)} \pi_{\tilde{\kappa}} \tilde{\kappa}^{*}\left(\left(\tilde{\kappa}_{*} \pi_{\tilde{\kappa}}\right) \tilde{\kappa}_{*} a_{j} \cdot\left[\nabla_{\tilde{\kappa}}^{j}, \tilde{\kappa}_{*} \pi_{\kappa}\right] S_{\tilde{\kappa} \kappa} u_{\kappa}\right) .
$$

Note that $\operatorname{supp}\left(\kappa_{*} \pi_{\kappa}\right) \subset \chi^{-1}(1)$ and (10.6) imply

$$
\begin{aligned}
\pi_{\kappa} \kappa^{*}\left(\kappa_{*} a_{r} \cdot \nabla_{\kappa}^{r} u_{\kappa}\right) & =\kappa^{*}\left(\left(\kappa_{*} \pi_{\kappa}\right) \kappa_{*} a_{r} \cdot \nabla_{\kappa}^{r} u_{\kappa}\right) \\
& =\kappa^{*}\left(\left(\kappa_{*} \pi_{\kappa}\right) a_{\kappa} \cdot \nabla_{\kappa}^{r} u_{\kappa}\right) \\
& =\pi_{\kappa} \kappa^{*}\left(a_{\kappa} \cdot \partial^{r} u_{\kappa}\right)+\pi_{\kappa} \kappa^{*}\left(a_{\kappa} \cdot\left(\nabla_{\kappa}^{r}-\partial^{r}\right) u_{\kappa}\right) \\
& =\pi_{\kappa} \kappa^{*}\left(A_{\kappa} u_{\kappa}\right)+\pi_{\kappa} \kappa^{*}\left(a_{\kappa} \cdot\left(\nabla_{\kappa}^{r}-\partial^{r}\right) u_{\kappa}\right) .
\end{aligned}
$$

We put

$$
\begin{aligned}
B_{\tilde{\kappa} \kappa} u_{\kappa}:= & \delta_{\tilde{\kappa} \kappa}\left(a_{\kappa} \cdot\left(\nabla_{\kappa}^{r}-\partial^{r}\right) u_{\kappa}+\sum_{j=0}^{r-1} \kappa_{*} a_{j} \cdot \nabla_{\kappa}^{j} u_{\kappa}\right) \\
& +\sum_{j=0}^{r}\left(\tilde{\kappa}_{*} \pi_{\tilde{\kappa}}\right) \tilde{\kappa}_{*} a_{j} \cdot\left[\nabla_{\tilde{\kappa}}^{j}, \tilde{\kappa}_{*} \pi_{\kappa}\right] S_{\tilde{\kappa} \kappa} u_{\kappa}
\end{aligned}
$$

for $\kappa \in \mathfrak{K}$ and $\tilde{\kappa} \in \mathfrak{N}(\kappa)$, and $B_{\tilde{\kappa} \kappa}:=0$ if $\tilde{\kappa} \notin \mathfrak{N}(\kappa)$. It follows from (10.2), (10.7), (9.5), (9.6), (9.7), and (9.2) that

$$
B_{\tilde{\kappa} \kappa} \in \mathcal{L}\left(\mathfrak{F}_{q}^{s+r-1}, \mathfrak{F}_{q}^{s}\right), \quad\left\|B_{\tilde{\kappa} \kappa}\right\| \leq c, \quad \kappa, \tilde{\kappa} \in \mathfrak{K} .
$$

From (10.12)-(10.14) we get, due to (9.2),

$$
\mathcal{A}\left(\pi_{\kappa} \kappa^{*} u_{\kappa}\right)=\pi_{\kappa} \kappa^{*}\left(A_{\kappa} u_{\kappa}\right)+\sum_{\tilde{\kappa} \in \mathfrak{N}(\kappa)} \pi_{\tilde{\kappa}} \tilde{\kappa}_{*}\left(B_{\tilde{\kappa} \kappa} u_{\kappa}\right), \quad \kappa \in \mathfrak{K} .
$$

Now we sum over $\kappa \in \mathfrak{K}$ and interchange the order of summation in the resulting double sum. Then we obtain

$$
\mathcal{A}(\mathcal{R} \boldsymbol{u})=\mathcal{R}(A \boldsymbol{u})+\mathcal{R}\left(\left(\sum_{\tilde{\kappa}} B_{\kappa \tilde{\kappa}} u_{\tilde{\kappa}}\right)_{\kappa \in \mathfrak{K}}\right), \quad \boldsymbol{u}=\left(u_{\kappa}\right) \in \mathbb{E}^{s+r} .
$$

We set $B:=\left[B_{\kappa \tilde{\kappa}}\right]$. Let $k \in \mathbb{N}$ be such that $\operatorname{card}(\mathfrak{N}(\kappa)) \leq k$ for $\kappa \in \mathfrak{K}$. Then $\left[B_{\kappa \tilde{\kappa}}\right]$ has for each $\kappa \in \mathfrak{K}$ at most $k$ non-zero off-diagonal elements. From this and (10.15) it follows that

$$
B \in \mathcal{L}\left(\ell_{q}\left(\mathfrak{F}_{q}^{s+r-1}\right), \ell_{q}\left(\mathfrak{F}_{q}^{s}\right)\right) .
$$

If $\boldsymbol{u} \in \ell_{\infty, \text { unif }}\left(\mathfrak{F}_{\infty}^{s}\right)$, then it is verified that $B \boldsymbol{u}$ belongs to the same space. This proves (10.10) for $B$. The first relation of (10.11) follows from (10.16).

(2) Similarly as above,

$$
\kappa_{*}\left(\pi_{\kappa} a_{j} \cdot \nabla^{j} u\right)=\kappa_{*} a_{j} \cdot \nabla_{\kappa}^{j}\left(\kappa_{*}\left(\pi_{\kappa} u\right)\right)-\kappa_{*} a_{j} \cdot\left[\nabla_{\kappa}^{j}, \kappa_{*} \pi_{\kappa}\right] \kappa_{*} u .
$$


Due to (9.8), the last term can be rewritten as

$$
-\sum_{\tilde{\kappa} \in \mathfrak{N}(\kappa)} \kappa_{*} a_{j} \cdot\left[\nabla_{\kappa}^{j}, \kappa_{*} \pi_{\kappa}\right]\left(\kappa_{*} \pi_{\tilde{\kappa}}\right) S_{\kappa \tilde{\kappa}}\left(\tilde{\kappa}_{*}\left(\pi_{\tilde{\kappa}} u\right)\right) .
$$

We put, for $u_{\tilde{\kappa}} \in \mathfrak{F}_{q}^{s+r-1}$,

$$
\begin{aligned}
B_{\kappa \tilde{\kappa}}^{\prime} u_{\tilde{\kappa}}:= & \delta_{\kappa \tilde{\kappa}}\left(a_{\kappa} \cdot\left(\nabla_{\kappa}^{r}-\partial^{r}\right) u_{\tilde{\kappa}}+\sum_{j=0}^{r-1} \kappa_{*} a_{j} \cdot \nabla_{\kappa}^{j} u_{\tilde{\kappa}}\right) \\
& -\kappa_{*} a_{j} \cdot\left[\nabla_{\kappa}^{j}, \kappa_{*} \pi_{\kappa}\right]\left(\kappa_{*} \pi_{\tilde{\kappa}}\right) S_{\kappa \tilde{\kappa}} u_{\tilde{\kappa}}
\end{aligned}
$$

if $\kappa \in \mathfrak{K}$ and $\tilde{\kappa} \in \mathfrak{N}(\kappa)$, and $B_{\kappa \tilde{\kappa}}^{\prime} u_{\tilde{\kappa}}:=0$ if $\tilde{\kappa} \notin \mathfrak{N}(\kappa)$. Then $B^{\prime}:=\left[B_{\kappa \tilde{\kappa}}^{\prime}\right]$ satisfies (10.10). Furthermore, (10.17)-(10.19) and (10.14) imply

$$
\kappa_{*}\left(\pi_{\kappa} \mathcal{A} u\right)=A_{\kappa}\left(\kappa_{*}\left(\pi_{\kappa} u\right)\right)+\sum_{\tilde{\kappa}} B_{\kappa \tilde{\kappa}}^{\prime}\left(\tilde{\kappa}_{*}\left(\pi_{\tilde{\kappa}} u\right)\right), \quad \kappa \in \mathfrak{K} .
$$

This shows that the second relation of (10.11) is also satisfied.

Corollary 10.2. Suppose $0 \in \rho(A+B) \cap \rho\left(A+B^{\prime}\right)$. Then $0 \in \rho(\mathcal{A})$ and

$$
\mathcal{A}^{-1}=\mathcal{R} \circ(A+B)^{-1} \circ \mathcal{R}^{c} .
$$

Proof. Let $u \in \mathfrak{F}_{q}^{s+r}(V)$ satisfy $\mathcal{A} u=0$. Then (10.11) implies

$$
0=\mathcal{R}^{c} \mathcal{A} u=\left(A+B^{\prime}\right) \mathcal{R}^{c} u=0 .
$$

Hence $\mathcal{R}^{c} u=0$ and, thus, $u=\mathcal{R} \mathcal{R}^{c} u=0$. Consequently, $\mathcal{A}$ is injective.

Suppose $f \in \mathfrak{F}_{q}^{s}(V)$. There is a unique $\boldsymbol{u} \in \mathbb{E}_{q}^{s+r}$ with $(A+B) \boldsymbol{u}=\mathcal{R}^{c} f$. Setting

$$
u:=\mathcal{R} u=\mathcal{R}(A+B)^{-1} \mathcal{R}^{c} f \in \mathfrak{F}_{q}^{s+r}(V),
$$

we get

$$
\mathcal{A} u=\mathcal{A R}(A+B)^{-1} \mathcal{R}^{c} f=\mathcal{R}(A+B)(A+B)^{-1} \mathcal{R}^{c} f=\mathcal{R R}^{c} f=f
$$

by (10.11). Thus $\mathcal{A}$ is surjective and (10.20) applies. Since $\mathcal{A}$ is closed, when considered as a linear operator in $\mathfrak{F}_{q}^{s}(V)$, we get $0 \in \rho(\mathcal{A})$.

\section{Localizations of Parabolic Operators}

We require again assumption (10.1) and assume that $(s, q)$ is $r$-admissible. Then

and

$$
\mathfrak{F}_{q}^{s / \vec{r}}:=C\left(\mathfrak{K}, \mathfrak{F}_{q}^{s / \vec{r}}(\mathbb{H}, E)\right)
$$

$$
\mathbb{E}^{s / \vec{r}}:=\left\{\begin{array}{lr}
\ell_{q}\left(\mathfrak{F}_{q}^{s / \vec{r}}(\mathbb{H}, E)\right), & 1 \leq q<\infty, \\
\ell_{\infty, \text { unif }}\left(\mathfrak{F}_{\infty}^{s / \vec{r}}(\mathbb{H}, E)\right), & q=\infty .
\end{array}\right.
$$

We denote the point-wise extension of $\left(\mathcal{R}, \mathcal{R}^{c}\right)$ to $t$-dependent functions again by the same symbol. It is easy to extend Theorem 9.1 to obtain the following analogue.

Theorem 11.1. $\left(\mathcal{R}, \mathcal{R}^{c}\right)$ is an r-e pair for $\left(\mathbb{E}^{s / \vec{r}}, \mathfrak{F}_{q}^{s / \vec{r}}\left(V \times \mathbb{R}^{+}\right)\right)$. 
Let the hypotheses of Lemma 10.1 be satisfied. We set $\partial_{t, \kappa}:=\partial_{t}$ for $\kappa \in \mathfrak{K}$ and $\partial_{t}:=\operatorname{diag}\left[\partial_{t, \kappa}\right]$. We write $\gamma_{\kappa}:=\gamma_{\partial \mathbb{H}}$ for $\kappa \in \mathfrak{K}$, where $\gamma_{\mathbb{H}}$ is the trace operator on $\partial \mathbb{H}$, and $\boldsymbol{\gamma}:=\operatorname{diag}\left[\gamma_{\kappa}\right]$.

The next lemma and its corollary are obvious consequences of the results of the preceding section.

Lemma 11.2. It holds

$$
\begin{aligned}
\left(\partial_{t}+\mathcal{A}\right) \circ \mathcal{R} & =\mathcal{R} \circ\left(\partial_{t}+A+B\right), \\
\mathcal{R}^{c}\left(\partial_{t}+\mathcal{A}\right) & =\left(\partial_{t}+A+B^{\prime}\right) \circ \mathcal{R}^{c},
\end{aligned}
$$

and

$$
\gamma \circ \mathcal{R}=\mathcal{R} \circ \boldsymbol{\gamma}, \quad \mathcal{R}^{c} \circ \gamma=\boldsymbol{\gamma} \circ \mathcal{R}^{c}
$$

Corollary 11.3. Suppose

$$
\begin{aligned}
& \left(\boldsymbol{\partial}_{t}+A+B, \boldsymbol{\gamma}\right) \text { and }\left(\boldsymbol{\partial}_{t}+A+B^{\prime}, \boldsymbol{\gamma}\right) \\
& \text { belong to } \mathcal{L} \operatorname{is}\left(\mathbb{E}^{(s+r) / \vec{r}}, \mathbb{E}^{s / \vec{r}} \times \mathbb{E}^{s+r(1-1 / q)}\right) .
\end{aligned}
$$

Then

$$
\left(\partial_{t}+\mathcal{A}, \gamma\right) \in \mathcal{L} \operatorname{is}\left(\mathfrak{F}_{q}^{(s+r) / \vec{r}}\left(V \times \mathbb{R}^{+}\right), \mathfrak{F}_{q}^{s / \vec{r}}\left(V \times \mathbb{R}^{+}\right) \times \mathfrak{F}_{q}^{s+r(1-1 / q)}(V)\right)
$$

and

$$
\left(\partial_{t}+\mathcal{A}, \gamma\right)^{-1}=\mathcal{R} \circ\left(\partial_{t}+A+B, \boldsymbol{\gamma}\right)^{-1} \circ\left(\mathcal{R}^{c} \times \mathcal{R}^{c}\right)
$$

\section{The Flat Case}

Now we assume

$$
\begin{aligned}
& \text { (i) }(M, g)=\left(\mathbb{R}^{m}, g_{m}\right) . \\
& \text { (ii) Assumption (10.1) is satisfied. } \\
& \text { (iii) } \varepsilon^{-1}+\sum_{j=0}^{r}\left\|a_{j}\right\|_{\bar{s}, \infty} \leq \bar{\kappa} .
\end{aligned}
$$

Recall from (9.4) that $V=\mathbb{R}^{m} \times E$. We also suppose that

$$
\text { - }(s, q) \text { is } r \text {-admissible }
$$

and write $X_{\eta}^{j}:=\mathfrak{F}_{q ; \eta}^{(s+j r) / \vec{r}}(\mathbb{H}, E)$ for $j=0,1$ and $Y_{\eta}:=\mathfrak{F}_{q ; \eta}^{s+r(1-1 / q)}\left(\mathbb{R}^{m}, E\right)$.

It follows from (10.1) that the constant coefficient operator $a_{r}(x) \bullet \partial^{r}$ is normally $\varepsilon$-elliptic and $\left|a_{r}(x)\right|_{\mathcal{L}^{r}} \leq\left\|a_{r}\right\|_{\bar{s}, \infty}$, uniformly with respect to $x \in \mathbb{R}^{m}$. Hence Theorem 8.1 implies

$$
\left(\partial_{t}+\eta+a_{r}(x) \cdot \partial^{r}, \gamma\right) \in \mathcal{L} \operatorname{is}\left(X^{1}, X^{0} \times Y\right)
$$

and there exists $c_{0}=c_{0}(\bar{\kappa})$ such that, for $x \in \mathbb{R}^{m}$ and $\eta>0$,

$$
\left\|\left(\partial_{t}+\eta+a_{r}(x) \cdot \partial^{r}, \gamma\right)^{-1}\right\|_{\mathcal{L} \operatorname{is}\left(X_{\eta}^{0} \times Y_{\eta}, X_{\eta}^{1}\right)} \leq c_{0} .
$$

Since $a_{r} \in b u c^{\bar{s}}\left(\mathbb{R}^{m}, \mathcal{L}^{r}\right)$,

$$
\left|a_{r}(\delta(x+z))-a_{r}(\delta z)\right|_{\mathcal{L}^{r}} \leq c \delta^{\bar{s}}, \quad x \in Q^{m}, \quad z \in \mathbb{Z}^{m},
$$


and

$$
\sup _{x, y \in Q^{m}} \frac{\left|a_{r}(\delta(x+z))-a_{r}(\delta(y+z))\right|_{\mathcal{L}^{r}}}{|\delta(x-y)|^{\bar{s}}} \rightarrow 0 \quad \text { as } \delta \rightarrow 0,
$$

uniformly with respect to $z \in \mathbb{Z}^{m}$. With the radial retraction $h_{\delta}$ we put

$$
\mathrm{a}_{z, \delta}(x):=a_{r}\left(\delta z+h_{\delta}(x-\delta z)\right), \quad x \in \mathbb{R}^{m}, \quad z \in \mathbb{Z}^{m} .
$$

Then, as in (10.7),

$$
\left(\mathrm{a}_{z, \delta}\right)_{z \in \mathbb{Z}^{m}} \in \ell_{\infty, \text { unif }}\left(b u c^{\bar{s}}\left(\mathbb{R}^{m}, \mathcal{L}^{r}\right)\right)
$$

(where we now employ the index set $\mathbb{Z}$ ), and

$$
\mathrm{a}_{z, \delta}(x)=a_{r}(x), \quad x \in \delta\left(z+Q^{m}\right) .
$$

From this, (12.4), (12.5), and Theorems 3.1(ii) and 3.3 we infer that

$$
\left\|\left(\mathrm{a}_{z, \delta}-a_{r}(\delta z)\right) \cdot \partial^{r}\right\|_{\mathcal{L}\left(X_{\eta}^{1}, X_{\eta}^{0}\right)} \leq c\left\|a_{r}(\delta(z+\cdot))-a_{r}(\delta z)\right\|_{B C^{\bar{s}}\left(Q^{m}, \mathcal{L}^{r}\right)} \rightarrow 0
$$

as $\delta \rightarrow 0$, uniformly with respect to $z \in \mathbb{Z}^{m}$. Hence we can fix $\delta=\delta(\bar{\kappa}) \in(0,1)$ such that

$$
\left\|\left(\mathrm{a}_{z, \delta}-a(\delta z) \cdot \partial^{r}\right)\right\|_{\mathcal{L}\left(X_{\eta}^{1}, X_{\eta}^{0}\right)} \leq 1 / 2 c_{0},
$$

uniformly with respect to $\eta>0$ and $z \in \mathbb{Z}^{m}$.

We set $\kappa_{z}(x):=-z+x / \delta$ for $x \in U_{\kappa_{z}}:=\delta\left(z+Q^{m}\right)$ and $z \in \mathbb{Z}^{m}$. Then $\mathfrak{K}:=\left\{\kappa_{z} ; z \in \mathbb{Z}^{m}\right\}$ is a uniformly regular atlas for $(M, g)$. We fix a localization system $\left\{\left(\pi_{\kappa}, \chi_{\kappa}\right) ; \kappa \in \mathfrak{K}\right\}$ subordinate to $\mathfrak{K}$ and put

$$
R \boldsymbol{u}:=\sum_{\kappa} \pi_{\kappa} u_{\kappa}, \quad R^{c} u:=\left(\pi_{\kappa} u\right)
$$

for $\boldsymbol{u}=\left(u_{\kappa}\right) \in \mathfrak{F}_{q}^{s / \vec{r}}$ and $u \in \mathfrak{F}_{q}^{s / \vec{r}}\left(V \times \mathbb{R}^{+}\right)$.

The following lemma is a parameter-dependent equivalent of Theorem 9.1 . Its proof, however, is much simpler since the atlas $\mathfrak{K}$ is not explicitly involved.

Lemma 12.1. $\left(R, R^{c}\right)$ is an $\eta$-uniform r-e pair for $\left(\mathbb{E}_{q ; \eta}^{s / \vec{r}}, \mathfrak{F}_{q ; \eta}^{s / \vec{r}}\left(V \times \mathbb{R}^{+}\right)\right)$.

For easy reference we include the following well-known perturbation theorem.

Lemma 12.2. Let $X$ and $Y$ be Banach spaces and $a \in \mathcal{L}$ is $(X, Y)$. Suppose $b \in \mathcal{L}(X, Y)$ satisfies $\left\|b a^{-1}\right\| \leq 1 / 2$, then $a+b$ belongs to $\mathcal{L} \operatorname{is}(X, Y)$ and $\left\|(a+b)^{-1}\right\| \leq 2\left\|a^{-1}\right\|$.

Proof. A Neumann series argument shows that

$$
1+b a^{-1} \in \mathcal{L} \operatorname{aut}(Y) \text { and }\left\|\left(1+b a^{-1}\right)^{-1}\right\| \leq 2 .
$$

Hence the claim follows from $a+b=\left(1+b a^{-1}\right) a$.

We set

$$
\mathrm{A}_{\kappa}:=a_{z, \delta} \cdot \partial^{r} \text { for } \kappa=\kappa_{z} \in \mathfrak{K}
$$

and

$$
\mathbb{X}_{\eta}^{j}:=\mathbb{E}_{q ; \eta}^{(s+j r) / \vec{r}} \text { for } j=0,1, \quad \mathbb{Y}_{\eta}:=\mathbb{E}_{q ; \eta}^{s+r(1-1 / q)} .
$$

Clearly, $\mathbb{X}_{\eta}^{j}$ is obtained by replacing $X^{j}$ in (11.1) by $X_{\eta}^{j}$, etc. 
Lemma 12.3. Set $\mathrm{A}:=\operatorname{diag}\left[\mathrm{A}_{\kappa}\right]$. Then $\left(\boldsymbol{\partial}_{t}+\eta+\mathrm{A}, \boldsymbol{\gamma}\right) \in \mathcal{L} \operatorname{is}\left(\mathbb{X}^{1}, \mathbb{X}^{0} \times \mathbb{Y}\right)$ and

$$
\left\|\left(\boldsymbol{\partial}_{t}+\eta+\mathrm{A}, \boldsymbol{\gamma}\right)^{-1}\right\|_{\mathcal{L}\left(\mathbb{X}_{\eta}^{0} \times \mathbb{Y}_{\eta}, \mathbb{X}_{\eta}^{1}\right)} \leq c(\bar{\kappa}) \quad \eta \text {-uniformly }
$$

Proof. We put $\mathrm{A}_{\kappa}^{0}:=a(\delta z) \cdot \partial^{r}$ for $\kappa=\kappa_{z}$. Then (12.2) and (12.3) imply that $\left(\partial_{t}+\eta+\mathcal{A}_{\kappa}^{0}, \gamma_{\kappa}\right)$ is an isomorphism from $X^{1}$ onto $X^{0} \times Y$, and

$$
\left\|\left(\partial_{t}+\eta+\mathrm{A}_{\kappa}^{0}, \gamma_{\kappa}\right)^{-1}\right\|_{\mathcal{L}\left(\mathbb{X}_{\eta}^{0} \times \mathbb{Y}_{\eta}, \mathbb{X}_{\eta}^{1}\right)} \leq c_{0}, \quad \kappa \in \mathfrak{K}, \quad \eta>0 .
$$

Set $\mathrm{B}_{\kappa}:=\mathrm{A}_{\kappa}-\mathrm{A}_{\kappa}^{0} \in \mathcal{L}\left(X^{1}, X^{0}\right)$. Then $\left\|\mathrm{B}_{\kappa}\right\|_{\mathcal{L}\left(\mathbb{X}_{\eta}^{1}, \mathbb{X}_{\eta}^{0}\right)} \leq 1 / 2 c_{0}$ by (12.7), uniformly with respect to $\kappa \in \mathfrak{K}$ and $\eta>0$. Hence it follows from

$$
\left(\partial_{t}+\eta+\mathrm{A}_{\kappa}, \gamma_{\kappa}\right)=\left(\partial_{t}+\eta+\mathrm{A}_{\kappa}^{0}, \gamma_{\kappa}\right)+\left(\mathrm{B}_{\kappa}, 0\right)
$$

estimate (12.9), and Lemma 12.2 that $\left(\partial_{t}+\eta+\mathrm{A}_{\kappa}, \gamma_{\kappa}\right) \in \mathcal{L}$ is $\left(X^{1}, X^{0} \times Y\right)$ and

$$
\left\|\left(\partial_{t}+\eta+\mathrm{A}_{\kappa}, \gamma_{\kappa}\right)^{-1}\right\|_{\mathcal{L}\left(X_{\eta}^{0} \times Y_{\eta}, X_{\eta}^{1}\right)} \leq 2 c_{0},
$$

uniformly with respect to $\kappa \in \mathfrak{K}$ and $\eta>0$. Now, taking (12.6) into consideration, the assertion is clear.

The next lemma is an analogue to 11.2 in the present setting. Its proof is obtained by simplifying the demonstration of Lemma 10.1 based on the fact that the local charts do not occur in (12.8).

We set $\mathbb{W}_{\eta}:=\ell_{q}\left(\mathfrak{F}_{q ; \eta}^{(s+r-1) / \vec{r}}\right)$ if $q<\infty$, and $\mathbb{W}_{\eta}:=\ell_{\infty, \text { unif }}\left(\mathfrak{F}_{\infty ; \eta}^{(s+r-1) / \vec{r}}\right)$ if $q=\infty$.

Lemma 12.4. There exist $\mathrm{B}, \mathrm{B}^{\prime} \in \mathcal{L}\left(\mathbb{W}_{\eta}, \mathbb{X}_{\eta}^{0}\right)$ such that

$$
\begin{aligned}
\left(\partial_{t}+\mathcal{A}_{\eta}\right) \circ R & =R \circ\left(\partial_{t}+\eta+\mathrm{A}+\mathrm{B}\right), \\
R^{c} \circ\left(\partial_{t}+\mathcal{A}_{\eta}\right) & =\left(\partial_{t}+\eta+\mathrm{A}+\mathrm{B}^{\prime}\right) \circ R^{c} .
\end{aligned}
$$

Now we are ready to prove the main result of this section. Observe that $V \times J \widehat{=} \mathbb{H} \times E$.

Theorem 12.5. Let (12.1) be satisfied. There exists $\eta_{0}=\eta_{0}(\bar{\kappa}) \geq 1$ such that $\left(\partial_{t}+\mathcal{A}_{\eta}, \gamma\right) \in \mathcal{L}\left(X^{1}, X^{0} \times Y\right)$ and

$$
\left\|\left(\partial_{t}+\mathcal{A}_{\eta}, \gamma\right)^{-1}\right\|_{\mathcal{L}\left(X_{\eta}^{0} \times Y_{\eta}, X_{\eta}^{1}\right)} \leq c(\bar{\kappa}), \quad \eta \geq \eta_{0}
$$

Proof. Theorem 3.1(i) guarantees $\mathfrak{F}_{q}^{(s+r) / \vec{r}} \hookrightarrow \mathfrak{F}_{q}^{(s+r-1) / \vec{r}}$ and

$$
\|\cdot\|_{(s+r+1) / \vec{r}, q ; \eta} \leq c \eta^{-1}\|\cdot\|_{(s+r) / \vec{r}, q ; \eta}, \quad \eta>0,
$$

This implies $\mathbb{X}_{\eta}^{1} \hookrightarrow \mathbb{W}$ and $\|\cdot\|_{\mathbb{W}_{\eta}} \leq c \eta^{-1}\|\cdot\|_{\mathbb{X}_{\eta}^{1}}$ for $\eta>0$.

We write $c_{0}$ for the constant $c(\bar{\kappa})$ of Lemma 12.3. Then we get

$$
\left\|\left(\boldsymbol{\partial}_{t}+\eta+\mathrm{A}, \boldsymbol{\gamma}\right)^{-1}\right\|_{\mathcal{L}\left(\mathbb{X}_{\eta}^{0} \times \mathbb{Y}_{\eta}, \mathbb{W}_{\eta}\right)} \leq c_{0} / \eta, \quad \eta>0 .
$$

Lemma 12.4 guarantees the existence of $c_{1} \geq 1$ such that

$$
\|\mathrm{B}\|_{\mathcal{L}\left(\mathbb{W}_{\eta}, \mathbb{X}_{\eta}^{0}\right)}+\left\|\mathrm{B}^{\prime}\right\|_{\mathcal{L}\left(\mathbb{W}_{\eta}, \mathbb{X}_{\eta}^{0}\right)} \leq c_{1} \quad \eta \text {-uniformly }
$$


Hence, setting $\eta_{0}:=2 c_{1} c_{0}^{2}$, we find

$$
\left\|\left(\mathrm{B} \circ\left(\boldsymbol{\partial}_{t}+\eta+\mathrm{A}, \boldsymbol{\gamma}\right)^{-1}, 0\right)\right\|_{\mathcal{L}\left(\mathbb{X}_{\eta}^{0} \times \mathbb{Y}_{\eta}\right)} \leq 1 / 2 c_{0}, \quad \eta \geq \eta_{0} .
$$

From this and Lemma 12.2 we obtain that $\left(\boldsymbol{\partial}_{t}+\eta+\mathrm{A}+\mathrm{B}, \boldsymbol{\gamma}\right)$ belongs to $\mathcal{L}$ is $\left(\mathbb{X}^{1}, \mathbb{X}^{0} \times \mathbb{Y}\right)$ and

$$
\left\|\left(\boldsymbol{\partial}_{t}+\eta+\mathrm{A}+\mathrm{B}, \gamma\right)^{-1}\right\|_{\mathcal{L}\left(\mathbb{X}_{\eta}^{0} \times \mathbb{Y}_{\eta}, \mathbb{X}_{\eta}^{1}\right)} \leq 2 c_{0}, \quad \eta \geq \eta_{0} .
$$

The same argument shows that (12.11) holds with B replaced by $\mathrm{B}^{\prime}$.

It is obvious that $\gamma \circ R=R \circ \boldsymbol{\gamma}$ and $R^{c} \circ \boldsymbol{\gamma}=\gamma \circ R^{c}$. Using this and (12.10), the assertion thus follows from Lemma 12.1 and (the analogue of) Corollary 11.3 .

Now we assume that $(s, q)$ is 1-admissible. Going through the above proofs, neglecting any reference to $t \in \mathbb{R}^{+}$, using Theorem 7.1 instead of Theorem 8.1 , and appealing to Corollary 10.2 instead of Corollary 11.3, etc., we obtain the following resolvent estimate. Details are left to the reader.

Theorem 12.6. Let assumption (12.1) be satisfied, but assume that $(s, q)$ is 1-admissible. Then there exist $\eta_{0}=\eta_{0}(\bar{\kappa}) \geq 1$ such that $\lambda+\mathcal{A}_{\eta}$ belongs to $\mathcal{L} \operatorname{is}\left(\mathfrak{F}_{q}^{s+r}, \mathfrak{F}_{q}^{s}\right)$ and

$$
(|\lambda|+\eta)^{1-j}\left\|\left(\lambda+\mathcal{A}_{\eta}\right)^{-1}\right\|_{\mathcal{L}\left(\mathfrak{F}_{q ; \eta}^{s}, \mathfrak{F}_{q ; \eta}^{s+j+r}\right)} \leq c(\kappa)
$$

for $\operatorname{Re} \lambda \geq 0$ and $\eta>0$.

\section{Proof of the Main Theorems}

After all the preparation in the preceding sections it is no longer too difficult to demonstrate the validity of Theorems 1.23 and 1.30 .

Proof of Theorem 1.23thm.1.23. First we observe that the assumptions on $(s, q)$, where $q:=\infty$ in claim (ii), amount to: $(s, q)$ is $r$-admissible. We fix $\bar{\kappa}$ satisfying

$$
\varepsilon^{-1}+\sum_{j=0}^{r}\left\|a_{j}\right\|_{\bar{s} / \vec{r}, \infty} \leq \bar{\kappa} .
$$

(1) Assume $0<\bar{s}<1$ and $\mathcal{A}$ is independent of $t \in \mathbb{R}^{+}$. Define $a_{\kappa}$ by (10.5). It follows from (10.7), (10.8), and Theorem 12.5 that there are $\eta_{0}=\eta_{0}(\bar{\kappa}) \geq 1$ and $c_{0}=c_{0}(\bar{\kappa}) \geq 1$ such that

$$
\left(\partial_{t}+\eta+a_{\kappa} \cdot \partial^{r}, \gamma_{\kappa}\right) \in \mathcal{L} \operatorname{is}\left(X^{1}, X^{0} \times Y\right)
$$

and

$$
\left\|\left(\partial_{t}+\eta+a_{\kappa} \cdot \partial^{r}, \gamma_{\kappa}\right)^{-1}\right\|_{\mathcal{L}\left(X_{\eta}^{0} \times Y_{\eta}, X_{\eta}^{1}\right)} \leq c_{0},
$$

uniformly with respect to $\eta \geq \eta_{0}$ and $\kappa \in \mathfrak{K}$. From this, (10.9), and Theorem 3.2 we infer $\left(\boldsymbol{\partial}_{t}+\eta+A, \boldsymbol{\gamma}\right) \in \mathcal{L}$ is $\left(\mathbb{X}^{1}, \mathbb{X}^{0} \times \mathbb{Y}\right)$ and

$$
\left\|\left(\boldsymbol{\partial}_{t}+\eta+A, \boldsymbol{\gamma}\right)^{-1}\right\|_{\mathcal{L}\left(\mathbb{X}_{\eta}^{0} \times \mathbb{Y}_{\eta}, \mathbb{X}_{\eta}^{1}\right)} \leq c_{0}, \quad \eta \geq \eta_{0}
$$


Using (10.10) and the arguments of the proof of Theorem 12.5 we see that we can find $\eta \geq \eta_{0} \geq 1$ so that

$$
\left(\boldsymbol{\partial}_{t}+\eta+A+B, \boldsymbol{\gamma}\right),\left(\boldsymbol{\partial}_{t}+\eta+A+B^{\prime}, \boldsymbol{\gamma}\right) \in \mathcal{L} \operatorname{is}\left(\mathbb{X}^{1}, \mathbb{X}^{0} \times \mathbb{Y}\right)
$$

and the inverses of these linear operators are bounded by $c(\bar{\kappa})$.

Set $\mathcal{X}^{j}:=\mathfrak{F}_{q}^{(s+j r) / \vec{r}}\left(V \times \mathbb{R}^{+}\right)$for $j=0,1$, and $\mathcal{Y}:=\mathfrak{F}_{q}^{s+r(1-1 / q)}(V)$. Then Corollary 11.3 implies

$$
\left(\partial_{t}+\mathcal{A}_{\eta}, \gamma\right) \in \mathcal{L} \operatorname{is}\left(\mathcal{X}^{1}, \mathcal{X}^{0} \times \mathcal{Y}\right), \quad\left\|\left(\partial_{t}+\mathcal{A}_{\eta}, \gamma\right)^{-1}\right\|_{\mathcal{L}\left(\mathcal{X}^{0} \times \mathcal{Y}, \mathcal{X}^{1}\right)} \leq c(\tilde{\kappa}) .
$$

(2) Suppose $0<\bar{s}<1$. We write $\mathcal{X}^{j}(S):=\mathfrak{F}_{q}^{(s+j r) / \vec{r}}(V \times[0, S])$ for $S>0$. Given $\tau \in J=J_{T}$, we denote by $\partial_{t}+\mathcal{A}(\tau)$ the autonomous operator whose coefficients are frozen at $t=\tau$. Then $\mathcal{A}(\tau)$ is $\bar{s}$-regular and normally $\varepsilon$-elliptic, uniformly with respect to $\tau \in J$. Thus, by step (1), $\left(\partial_{t}+\mathcal{A}_{\eta}(\tau), \gamma\right)$ belongs to $\mathcal{L}$ is $\left(\mathcal{X}^{1}, \mathcal{X}^{0} \times \mathcal{Y}\right)$ and

$$
\left\|\left(\partial_{t}+\mathcal{A}_{\eta}(\tau), \gamma\right)^{-1}\right\|_{\mathcal{L}\left(\mathcal{X}^{0} \times \mathcal{Y}, \mathcal{X}^{1}\right)} \leq c(\bar{\kappa}), \quad 0 \leq \tau \leq T .
$$

The fact that the coefficients of $\mathcal{A}$ belong to $b c^{\bar{s}} / \vec{r}(V \times J)$ implies (similarly as in Section 12)

$$
\|\mathcal{A}(\tau+\cdot)-\mathcal{A}(\tau)\|_{\mathcal{L}\left(\mathcal{X}^{1}(S), \mathcal{X}^{0}(S)\right)} \rightarrow 0 \quad \text { as } S \rightarrow 0,
$$

uniformly with respect to $\tau \in J$. Since

$$
\left(\partial_{t}+\mathcal{A}_{\eta}, \gamma\right)=\left(\partial_{t}+\mathcal{A}_{\eta}(\tau), \gamma\right)+(\mathcal{A}(\tau+\cdot)-\mathcal{A}(\tau), 0) \text { on } M \times[\tau, T],
$$

we infer from (13.1), (13.2), and Lemma 12.2 that there exist $S \in(0, T)$ and $k \in \dot{\mathbb{N}}$ such that

$$
\left(\partial_{t}+\mathcal{A}_{\eta}(j S+\cdot)\right) v=f(j S+\cdot), \quad \gamma v=w
$$

has for each $w \in \mathcal{Y}$ a unique solution $V_{j}(w) \in \mathcal{X}^{1}(S)$ if $0 \leq j \leq k-1$, and a unique solution $V_{k}(w) \in \mathcal{X}^{1}(\min \{S, T-k S\})$. We set $v_{0}:=V_{0}\left(u_{0}\right)$ and $v_{i}:=V_{i}\left(v_{i-1}(S)\right)$ for $1 \leq i \leq k$. For $t=i S+s$ we define $u$ by $u(t):=v_{i}(s)$, where $0 \leq i<k$ and $0 \leq s \leq \min \{S, T-i S\}$. The trace theorem shows that $u$ belongs to $\mathfrak{F}_{q}^{(s+r) / \vec{r}}(V \times J)$ and is the unique solution of $\left(\partial_{t}+\mathcal{A}_{\eta}\right) u=f$ on $V \times J$ satisfying $\gamma u=u_{0}$.

(3) Let $0<\bar{s}<1$. Set $f^{\eta}:=e^{t \eta} f$. Then $u \in \mathcal{X}^{1}(T)$ satisfies $\left(\partial_{t}+\mathcal{A}\right) u=f^{\eta}$ and $\gamma u=u_{0}$ iff $u=e^{t \eta} v$ and $v \in \mathcal{X}^{1}(T)$ conforms to $\left(\partial_{t}+\mathcal{A}_{\eta}\right) v=f$ and $\gamma v=u_{0}$. Since $f \mapsto f^{\eta}$ is an automorphism of $\mathcal{X}^{0}(T)$, we see from the preceding step that the theorem holds under the present additional hypothesis.

(4) We put $\mathcal{Y}^{s}(V):=\mathfrak{F}_{q}^{s / \vec{r}}(V \times J)$ and $\mathcal{Z}^{s}(V):=\mathfrak{F}_{q}^{s+r(1-1 / q)}(V)$. Suppose

$$
r \leq s<\bar{s}<r+1
$$

and set $s_{0}:=s-r$. Let $\left(f, u_{0}\right)$ belong to $\mathcal{Y}^{s}(V) \times \mathcal{Z}^{s}(V)$. Since

$$
\mathcal{Y}^{s}(V) \times \mathcal{Z}^{s}(V) \hookrightarrow \mathcal{Y}^{s_{0}}(V) \times \mathcal{Z}^{s_{0}}(V),
$$


it follows from what we have already shown that there exists a unique $u \in \mathcal{Y}^{s_{0}+r}(V)=\mathcal{Y}^{s}(V)$ satisfying $\left(\partial_{t}+\mathcal{A}\right) u=f$ on $V \times J$ and $\gamma u=u_{0}$. Let $1 \leq i \leq r$. By applying $\nabla^{i}$ we get

$$
\left(\partial_{t}+\mathcal{A}\right) \nabla^{i} u=\mathcal{A}_{i} u+\nabla^{i} f \text { on } V_{\tau+i}^{\sigma} \times J, \quad \gamma \nabla^{i} u=\nabla^{i} u_{0},
$$

where

$$
\mathcal{A}_{i} u:=-\sum_{j=0}^{r} \sum_{k=1}^{i}\left(\begin{array}{l}
i \\
k
\end{array}\right) \nabla^{k} a_{j} \cdot \nabla^{i-k} u .
$$

Note

$$
\nabla^{i-k} u \in \mathcal{Y}^{s-i+k}\left(V_{\tau+i-k}^{\sigma}\right) \hookrightarrow \mathcal{Y}^{s_{0}}\left(V_{\tau+i-k}^{\sigma}\right)
$$

and

$$
\nabla^{k} a_{j} \in b c^{(\bar{s}-k) / \vec{r}}\left(V_{\tau+\sigma+k}^{\sigma+\tau+j}(\mathcal{L}(F))\right) \hookrightarrow b c^{(\bar{s}-r) / \vec{r}}\left(V_{\tau+\sigma+k}^{\sigma+\tau+j}(\mathcal{L}(F))\right) .
$$

From this it follows, due to $\bar{s}-k \geq s_{0}$ with $\bar{s}-k>s_{0}$ if $q<\infty$, that

$$
\mathcal{A}_{j} u+\nabla^{i} f \in \mathcal{Y}^{s_{0}}\left(V_{\tau+i}^{\sigma}\right), \quad \nabla^{i} u_{0} \in \mathcal{Z}^{s_{0}}\left(V_{\tau+i}^{\sigma}\right) .
$$

Hence the results of the preceding step guarantee that

$$
\nabla^{i} u=\left(\partial_{t}+\mathcal{A}, \gamma\right)^{-1}\left(\mathcal{A}_{i} u+\nabla^{i} f, \nabla^{i} u_{0}\right) \in \mathcal{Y}^{s}\left(V_{\tau+i}^{\sigma}\right), \quad 1 \leq i \leq r .
$$

Analogously,

$$
\left(\partial_{t}+\mathcal{A}\right) \partial_{t} u=\mathcal{A}_{0} u+\partial_{t} f \text { on } V \times J, \quad \gamma \partial_{t} u=-\mathcal{A}(0) u_{0}+f(0),
$$

where

$$
\mathcal{A}_{0} u:=-\sum_{j=0}^{r} \partial_{t} a_{j} \cdot \nabla^{j} u \in \mathcal{Y}^{s_{0}}(V)
$$

due to $\partial_{t} a_{j} \in b c^{(\bar{s}-r) / \vec{r}}\left(V_{\tau+\sigma}^{\sigma+\tau+j}(\mathcal{L}(F))\right)$. It also follows from the trace theorem that

$$
-\mathcal{A}(0) u_{0}+f(0) \in \mathcal{Z}^{s_{0}}(V)
$$

Now we infer from (13.5), (13.6), and the results of step (3) that

$$
\partial_{t} u=\left(\partial_{t}+\mathcal{A}, \gamma\right)^{-1}\left(\mathcal{A}_{0} u+\partial_{t} f,-\mathcal{A}(0) u_{0}+f(0)\right) \in \mathcal{Y}^{s}(V) .
$$

It follows from (13.4), (13.7), and (1.16), (1.17) that $u \in \mathcal{Y}^{s+r}(V)$. It is not difficult to check that the map $\left(f, u^{0}\right) \mapsto u$ is continuous from $\mathcal{Y}^{s}(V) \times \mathcal{Z}^{s}$ onto $\mathcal{Y}^{s+r}(V)$. This proves the theorem if (13.3) is satisfied.

(5) Assume $r<\bar{s}<r+1$ and $1<s<r$ with $s \notin \mathbb{N}$. Choose $s_{0} \in(0,1)$ and $s_{1} \in(r, \bar{s})$. Then it follows from steps (3) and (4) that

$$
\left(\partial_{t}+\mathcal{A}, \gamma\right) \in \mathcal{L} \operatorname{is}\left(\mathfrak{F}_{q}^{\left(s_{j}+r\right) / \vec{r}}(V \times J), \mathfrak{F}_{q}^{s_{j} / \vec{r}}(V \times J) \times \mathfrak{F}_{q}^{s_{j}+r(1-1 / q)}(V)\right)
$$

for $j=0,1$. In [11] it is shown that, setting $\theta:=\left(s-s_{0}\right) /\left(s_{1}-s_{0}\right)$,

$$
\left(\mathfrak{F}_{q}^{\left(s_{0}+r\right) / \vec{r}}(V \times J), \mathfrak{F}_{q}^{\left(s_{1}+r\right) / \vec{r}}(V \times J)\right)_{\theta, q}^{0} \doteq \mathfrak{F}_{q}^{(s+r) / \vec{r}}(V \times J)
$$

and

$$
\left(\mathfrak{F}_{q}^{s_{0}+r(1-1 / q)}(V), \mathfrak{F}_{q}^{s_{1}+r(1-1 / q)}(V)\right)_{\theta, q}^{0} \doteq \mathfrak{F}_{q}^{s+r(1-1 /)}(V) .
$$


Thus we get the assertion in the present case from 13.8 by interpolation. This proves the claim for $0 \leq s \leq \bar{s}$ with $r<\bar{s}<r+1$, provided $(s, q)$ is $r$-admissible. The general case follows now by induction.

Proof of Theorem 1.30thm.1.30. We modify the preceding proof by omitting $t$ and all considerations with reference to it and invoke Theorem 12.6 instead of 12.5. As for the analogue to step (4), we use the fact that $u \in \mathfrak{F}_{q}^{s+1}(V)$ iff $u \in \mathfrak{F}_{q}^{s}(V)$ and $\nabla u \in \mathfrak{F}_{q}^{s}\left(V_{\tau+1}^{\sigma}\right)$. Hence interpolation is not needed here. Then we get the existence of $\eta \geq 1$ such that $\lambda+\mathcal{A} \in \mathcal{L} \operatorname{is}\left(\mathfrak{F}_{q}^{s+r}(V), \mathfrak{F}_{q}^{s}(V)\right)$ and

$$
\left\|(\lambda+\mathcal{A})^{-1}\right\|_{\mathcal{L}\left(\mathfrak{F}_{q}^{s}(V), \mathfrak{F}_{q}^{s+j r}(V)\right)} \leq c /(1+|\lambda|)^{1-j}
$$

for $\operatorname{Re} \lambda \geq \eta$ and $j=0,1$. This proves the claim due to the density of $\mathfrak{F}_{q}^{s+r}(V)$ in $\mathfrak{F}_{q}^{s}(V)$.

\section{References}

[1] M.S. Agranovich, M.I. Vishik. Elliptic problems with a parameter and parabolic problems of general type. Russ. Math. Surveys, 19 (1964), 53-157.

[2] H. Amann. Ordinary Differential Equations. W. de Gruyter \& Co., Berlin, 1990.

[3] H. Amann. Linear and Quasilinear Parabolic Problems, Volume I: Abstract Linear Theory. Birkhäuser, Basel, 1995.

[4] H. Amann. Operator-valued Fourier multipliers, vector-valued Besov spaces, and applications. Math. Nachr., 186 (1997), 5-56.

[5] H. Amann. Elliptic operators with infinite-dimensional state spaces. J. Evol. Equ., 1 (2001), 143-188.

[6] H. Amann. Anisotropic Function Spaces and Maximal Regularity for Parabolic Problems. Part 1: Function Spaces. Jindřich Nečas Center for Mathematical Modeling, Lecture Notes, 6, Prague, 2009.

[7] H. Amann. Anisotropic function spaces on singular manifolds, 2012. arXiv:1204.0606.

[8] H. Amann. Function spaces on singular manifolds. Math. Nachr., 286 (2012), $436-475$.

[9] H. Amann. Uniformly regular and singular Riemannian manifolds. In Elliptic and parabolic equations, volume 119 of Springer Proc. Math. Stat., pages 1-43. Springer, Cham, 2015.

[10] H. Amann. Parabolic equations on uniformly regular Riemannian manifolds and degenerate initial boundary value problems. In Recent Developments of Mathematical Fluid Mechanics, H. Amann, Y. Giga, H. Kozono, H. Okamoto, M. Yamazaki (Eds.), pages 43-77. Birkhäuser, Basel, 2016.

[11] H. Amann. Linear and Quasilinear Parabolic Problems, Volume II: Function Spaces and Linear Differential Operators. 2017. In preparation.

[12] H. Amann, M. Hieber, G. Simonett. Bounded $H_{\infty}$-calculus for elliptic operators. Diff. Int. Equ., 7 (1994), 613-653.

[13] S.B. Angenent. Nonlinear analytic semiflows. Proc. Royal Soc. Edinburgh, 115A (1990), 91-107. 
[14] G. Da Prato, P. Grisvard. Sommes d'opérateurs linéaires et équations différentielles opérationelles. J. Math. Pures Appl., 54 (1975), 305-387.

[15] E.B. Davies. Heat Kernels and Spectral Theory. Cambridge Univ. Press, Cambridge, 1989.

[16] S.P. Degtyarev. On Fourier multipliers in function spaces with partial Hölder condition and their application to the linearized Cahn-Hilliard equation with dynamic boundary conditions. volume 4 of Evolution Equations and Control Theory, pages 391-429. 2015.

[17] R. Denk, M. Hieber, J. Prüss. $\mathcal{R}$-boundedness, Fourier multipliers and problems of elliptic and parabolic type. Mem. Amer. Math. Soc., 166(788) (2003).

[18] R. Denk, T. Seger. Inhomogeneous boundary value problems in spaces of higher regularity. In Recent Developments of Mathematical Fluid Mechanics, H. Amann, Y. Giga, H. Kozono, H. Okamoto, M. Yamazaki (Eds.), pages 157173. Birkhäuser, Basel, 2016.

[19] J. Dieudonné. Eléments d'Analyse III. Gauthier-Villars, Paris, 1974.

[20] M. Disconzi, Y. Shao, G. Simonett. Remarks on uniformly regular Riemannian manifolds. Math. Nachr., 289 (2016), 232-242.

[21] G. Dore. $L^{p}$ regularity for abstract differential equations. In Functional analysis and related topics, 1991 (Kyoto), Lecture Notes in Math., 1540, pages 25-38. Springer-Verlag, Berlin, 1993.

[22] G. Dore, A. Venni. On the closedness of the sum of two closed operators. Math. Z., 196 (1987), 189-201.

[23] S.D. Eidelman, N.V. Zhitarashu. Parabolic Boundary Value Problems. Birkhäuser Verlag, Basel, 1998.

[24] J. Escher, J. Prüss, G. Simonett. Analytic solutions for a Stefan problem with Gibbs-Thomson correction. J. Reine Angew. Math., 563 (2003), 1-52.

[25] A. Friedman. Partial differential equations of parabolic type. Prentice-Hall, Inc., Englewood Cliffs, N.J., 1964.

[26] A. Grigor'yan. Heat Kernel and Analysis on Manifolds. Amer. Math. Soc., Providence, RI, 2009.

[27] G. Grubb. Parameter-elliptic and parabolic pseudodifferential boundary problems in global $L_{p}$ Sobolev spaces. Math. Z., 218 (1995), 43-90.

[28] G. Grubb. Functional Calculus of Pseudodifferential Boundary Problems. Birkhäuser, Boston, MA, 1996.

[29] G. Grubb, N.J. Kokholm. A global calculus of parameter-dependent pseudodifferential boundary problems in $L_{p}$ Sobolev spaces. Acta Math., 171 (1993), $165-229$.

[30] M. Hieber, J. Prüss. Heat kernels and maximal $L^{p}-L^{q}$ estimates for parabolic evolution equations. Comm. Partial Differential Equations, 22(9-10) (1997), 1647-1669.

[31] L. Hörmander. The Analysis of Linear Partial Differential Operators I. Springer Verlag, Berlin, 1983.

[32] T. Kato. Perturbation theory for linear operators. Springer, Berlin, 1995.

[33] O.A. Ladyzhenskaya. A multiplier theorem in nonhomogeneous Hölder spaces and some of its applications. J. Math. Sci. (N.Y.), 115 (2003), 2792-2802. 
[34] O.A. Ladyzhenskaya, V.A. Solonnikov, N.N. Ural'ceva. Linear and Quasilinear Equations of Parabolic Type. Amer. Math. Soc., Transl. Math. Monographs, Providence, R.I., 1968.

[35] J. LeCrone, G. Simonett. On the flow of non-axisymmetric perturbations of cylinders via surface diffusion. 260 (2016), 5510-5531.

[36] A. Lunardi. Analytic Semigroups and Optimal Regularity in Parabolic Problems. Birkhäuser, Basel, 1995.

[37] A.L. Mazzucato, V. Nistor. Mapping properties of heat kernels, maximal regularity, and semi-linear parabolic equations on noncompact manifolds. J. Hyperbolic Differ. Equ., 3(4) (2006), 599-629.

[38] L.I. Nicolaescu. Lectures on the geometry of manifolds. World Scientific Publishing Co. Pte. Ltd., Hackensack, NJ, 2007.

[39] J. Prüss, Simonett G. Moving Interfaces and Quasilinear Parabolic Evolution Equations, volume 105 of Monographs in Mathematics. Birkhäuser, Basel, 2016.

[40] Y. Shao. Real analytic solutions for the Willmore flow. In Proceedings of the Ninth MSU-UAB Conference on Differential Equations and Computational Simulations, volume 20 of Electron. J. Differ. Equ. Conf., pages 151-164, 2013.

[41] Y. Shao. Continuous maximal regularity on singular manifolds and its applications, 2014. arXiv:1410.1082,

[42] Y. Shao. A family of parameter-dependent diffeomorphisms acting on function spaces over a Riemannian manifold and applications to geometric flows. NoDEA Nonlinear Differential Equations Appl., 22(1) (2015), 45-85.

[43] Y. Shao, G. Simonett. Continuous maximal regularity on uniformly regular Riemannian manifolds. J. Evol. Equ., 14(1) (2014), 211-248.

[44] V.A. Solonnikov. On boundary value problems for linear parabolic systems of differential equations of general form. Proc. Steklov Inst. Math., 83 (1965), $1-184$.

[45] V.A. Solonnikov. Maximal regularity and weak solutions of linear parabolic equations, 2015. Talk given at the conference Mathematical Fluid Mechanics: Old Problems, New Trends — a week for W. Zajạczkowski, Bedlewo, 30 August 2015 - 5 September 2015.

[46] M.E. Taylor. Partial differential equations II. Qualitative studies of linear equations, volume 116 of Applied Mathematical Sciences. Springer, New York, 2011.

[47] H. Triebel. Interpolation Theory, Function Spaces, Differential Operators. North Holland, Amsterdam, 1978.

[48] H. Triebel. Theory of Function Spaces. Birkhäuser, Basel, 1983.

Herbert Amann

Math. Institut

Universität Zürich

Winterthurerstr. 190

CH 8057 Zürich

Switzerland

e-mail: herbert.amann@math.uzh.ch 